\title{
Geometric Analysis of the Linear Boltzmann Equation I. Trend to Equilibrium
}

\author{
Daniel Han-Kwan' ${ }^{1}$ - Matthieu Léautaud ${ }^{2}$
}

Received: 21 October 2015 / Accepted: 22 October 2015 / Published online: 1 December 2015

(C) Springer International Publishing AG 2015

\begin{abstract}
This work is devoted to the analysis of the linear Boltzmann equation on the torus, in the presence of a force deriving from a potential. The collision operator is allowed to be degenerate in the following two senses: (1) the associated collision kernel may vanish in a large subset of the phase space; (2) we do not assume that it is bounded below by a Maxwellian at infinity in velocity. We study how the association of transport and collision phenomena can lead to convergence to equilibrium, using concepts and ideas from control theory. We prove two main classes of results. On the one hand, we show that convergence towards an equilibrium is equivalent to an almost everywhere geometric control condition. The equilibria (which are not necessarily Maxwellians with our general assumptions on the collision kernel) are described in terms of the equivalence classes of an appropriate equivalence relation involving transport and collisions. On the other hand, we characterize the exponential convergence to equilibrium in terms of the Lebeau constant, which involves some averages of the collision frequency along the flow of the transport. We also explain how to handle the case of linear Boltzmann equations posed on the phase space associated to a compact Riemannian manifold without boundary.
\end{abstract}

Keywords Kinetic theory - Control theory - Linear Boltzmann equation · Large time behaviour $\cdot$ Hypocoercivity $\cdot$ Geometric control conditions

\footnotetext{
$凶$ Daniel Han-Kwan

daniel.han-kwan@polytechnique.edu

Matthieu Léautaud

leautaud@math.univ-paris-diderot.fr

1 CNRS and École Polytechnique, Centre de Mathématiques Laurent Schwartz UMR7640, 91128 Palaiseau Cedex, France

2 Institut de Mathématiques de Jussieu-Paris Rive Gauche UMR7586, Batiment Sophie Germain, Université Paris Diderot, 75205 Paris Cedex 13, France
} 
Mathematics Subject Classification Primary: 35B40 $\cdot 76 \mathrm{P} 05 \cdot 82 \mathrm{C} 40 \cdot 82 \mathrm{C} 70$;

Secondary: 93C20

\section{Contents}

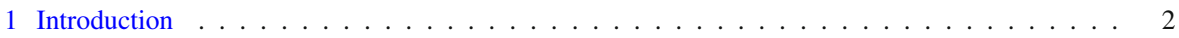

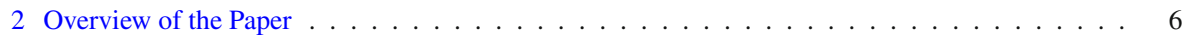

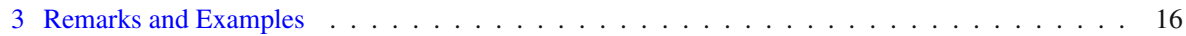

4 Preliminary Results . . . . . . . . . . . . . . . . . . . . . . . . . . . . . . . . . . 19

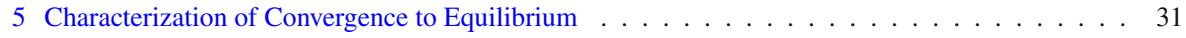

6 Application to Particular Classes of Collision Kernels . . . . . . . . . . . . . . . . . . . . 44

7 Characterization of Exponential Convergence . . . . . . . . . . . . . . . . . . . . . 49

8 Remarks on Lower Bounds for Convergence when $C^{-}(\infty)=0 \ldots \ldots \ldots \ldots$. . . . . . 58

9 The Case of a General Compact Riemannian Manifold . . . . . . . . . . . . . . . . . . . 59

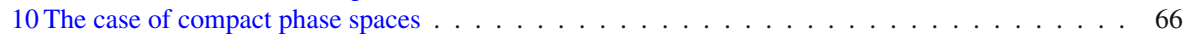

Appendix 1: A Stabilization Criterion $\ldots \ldots \ldots \ldots \ldots$. . . . . . . . . . . 67

Appendix 2: Velocity Averaging Lemmas . . . . . . . . . . . . . . . . . . . . 69

Appendix 3: Reformulation of Some Geometric Properties . . . . . . . . . . . . . . . . . 74

Appendix 4: Proof of Proposition $3.2 \ldots \ldots \ldots \ldots \ldots$. . . . . . . . . . . . . . 77

Appendix 5: Other Linear Boltzmann Type Equations . . . . . . . . . . . . . . . . . . . . 77

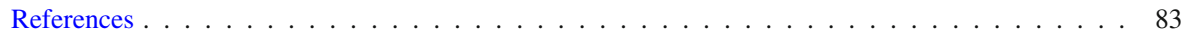

\section{Introduction}

This paper is concerned with the study of the linear Boltzmann equation

$$
\partial_{t} f+v \cdot \nabla_{x} f-\nabla_{x} V \cdot \nabla_{v} f=\int_{\mathbb{R}^{d}}\left[k\left(x, v^{\prime}, v\right) f\left(v^{\prime}\right)-k\left(x, v, v^{\prime}\right) f(v)\right] d v^{\prime}
$$

for $x \in \mathbb{T}^{d}, v \in \mathbb{R}^{d}, d \in \mathbb{N}^{*}$, where $\mathbb{T}^{d}:=\mathbb{R}^{d} / \mathbb{Z}^{d}$ is the flat torus. The linear Boltzmann equation is a classical model of statistical physics, allowing to describe the interaction between particles and a fixed background [11-13]. Among many possible applications, we mention the modeling of semi-conductors, cometary flows, or neutron transport. We refer the reader interested by further physical considerations or by a discussion of the validity of (1.1) in these contexts to [11, Chapter IV, §3] or [12, Chapter I, §5]. We also point out that this equation can be derived in various settings: see for instance [21] in the context of quantum scattering, or [9] in the context of a gas of interacting particles.

In (1.1), the unknown function $f=f(t, x, v)$ is the so-called distribution function; the quantity $f(t, x, v) d v d x$ can be understood as the (non-negative) density at time $t$ of particles whose position is close to $x$ and velocity close to $v$. The function $V$ is a potential which drives the dynamics of particles; we shall assume throughout this work that $V$ is smooth, more precisely that $V \in W^{2, \infty}\left(\mathbb{T}^{d}\right)$. We normalize the potential so that

$$
\int_{\mathbb{T}^{d}} e^{-V} d x=1
$$


This may always be assumed by changing the potential $V$ (which only appears in (1.1) through $\left.\nabla_{x} V\right)$ into $V+\log \left(\int_{\mathbb{T}^{d}} e^{-V} d x\right)$. The linear Boltzmann equation (1.1) is a typical example of a so-called hypocoercive equation, in the sense of Villani [42]. It is made of a conservative part, namely the kinetic transport operator $v \cdot \nabla_{x}-\nabla_{x} V \cdot \nabla_{v}$ associated to the hamiltonian $H(x, v)=\frac{1}{2}|v|^{2}+V(x)$, and a degenerate dissipative part which is the collision operator (i.e. the right hand-side of (1.1)). According to the hypocoercivity mechanism of [42], only the interaction between the two parts can lead to convergence to some global equilibrium.

The function $k$ is the so-called collision kernel, which describes the interaction between the particles and the background. In the following, we shall denote by $\mathscr{C}(f)$ the collision operator, which can be split as

$$
\mathscr{C}(f)(x, v)=\mathscr{C}^{+}(f)(x, v)+\mathscr{C}^{-}(f)(x, v),
$$

where

$$
\begin{aligned}
\mathscr{C}^{+}(f)(x, v) & =\int_{\mathbb{R}^{d}} k\left(x, v^{\prime}, v\right) f\left(v^{\prime}\right) d v^{\prime}, \\
\mathscr{C}^{-}(f)(x, v) & =-\left(\int_{\mathbb{R}^{d}} k\left(x, v, v^{\prime}\right) d v^{\prime}\right) f(v)
\end{aligned}
$$

are respectively the gain and the loss term. A first property of this operator is that, due to symmetry reasons, the formal identity holds:

$$
\text { for all } x \in \mathbb{T}^{d}, \quad \int_{\mathbb{R}^{d}} \int_{\mathbb{R}^{d}}\left[k\left(x, v^{\prime}, v\right) f\left(v^{\prime}\right)-k\left(x, v, v^{\prime}\right) f(v)\right] d v^{\prime} d v=0 .
$$

This, together with the fact that the vector field $v \cdot \nabla_{x}-\nabla_{x} V \cdot \nabla_{v}$ is divergence free, implies that the mass is conserved: any solution $f$ of (1.1) satisfies

$$
\text { for all } t \geq 0, \quad \frac{d}{d t} \int_{\mathbb{T}^{d} \times \mathbb{R}^{d}} f(t, x, v) d v d x=0 .
$$

We shall now list the assumptions we make on the collision kernel $k$.

A1. The collision kernel $k$ belongs to the class $C^{0}\left(\mathbb{T}^{d} \times \mathbb{R}^{d} \times \mathbb{R}^{d}\right)$ and is nonnegative. A2. Introducing the Maxwellian distribution

$$
\mathcal{M}(v):=\frac{1}{(2 \pi)^{d / 2}} e^{-\frac{|v|^{2}}{2}}
$$

we assume that $\mathcal{M}$ cancels the collision operator, that is

$$
\text { for all }(x, v) \in \mathbb{T}^{d} \times \mathbb{R}^{d}, \quad \int_{\mathbb{R}^{d}}\left[k\left(x, v^{\prime}, v\right) \mathcal{M}\left(v^{\prime}\right)-k\left(x, v, v^{\prime}\right) \mathcal{M}(v)\right] d v^{\prime}=0 \text {. }
$$


A3. Assume that

$$
x \mapsto \int_{\mathbb{R}^{d} \times \mathbb{R}^{d}} k^{2}\left(x, v^{\prime}, v\right) \frac{\mathcal{M}\left(v^{\prime}\right)}{\mathcal{M}(v)} d v^{\prime} d v \in L^{\infty}\left(\mathbb{T}^{d}\right) .
$$

It will sometimes be convenient to work with the function

$$
\tilde{k}\left(x, v^{\prime}, v\right):=\frac{k\left(x, v^{\prime}, v\right)}{\mathcal{M}(v)} .
$$

With this notation, Assumptions A2 and $\mathbf{A 3}$ may be rephrased in a more symmetric way as

$$
\begin{aligned}
\text { for all }(x, v) & \in \mathbb{T}^{d} \times \mathbb{R}^{d}, \quad \int_{\mathbb{R}^{d}} \tilde{k}\left(x, v^{\prime}, v\right) \mathcal{M}\left(v^{\prime}\right) d v^{\prime}=\int_{\mathbb{R}^{d}} \tilde{k}\left(x, v, v^{\prime}\right) \mathcal{M}\left(v^{\prime}\right) d v^{\prime} ; \\
x & \mapsto \int_{\mathbb{R}^{d} \times \mathbb{R}^{d}} \tilde{k}^{2}\left(x, v^{\prime}, v\right) \mathcal{M}\left(v^{\prime}\right) \mathcal{M}(v) d v^{\prime} d v \in L^{\infty}\left(\mathbb{T}^{d}\right) .
\end{aligned}
$$

Note that with assumption A2, the function $(x, v) \mapsto \mathcal{M}(v) e^{-V(x)}=\frac{1}{(2 \pi)^{d / 2}}$ $e^{-H(x, v)}$, which we shall call the Maxwellian equilibrium, cancels both the transport operator and the collision operator and thus is a stationary solution of (1.1).

Assumption A3 is a mild growth condition that is in particular satisfied in the standard cases where $\tilde{k}$ is bounded or has a polynomial growth in the variables $v$ and $v^{\prime}$. One of its interests is that the gain operator $\mathscr{C}^{+}$is then a bounded operator in the functional spaces under interest (see Section 4.1). In particular, this assumption will allow to prove well-posedness on appropriate weighted Lebesgue spaces and to justify the associated dissipation identity.

Assumption $\mathbf{A 3}$ is satisfied by kernels $k\left(x, v, v^{\prime}\right)=\tilde{k}\left(x, v, v^{\prime}\right) \mathcal{M}\left(v^{\prime}\right)$ for instance as soon as we have a bound of the form

$$
\tilde{k}\left(x, v, v^{\prime}\right) \leq \lambda e^{\varepsilon_{1}|v|^{2}+\varepsilon_{2}\left|v^{\prime}\right|^{2}}, \quad \text { with } \varepsilon_{1}<\frac{1}{4}, \quad \varepsilon_{2}<\frac{1}{4} .
$$

Before going any further, let us present usual classes of examples of collision kernels covered by Assumptions A1-A3 and addressed in the present article.

E1. "Symmetric" collision kernels. Let $k$ be a collision kernel verifying A1 and A3. We moreover require $\tilde{k}$ to be symmetric with respect to $v$ and $v^{\prime}$, i.e. $\tilde{k}\left(x, v, v^{\prime}\right)=$ $\tilde{k}\left(x, v^{\prime}, v\right)$ for all $\left(x, v, v^{\prime}\right) \in \mathbb{T}^{d} \times \mathbb{R}^{d} \times \mathbb{R}^{d}$. Notice that for these kernels, $\mathbf{A 2}$ is automatically satisfied.

A classical example of such a kernel is the following.

E1'. Linear relaxation kernel. Taking $k\left(x, v, v^{\prime}\right)=\sigma(x) \mathcal{M}\left(v^{\prime}\right)$, with $\sigma \geq 0$, not identically vanishing and $\sigma \in C^{0}\left(\mathbb{T}^{d}\right)$ provides the simplest example of kernel in the class E1. This corresponds to the following equation (often called linearized BGK):

$$
\partial_{t} f+v \cdot \nabla_{x} f-\nabla_{x} V \cdot \nabla_{v} f=\sigma(x)\left(\left(\int_{\mathbb{R}^{d}} f d v\right) \mathcal{M}(v)-f\right) .
$$


This example also belongs to the following class.

E2. "Factorized" collision kernels Let $k$ be a collision kernel verifying A1-A3. We require $k$ to be of the form

$$
k\left(x, v, v^{\prime}\right)=\sigma(x) k^{*}\left(x, v, v^{\prime}\right)
$$

with $\sigma \in C^{0}\left(\mathbb{T}^{d}\right), \sigma \geq 0$, not identically vanishing and $k^{*} \in C^{0}\left(\mathbb{T}^{d} \times \mathbb{R}^{d} \times \mathbb{R}^{d}\right)$, satisfying for some $\lambda>0$, for all $x \in \mathbb{T}^{d}, v, v^{\prime} \in \mathbb{R}^{d}$,

$$
\frac{k^{*}\left(x, v^{\prime}, v\right)}{\mathcal{M}(v)}+\frac{k^{*}\left(x, v, v^{\prime}\right)}{\mathcal{M}\left(v^{\prime}\right)} \geq \lambda .
$$

The sub-class of $\mathbf{E 2}$ which is the most studied in the literature (see e.g. [20]) consists in the following non-degenerate case.

E2'. Non-degenerate collision kernels. Let $k$ be a collision kernel verifying A1-A3. The classical non-degeneracy condition consists in assuming that there exists $\lambda>0$ such that for all $x \in \mathbb{T}^{d}, v, v^{\prime} \in \mathbb{R}^{d}$

$$
\frac{k\left(x, v^{\prime}, v\right)}{\mathcal{M}(v)}+\frac{k\left(x, v, v^{\prime}\right)}{\mathcal{M}\left(v^{\prime}\right)}=\tilde{k}\left(x, v^{\prime}, v\right)+\tilde{k}\left(x, v, v^{\prime}\right) \geq \lambda
$$

Later in the paper (see Section 6), we will introduce other classes of collision kernels, that are interesting for our purposes.

Under assumptions A1-A3, the linear Boltzmann equation (1.1) is well-posed in appropriate Lebesgue spaces and some weighted $L^{2}$ norm of its solutions, that is the quantity

$$
\int_{\mathbb{T}^{d} \times \mathbb{R}^{d}}|f(t, x, v)|^{2} \frac{e^{V(x)}}{\mathcal{M}(v)} d v d x
$$

is dissipated (i.e. decreasing with respect to time, see Lemma 4.1).

This work aims at describing the large time behavior of solutions of (1.1), under assumptions A1-A3. This large framework aims at encompassing collision kernels $k$ which are degenerate in the following two senses:

- the collision kernel $k$ may vanish in a large subset of the phase space $\mathbb{T}^{d} \times \mathbb{R}^{d}$;

- we do not assume that $\tilde{k}$ is bounded below by a fixed positive constant at infinity in velocity.

However, still in the spirit of Villani's hypocercivity, one may hope that the transport term in (1.1) compensates for this strong degeneracy. Our goal is to find geometric criteria (on the hamiltonian $H$ and the collision kernel $k$ ) to:

- P1 characterize convergence to a global equilibrium,

- P1' characterize exponential convergence to this global equilibrium.

The study of these questions naturally leads to another problem: 
- P2 describe the structure and the localization properties of the spectrum of the underlying linear Boltzmann operator.

In recent works [4-6,16], Bernard, Desvillettes and Salvarani investigated P1 and P1' in a framework close to that of E2. In particular, in [5], the authors have shown that in the case where $V=0,(x, v) \in \mathbb{T}^{d} \times \mathbb{S}^{d-1}$, and $k^{*}\left(x, v, v^{\prime}\right)=k^{*}\left(v, v^{\prime}\right)$ (where $k^{*}$ is defined in E2), the exponential convergence to equilibrium (in the Lebesgue space $L^{1}$ ) was equivalent to a geometric control condition (similar to that of Bardos-LebeauRauch-Taylor in control theory [3,37]).

Previous works on this topic, for the non-degenerate class of collision kernels E2' include [33,38-41] (spectral approach), [10,17,20,35,42] (hypocoercivity methods), [28] (Lie techniques), and references therein. There are also several related works which concern the non-linear Boltzmann equation, but we do not mention them since that equation is not studied in this paper.

In this article, we introduce another point of view on these questions (in particular different from $[4-6,16])$, by implementing in this context different methods coming from control theory. We borrow several ideas from the seminal paper of Lebeau [32], which concerns the decay rates for the damped wave equation.

The goal of this paper is to give necessary and sufficient geometric conditions ensuring P1 and P1'. We also show that the methods we develop here are sufficiently robust to handle a general Riemannian setting.

In the companion paper [27], we shall explain how to adapt our methods for the case of specular reflection in bounded domains. The related question $\mathbf{P 2}$ will be studied as well in [26]. These results were announced in [25].

We now give a detailed overview of the main results of the present work.

\section{Overview of the Paper}

In this Section, we give an overview of the results contained in this paper. We state our results on the torus, i.e. when the phase space is $\mathbb{T}^{d} \times \mathbb{R}^{d}$, but they can all be generalized to Riemannian manifolds (see Sections 9 and 10).

\subsection{Some Definitions}

In this section, we introduce the notions needed to characterize convergence and exponential convergence to equilibrium. Given a collision kernel $k$ satisfying Assumptions A1-A3, we first introduce the set $\omega$ where the collisions are effective.

Definition 2.1 Define the open set of $\mathbb{T}^{d} \times \mathbb{R}^{d}$

$$
\omega:=\left\{(x, v) \in \mathbb{T}^{d} \times \mathbb{R}^{d}, \int_{\mathbb{R}^{d}} k\left(x, v, v^{\prime}\right) d v^{\prime}>0\right\}
$$


Note that because of A1-A2, we also have

$$
\begin{aligned}
\omega & =\left\{(x, v) \in \mathbb{T}^{d} \times \mathbb{R}^{d}, \exists v^{\prime} \in \mathbb{R}^{d}, k\left(x, v, v^{\prime}\right)>0\right\} \\
& =\left\{(x, v) \in \mathbb{T}^{d} \times \mathbb{R}^{d}, \exists v^{\prime} \in \mathbb{R}^{d}, k\left(x, v^{\prime}, v\right)>0\right\} .
\end{aligned}
$$

Let us recall the definition of the hamiltonian flow associated to $H$, and associated characteristic curves (or characteristics) in the present setting.

Definition 2.2 The hamiltonian flow $\left(\phi_{t}\right)_{t \in \mathbb{R}}$ associated to $H(x, v)=\frac{|v|^{2}}{2}+V(x)$ is the one parameter group of diffeomorphisms on $\mathbb{T}^{d} \times \mathbb{R}^{d}$ defined by $\phi_{t}(x, v):=$ $\left(X_{t}(x, v), \Xi_{t}(x, v)\right)$ with $(x, v) \in \mathbb{T}^{d} \times \mathbb{R}^{d}$ and

$$
\left\{\begin{array}{l}
\frac{d X_{t}(x, v)}{d t}=\Xi_{t}(x, v), \\
\frac{d \Xi_{t}(x, v)}{d t}=-\nabla_{x} V\left(X_{t}(x, v)\right), \\
X_{t=0}=x, \quad \Xi_{t=0}=v .
\end{array}\right.
$$

The characteristic curve stemming from $(x, v) \in \mathbb{T}^{d} \times \mathbb{R}^{d}$ is the curve $\left\{\phi_{t}(x, v), t \in\right.$ $\left.\mathbb{R}^{+}\right\}$.

Recall that throughout the paper, we assume that $V \in W^{2, \infty}\left(\mathbb{T}^{d}\right)$, so that the Cauchy-Lipshitz theorem ensures the local existence and uniqueness of the solutions of (2.3). Global existence follows from the fact that $H$ is preserved along any characteristic curve. Note in particular that each energy level $\{H=R\}$ is compact ( $V$ being continuous on $\mathbb{T}^{d}$, it is bounded from below). Hence, each characteristic curve is contained in a compact set of $\mathbb{T}^{d} \times \mathbb{R}^{d}$.

The notions needed to understand the interaction between collisions and transport are of two different nature. We start by expressing purely geometric definitions. Then, we formulate structural-geometric definitions. We finally introduce the weighted Lebesgue spaces used in this paper, as well as a definition of a unique continuation type property.

\subsubsection{Geometric Definitions}

We start by introducing the following definitions:

- The Geometric Control Condition of [3,37], in Definition 2.3,

- The Lebeau constants of [32], $C^{-}(\infty)$ and $C^{+}(\infty)$, in Definition 2.4,

- The almost everywhere in infinite time Geometric Control Condition, in Definition 2.5.

Let us first recall the Geometric Control Condition, which is a classical notion in the context of control theory. It is due to Rauch-Taylor [37] and Bardos-LebeauRauch [3]. 
Definition 2.3 Let $U$ be an open subset of $\mathbb{T}^{d} \times \mathbb{R}^{d}$ and $T>0$. We say that $(U, T)$ satisfies the Geometric Control Condition (GCC) with respect to the hamiltonian $H(x, v)=\frac{|v|^{2}}{2}+V(x)$ if for any $(x, v) \in \mathbb{T}^{d} \times \mathbb{R}^{d}$, there exists $t \in[0, T]$ such that $\phi_{t}(x, v)=\left(X_{t}(x, v), \Xi_{t}(x, v)\right) \in U$.

We shall say that $U$ satisfies the Geometric Control Condition with respect to the hamiltonian $H(x, v)=\frac{|v|^{2}}{2}+V(x)$ if there exists $T>0$ such that the couple $(U, T)$ does.

We now define two important constants in view of the study of the large time behavior of the linear Boltzmann equation, which involve averages of the damping function (usually called collision frequency in kinetic theory) $b(x, v):=\int_{\mathbb{R}^{d}} k\left(x, v, v^{\prime}\right) d v^{\prime}$ along the flow $\phi_{t}$.

Definition 2.4 Define the Lebeau constants ([32]) in $\mathbb{R}^{+} \cup\{+\infty\}$ by

$$
\begin{aligned}
& C^{-}(\infty):=\sup _{T \in \mathbb{R}^{+}} C^{-}(T), \\
& C^{-}(T)=\inf _{(x, v) \in \mathbb{T}^{d} \times \mathbb{R}^{d}} \frac{1}{T} \int_{0}^{T}\left(\int_{\mathbb{R}^{d}} k\left(\phi_{t}(x, v), v^{\prime}\right) d v^{\prime}\right) d t, \\
& C^{+}(\infty):=\inf _{T \in \mathbb{R}^{+}} C^{+}(T), \\
& C^{+}(T)=\sup _{(x, v) \in \mathbb{T}^{d} \times \mathbb{R}^{d}} \frac{1}{T} \int_{0}^{T}\left(\int_{\mathbb{R}^{d}} k\left(\phi_{t}(x, v), v^{\prime}\right) d v^{\prime}\right) d t,
\end{aligned}
$$

where $\phi_{t}$ denotes the hamiltonian flow of Definition 2.2.

It is not clear at first sight that $C^{-}(\infty)$ and $C^{+}(\infty)$ are well defined: see [32] and the beginning of Section 7 for a short explanation. It turns out that only $C^{-}(\infty)$ will be useful in this paper (but $C^{+}(\infty)$ will be interesting in the companion paper [26]).

Finally, we introduce a weaker version of the Geometric Control Condition, which will also plays an important role in this work.

Definition 2.5 Let $U$ be an open subset of $\mathbb{T}^{d} \times \mathbb{R}^{d}$. We say that $U$ satisfies the almost everywhere infinite time (a.e.i.t.) Geometric Control Condition with respect to the hamiltonian $H(x, v)=\frac{|v|^{2}}{2}+V(x)$ if for almost every $(x, v) \in \mathbb{T}^{d} \times \mathbb{R}^{d}$, there exists $s \geq 0$ such that the characteristics $\left(X_{t}(x, v), \Xi_{t}(x, v)\right)_{t \geq 0}$ associated to $H$ satisfy $\left(X_{t=s}, \Xi_{t=s}\right) \in U$.

Using this terminology, the usual Geometric Control Condition of Definition 2.3 could be called "everywhere finite time" GCC.

Remark 2.1 We have the following characterization of the different geometric properties introduced here.

- The couple $(U, T)$ satisfies GCC if and only if $\bigcup_{s \in(0, T)} \phi_{-s}(U)=\mathbb{T}^{d} \times \mathbb{R}^{d}$.

- The set $U$ satisfies the a.e.i.t. GCC if and only if there exists $\mathcal{N} \subset \mathbb{T}^{d} \times \mathbb{R}^{d}$ with zero Lebesgue measure such that $\bigcup_{s \in \mathbb{R}^{+}} \phi_{-s}(U) \cup \mathcal{N}=\mathbb{T}^{d} \times \mathbb{R}^{d}$. Note that this implies in particular that $\mathcal{N}$ is a closed subset of $\mathbb{T}^{d} \times \mathbb{R}^{d}$ satisfying $\phi_{s}(\mathcal{N}) \subset \mathcal{N}$ for all $s \geq 0$. 


\subsubsection{Structural-Geometric Definitions}

In the sequel, the above geometric definitions are used with $U=\omega$. They hence involve joint properties of the flow $\phi_{t}$ together with the open set $\omega$, i.e. of the damping function $b=\int_{\mathbb{R}^{d}} k\left(\cdot, \cdot, v^{\prime}\right) d v^{\prime}$. As such, they do not take into account the fine structure of the Boltzmann operator, and in particular the non-local property with respect to the velocity variable of the gain operator $f \mapsto\left((x, v) \mapsto \int_{\mathbb{R}^{d}} k\left(x, v^{\prime}, v\right) f\left(x, v^{\prime}\right) d v^{\prime}\right)$.

The next definitions aim at describing how the information may travel between the different connected components of $\omega$. Let us first define two basic binary relations on the open sets of $\mathbb{T}^{d} \times \mathbb{R}^{d}$.

Definition 2.6 Let $U_{1}$ and $U_{2}$ be two open subsets of $\mathbb{T}^{d} \times \mathbb{R}^{d}$. We say that $U_{1} \mathcal{R}_{\phi} U_{2}$ if there exist $s \in \mathbb{R}$ such that $\phi_{s}\left(U_{1}\right) \cap U_{2} \neq \emptyset$.

Definition 2.7 Let $U_{1}$ and $U_{2}$ be two open subsets of $\mathbb{T}^{d} \times \mathbb{R}^{d}$. We say that $U_{1} \mathcal{R}_{k} U_{2}$ if there exist $\left(x, v_{1}, v_{2}\right) \in \mathbb{T}^{d} \times \mathbb{R}^{d} \times \mathbb{R}^{d}$ with $\left(x, v_{1}\right) \in U_{1},\left(x, v_{2}\right) \in U_{2}$ such that $k\left(x, v_{1}, v_{2}\right)>0$ or $k\left(x, v_{2}, v_{1}\right)>0$.

Both relations are symmetric and $\mathcal{R}_{\phi}$ is moreover reflexive. When restricted to open sets intersecting $\omega$, the relation $\mathcal{R}_{k}$ also becomes reflexive. The relation $\mathcal{R}_{\phi}$ expresses the fact that the open sets are "connected through" the flow $\phi_{s}$, whereas the relation $\mathcal{R}_{k}$ means that the open sets are "connected through" a collision.

We also define another convenient $x$-dependent binary relation.

Definition 2.8 Let $x \in \mathbb{T}^{d}$ and $O_{1}$ and $O_{2}$ be two open subsets of $\mathbb{R}^{d}$. We say that $O_{1} \mathcal{R}_{k}^{x} O_{2}$ if there exists $\left(v_{1}, v_{2}\right) \in \mathbb{R}^{d} \times \mathbb{R}^{d}$ with $v_{1} \in O_{1}, v_{2} \in O_{2}$ such that $k\left(x, v_{1}, v_{2}\right)>0$ or $k\left(x, v_{2}, v_{1}\right)>0$.

Given now an open subset $U$ of $\mathbb{T}^{d} \times \mathbb{R}^{d}$, we define $U(x)=\left\{v \in \mathbb{R}^{d},(x, v) \in U\right\}$. With this notation, notice that $U_{1} \mathcal{R}_{k} U_{2}$ if and only if there exists $x \in \mathbb{T}^{d}$ such that $U_{1}(x) \mathcal{R}_{k}^{x} U_{2}(x)$.

Given $U$ an open set of $\mathbb{T}^{d} \times \mathbb{R}^{d}$, we denote by $\mathcal{C C}(U)$ the set of connected components of $U$. Note that from the separability of $\mathbb{T}^{d} \times \mathbb{R}^{d}$, it follows that for any open set $U \subset \mathbb{T}^{d} \times \mathbb{R}^{d}$, the cardinality of the set $\mathcal{C C}(U)$ is at most countable.

In the sequel, the main open sets $U$ we are interested in are $\omega$ and $\bigcup_{s \in \mathbb{R}^{+}} \phi_{-s}(\omega)$. We now define the key equivalence relation on $\mathcal{C C}(\omega)$.

Definition 2.9 Given $\omega_{1}$ and $\omega_{2}$ two connected components of $\omega$, we say that $\omega_{1} \approx \omega_{2}$ if there are $N \in \mathbb{N}^{*}$ and $N$ connected components $\left(\omega^{(i)}\right)_{1 \leq i \leq N}$ of $\omega$ such that

- we have $\omega_{1} \mathcal{R}_{\phi} \omega^{(1)}$ or $\omega_{1} \mathcal{R}_{k} \omega^{(1)}$,

- for all $1 \leq i \leq N-1$, we have $\omega^{(i)} \mathcal{R}_{\phi} \omega^{(i+1)}$ or $\omega^{(i)} \mathcal{R}_{k} \omega^{(i+1)}$,

- we have $\omega^{(N)} \mathcal{R}_{\phi} \omega_{2}$ or $\omega^{(N)} \mathcal{R}_{k} \omega_{2}$.

The relation $\approx$ is an equivalence relation on the $\operatorname{set} \mathcal{C C}(\omega)$ of connected components of $\omega$. For $\omega_{1} \in \mathcal{C C}(\omega)$, we denote its equivalence class for $\approx$ by [ $\left.\omega_{1}\right]$.

This definition means that the two connected components $\omega_{1}$ and $\omega_{2}$ are linked by $\mathcal{R}_{\phi}$ or $\mathcal{R}_{k}$ through a chain of connected components of $\omega$.

Remark 2.2 We will introduce later in Section 3.2 a related equivalence relation on $\mathcal{C C}\left(\bigcup_{t \geq 0} \phi_{-t} \omega\right)$. 


\subsubsection{Weighted Lebesgue Spaces and a Unique Continuation Type Property}

Let us now introduce the weighted Lebesgue spaces that will be used throughout this paper.

Definition 2.10 (Weighted $L^{p}$ spaces). We define the Banach spaces $\mathcal{L}^{p}\left(\mathbb{T}^{d} \times \mathbb{R}^{d}\right)$ (for $p \in[1,+\infty))$ and $\mathcal{L}^{\infty}\left(\mathbb{T}^{d} \times \mathbb{R}^{d}\right)$ by

$$
\begin{aligned}
& \mathcal{L}^{p}\left(\mathbb{T}^{d} \times \mathbb{R}^{d}\right):=\left\{f \in L_{l o c}^{1}\left(\mathbb{T}^{d} \times \mathbb{R}^{d}\right), \int_{\mathbb{T}^{d} \times \mathbb{R}^{d}}|f|^{p} \frac{e^{V}}{\mathcal{M}(v)} d v d x<+\infty\right\}, \\
& \|f\|_{\mathcal{L}^{p}}=\left(\int_{\mathbb{T}^{d} \times \mathbb{R}^{d}}|f|^{p} \frac{e^{V}}{\mathcal{M}(v)} d v d x\right)^{1 / p} . \\
& \mathcal{L}^{\infty}\left(\mathbb{T}^{d} \times \mathbb{R}^{d}\right):=\left\{f \in L_{l o c}^{1}\left(\mathbb{T}^{d} \times \mathbb{R}^{d}\right), \sup _{\mathbb{T}^{d} \times \mathbb{R}^{d}}|f| \frac{e^{V}}{\mathcal{M}(v)}<+\infty\right\}, \\
& \|f\|_{\mathcal{L}^{\infty}}=\sup _{\mathbb{T}^{d} \times \mathbb{R}^{d}}|f| \frac{e^{V}}{\mathcal{M}(v)} .
\end{aligned}
$$

The space $\mathcal{L}^{2}$ is a (real) Hilbert space endowed with the inner product

$$
\langle f, g\rangle_{\mathcal{L}^{2}}:=\int_{\mathbb{T}^{d} \times \mathbb{R}^{d}} e^{V} \frac{f g}{\mathcal{M}(v)} d v d x
$$

We finally define a Unique Continuation Property for (1.1).

Definition 2.11 We say that the set $\omega$ satisfies the Unique Continuation Property if the only solution $f \in C_{t}^{0}\left(\mathcal{L}^{2}\right)$ to

$$
\left\{\begin{array}{l}
\partial_{t} f+v \cdot \nabla_{x} f-\nabla_{x} V \cdot \nabla_{v} f=0 \\
\mathscr{C}(f)=0
\end{array}\right.
$$

is $f=\left(\int_{\mathbb{T}^{d} \times \mathbb{R}^{d}} f d v d x\right) e^{-V} \mathcal{M}$.

It is actually possible to reformulate in a more explicit form the second equation in (2.6), involving the value of $f$ on connected components of $\omega$ (see Remark 4.1).

\subsection{Convergence to Equilibrium}

Recall that the main goal of this paper is to provide necessary and sufficient geometric conditions to ensure P1 and P1'. Our results can be formulated as follows.

We first give a general characterization of convergence to some equilibrium.

Theorem 2.1 The following statements are equivalent.

(1) The set $\omega$ satisfies the a.e.i.t. GCC with respect to $H$. 
(2) For all $f_{0} \in \mathcal{L}^{2}$, there exists a stationary solution $P f_{0}$ of (1.1) such that

$$
\left\|f(t)-P f_{0}\right\|_{\mathcal{L}^{2}} \rightarrow_{t \rightarrow+\infty} 0,
$$

where $f(t)$ is the solution of (1.1) with initial datum $f_{0}$.

Theorem 2.1 is actually a weak version of Theorem 5.1, which is our main result in this direction. If (1) or (2) holds, we can actually describe precisely the stationary solution $P f_{0}$. Note that this description is a key step of the proof of Theorem 5.1 (and thus, that of Theorem 2.1). It involves the equivalence classes of another equivalence relation, which is related to $\approx$ (see Definition 3.1 and Lemma 3.1): we refer to the statement of Theorem 5.1. In particular, in several cases, the stationary solution $P f_{0}$ is not the Maxwellian equilibrium

$$
\left(\int_{\mathbb{T}^{d} \times \mathbb{R}^{d}} f_{0} d v d x\right) e^{-V(x)} \mathcal{M}(v) .
$$

As a matter of fact, we will see that the dimension of the vector space of stationary solutions is equal to the number of equivalence classes for $\approx$. An explicit example of linear Boltzmann equation with several equivalence classes for $\approx$ (and thus, for which $P f_{0}$ is not given by (2.7)) is exhibited in Section 6.2.

Among all possible stationary solutions of the linear Boltzmann equation, the Maxwellian equilibrium (2.7) of course particularly stands out. In the next theorem (which is actually also a particular case of Theorem 5.1), we characterize the situation for which the stationary solution ultimately reached is precisely the projection to the Maxwellian.

\section{Theorem 2.2 The following statements are equivalent.}

(i.) The set $\omega$ satisfies the Unique Continuation Property.

(ii.) The set $\omega$ satisfies the a.e.i.t. GCC and there exists one and only one equivalence class for $\approx$.

(iii.) For all $f_{0} \in \mathcal{L}^{2}\left(\mathbb{T}^{d} \times \mathbb{R}^{d}\right)$, denote by $f(t)$ the unique solution to (1.1) with initial datum $f_{0}$. We have

$$
\left\|f(t)-\left(\int_{\mathbb{T}^{d} \times \mathbb{R}^{d}} f_{0} d v d x\right) e^{-V(x)} \mathcal{M}\right\|_{\mathcal{L}^{2}} \rightarrow_{t \rightarrow+\infty} 0 .
$$

As already mentioned in the introduction, our proofs are inspired by ideas which originate from control theory. For the sake of brevity, we shall not give a detailed explanation of the proof of these results in this introduction. Nevertheless, we would like to comment on an important aspect of the proof of (i.) implies (iii.) in Theorem 2.2. Our approach is based on the fact that the square of the $\mathcal{L}^{2}$ norm of a solution $f(t)$ of (1.1), which we shall sometimes refer to as the energy, is damped via an explicit dissipation identity, see Lemma 4.1:

$$
\text { for all } t \geq 0, \quad \frac{d}{d t}\|f(t)\|_{\mathcal{L}^{2}}^{2}=-\mathscr{D}(f)
$$


with $\mathscr{D}(f) \geq 0$, which we shall call the dissipation term.

The idea of the proof is to assume by contradiction that there exists an initial condition $g_{0}$ in $\mathcal{L}^{2}$, with zero mean, such that the associated solution $g(t)$ to $(1.1)$ does not decay to 0 . This yields the existence of $\varepsilon>0$ and of a sequence of times $\left(t_{n}\right)_{n \geq 0}$ going to $+\infty$ such that $\left\|g\left(t_{n}\right)\right\|_{\mathcal{L}^{2}} \geq \varepsilon$.

We then study the sequence of shifted functions $h_{n}(t):=g\left(t+t_{n}\right)$. This is the core of our analysis, which basically consists in a uniqueness-compactness argument. We study the weak limit of $h_{n}$ and show, using the identity (2.9) and the unique continuation property, that it is necessarily trivial. Then, we consider the associated sequence of defect measures and prove that it is also necessarily trivial, yielding a contradiction.

A difficulty in the analysis comes from the fact that in general, the dissipation term does not control neither the $\mathcal{L}^{2}$ distance to the projection on the set of stationary solutions, nor the $\mathcal{L}^{2}$ norm of the collision operator. However, what holds true is the weak coercivity property

$$
\text { for } f \in \mathcal{L}^{2}, \quad \mathscr{D}(f)=0 \Longrightarrow \mathscr{C}(f)=0 \text {, }
$$

see Lemma 4.5. This turns out to be sufficient for our needs. Denoting by

$$
A:=T-\mathscr{C}
$$

the linear Boltzmann operator, where $T f=\left(v \cdot \nabla_{x}-\nabla_{x} V \cdot \nabla_{v}\right) f$ and $\mathscr{C}$ is the collision operator, the property (2.10) together with the skew-adjointness of $T$ then implies that $\operatorname{Ker}(A)=\operatorname{Ker}(T) \cap \operatorname{Ker}(\mathscr{C})$. This precise structure, together with the equivalence relation $\approx$, allows to identify $\operatorname{Ker}(A)$, i.e. the space of stationary solutions of (1.1).

Besides, when studying defect measures, the analysis relies on another peculiar structure of (1.1), which is, loosely speaking, made of a propagative and dissipative part (transport and the loss term) and a relatively compact part (the gain term). That the gain term is relatively compact is proved via averaging lemmas (see Appendix 2 and the references therein).

\subsection{Exponential Convergence to Equilibrium}

For what concerns exponential convergence, we need to introduce a technical assumption, which implies an additional mild growth condition on the kernel $k$ at infinity, with respect to A3:

A3'. Assume that there exists a continuous function $\varphi(x, v):=\Theta \circ H(x, v)$ with $\Theta: \mathbb{R} \rightarrow[1,+\infty)$, such that for all $(x, v) \in \mathbb{T}^{d} \times \mathbb{R}^{d}$, we have

$$
\int_{\mathbb{R}^{d}} k\left(x, v, v^{\prime}\right) d v^{\prime} \leq \varphi(x, v),
$$


and

$$
\sup _{x \in \mathbb{T}^{d}} \int_{\mathbb{R}^{d} \times \mathbb{R}^{d}} k^{2}\left(x, v^{\prime}, v\right) \frac{\mathcal{M}\left(v^{\prime}\right)}{\mathcal{M}(v)}\left(\frac{\varphi(x, v)}{\varphi\left(x, v^{\prime}\right)}-1\right)^{2} d v d v^{\prime}<+\infty
$$

As for A3, this assumption is satisfied in the standard case where $\tilde{k}$ has a polynomial growth in the variables $v$ and $v^{\prime}$. Once $\mathbf{A} \mathbf{3}$ is assumed, it can be rephrased as

$$
\begin{aligned}
& \int_{\mathbb{R}^{d}} k\left(x, v, v^{\prime}\right) d v^{\prime} \leq \varphi(x, v), \\
& \sup _{x \in \mathbb{T}^{d}} \int_{\mathbb{R}^{d} \times \mathbb{R}^{d}} k^{2}\left(x, v^{\prime}, v\right) \frac{\mathcal{M}\left(v^{\prime}\right)}{\mathcal{M}(v)} \frac{\varphi(x, v)^{2}}{\varphi\left(x, v^{\prime}\right)^{2}} d v d v^{\prime}<+\infty .
\end{aligned}
$$

The assumption is satisfied by kernels $k\left(x, v, v^{\prime}\right)=\tilde{k}\left(x, v, v^{\prime}\right) \mathcal{M}\left(v^{\prime}\right)$ for instance as soon as we have a bound of the form

$$
\tilde{k}\left(x, v, v^{\prime}\right) \leq \lambda e^{\varepsilon_{1}|v|^{2}+\varepsilon_{2}\left|v^{\prime}\right|^{2}}, \quad \text { with } \varepsilon_{1}+\varepsilon_{2}<\frac{1}{4}, \quad \lambda>0,
$$

with $\varphi=\Theta \circ H$ and $\Theta(t)=C e^{2 \max \left\{\varepsilon_{1}, 0\right\} t}, C$ large enough. Furthermore, for some $C_{0}, C_{1}>0$, we have $C_{0} e^{\max \left\{\varepsilon_{1}, 0\right\}|v|^{2}} \leq \varphi(x, v) \leq C_{1} e^{\max \left\{\varepsilon_{1}, 0\right\}|v|^{2}}$. Of course, in the case $\varepsilon_{1}+\varepsilon_{2}=\frac{1}{4}$, this can be refined with a polynomial correction.

We have the following criterion, assuming that A3' is satisfied in addition to A1-A3.

Theorem 2.3 (Exponential convergence to equilibrium). Assume that the collision kernel satisfies A3'. The following statements are equivalent:

(a.) $C^{-}(\infty)>0$.

(b.) There exist $C>0, \gamma>0$ such that for any $f_{0} \in \mathcal{L}^{2}\left(\mathbb{T}^{d} \times \mathbb{R}^{d}\right)$, the unique solution to (1.1) with initial datum $f_{0}$ satisfies for all $t \geq 0$,

$$
\begin{aligned}
& \left\|f(t)-\left(\int_{\mathbb{T}^{d} \times \mathbb{R}^{d}} f_{0} d v d x\right) e^{-V(x)} \mathcal{M}\right\|_{\mathcal{L}^{2}} \\
& \leq C e^{-\gamma t}\left\|f_{0}-\left(\int_{\mathbb{T}^{d} \times \mathbb{R}^{d}} f_{0} d v d x\right) e^{-V(x)} \mathcal{M}\right\|_{\mathcal{L}^{2}} .
\end{aligned}
$$

(c.) There exists $C>0, \gamma>0$ such that for any $f_{0} \in \mathcal{L}^{2}\left(\mathbb{T}^{d} \times \mathbb{R}^{d}\right)$, there exists a stationary solution $P f_{0}$ of (1.1) such that the unique solution to (1.1) with initial datum $f_{0}$ satisfies for all $t \geq 0$,

$$
\left\|f(t)-P f_{0}\right\|_{\mathcal{L}^{2}} \leq C e^{-\gamma t}\left\|f_{0}-P f_{0}\right\|_{\mathcal{L}^{2}}
$$

Remark 2.3 If we do not assume that $\mathbf{A 3}$ ' is satisfied, then we still have that (a.) implies (b.) and (c.). 
If $C^{-}(\infty)>0$, note in particular that the Geometric Control Condition of Definition 2.3 is satisfied.

One interesting consequence of Theorem 2.3 is a rigidity property of the Maxwellian equilibrium with respect to exponential convergence: loosely speaking, given a linear Boltzmann equation with a collision kernel satisfying A1-A2-A3-A3', if uniform convergence to (some) equilibrium holds, then the stationary solution ultimately reached is necessarily the projection of the initial datum on the Maxwellian equilibrium.

The assumption A3' is used in the proof of $(b.) \Longrightarrow(a$.) to solve an issue due to the combined effect of the nonlocality of the operator $\mathscr{C}^{+}$(which, from small velocities, can instantaneously create arbitrary large ones), together with the unboundedness of the multiplication operator $\mathscr{C}^{-}$(still for large velocities).

As an immediate consequence of Theorem 2.3, we deduce the following result.

Corollary 2.1 Assume that there is $x \in \mathbb{T}^{d}$ such that $\nabla_{x} V(x)=0$ and $\int k\left(x, 0, v^{\prime}\right) d v^{\prime}=0$. Then $C^{-}(\infty)=0$ and there is no uniform exponential rate of convergence to equilibrium.

In particular we get in the free transport case:

Corollary 2.2 Assume that $V=0$ and that $p_{x}(\omega) \neq \mathbb{T}^{d}$, where

$$
p_{x}(\omega)=\left\{x \in \mathbb{T}^{d} \text {, there exists } v \in \mathbb{R}^{d} \text { such that }(x, v) \in \omega\right\}
$$

denotes the projection on the space of positions. Then there is no uniform exponential rate of convergence to equilibrium.

The proof of Theorem 2.3 is as well inspired by ideas coming from control theory. The proof of $(a.) \Longrightarrow(b$.) relies on the following facts.

- By (2.9), the exponential decay (i.e. (ii.) in Theorem 2.3) can be rephrased as a certain observability inequality relating the dissipation and the energy at time 0 , see Lemma 11.1: there exist $K, T>0$ such that for all $f_{0} \in \mathcal{L}^{2}\left(\mathbb{T}^{d} \times \mathbb{R}^{d}\right)$ with zero mean, we have

$$
K \int_{0}^{T} \mathscr{D}(f) d t \geq\left\|f_{0}\right\|_{\mathcal{L}^{2}}^{2},
$$

where $f$ is the solution of (1.1) with initial datum $f_{0}$.

- This inequality is proved using a contradiction argument, following Lebeau [32], which also consists in a uniqueness-compactness argument. The analysis follows the same lines as those of (i.) implies (iii.) in the proof of Theorem 2.2. In particular, it also relies on the weak coercivity property (2.10). The main difference is that we need to use here the fact that the Lebeau constant is positive in order to show that the sequence of defect measures becomes trivial at the limit, yielding a contradiction.

For what concerns $(b.) \Longrightarrow(a$.), the idea is to contradict the observability condition: we construct a sequence of initial conditions in $\mathcal{L}^{2}\left(\mathbb{T}^{d} \times \mathbb{R}^{d}\right)$ for (1.1), which concentrate to a trapped ray (whose existence is guaranteed by the cancellation 
of the Lebeau constant). Loosely speaking, this corresponds to a geometric optics type construction. In order to justify this procedure, we need that the collision kernel satisfies A3'. Finally, we mention that the proof of $(c.) \Longrightarrow(a$.) is similar but relies on an additional argument based on the precise version of Theorem 2.1.

Remarks 2.1 (1) As for the damped wave equation [3,31,32,37], the study of asymptotic decay rates relies on "phase space" analysis. However, as opposed to the wave equation, the Boltzmann equation is directly set on the phase space. As a consequence, the study of associated propagation and damping phenomena only uses "local" analysis, whereas that of the wave equation (see $[3,31,32,37]$ ) requires the use of microlocal analysis.

(2) One technical difficulty here is to handle the lack of compactness of the phase space $\mathbb{T}^{d} \times \mathbb{R}^{d}$ in the variable $v$. It is also possible to consider the equations on a compact phase space. In this case, all our proofs apply, sometimes with significant simplifications. We refer to Section 10.

\subsection{Organization of the Paper}

The main part of this paper is dedicated to the proof of Theorems 2.1, 2.2 and 2.3. Before this, we start with two preliminary sections, namely Sections 3 and 4. In Section 3, we provide several comments on the results, the assumptions, and illustrate some geometric definitions; we also introduce another key equivalence relation used in the main proofs. In Section 4, we then give some preliminaries in the analysis; in Section 4.1, we start by proving the well-posedness of (1.1) and the associated dissipation identity, while Section 4.2 is dedicated to a detailed study of the kernel of the collision operator, which leads to the weak coercivity property (2.10). Section 5 is mainly devoted to the proofs of Theorems 2.2 and 2.1; in Section 5.1, we start by proving Theorem 2.2. Then, in Section 5.2, we state and prove Theorem 5.1, which is the precise version of Theorem 2.1. Section 6 is dedicated to the application of these results to some particular classes of collision kernels. In Section 7, we prove Theorem 2.3. In Section 8, we briefly revisit the recent work of Bernard and Salvarani [4] in our framework, in order to give some abstract lower bounds on the convergence rate when $C^{-}(\infty)=0$. Finally, we adapt our analysis in order to handle other geometric situations. In Section 9, we deal with the case of a general compact Riemannian manifold (without boundary): we first explain how to express the linear Boltzmann equation in this setting and generalize Theorems 5.1 and 2.3 to this context. Also, in Section 10, we explain very shortly how to adapt all these results to the case of compact phase spaces.

This paper ends with five appendices. In Appendix 1, we give the equivalence between exponential decay and the observability inequality, used crucially in the proof of Theorem 2.3. In Appendix 2 we give a reminder about classical averaging Lemmas and adapt them to our purposes. In Appendix 3, we provide reformulations of some geometric properties. In Appendix 4, we provide the proof of Proposition 3.2, which relates the two equivalence relations, which are key notions in our analysis. In Appendix 5, to stress the robustness of our methods, we explain how the results of this paper 
concerning large time behavior can be adapted to other Boltzmann-like equations (e.g. relativistic Boltzmann equation or general linearized BGK equation).

Note added in proof After completion of this paper, we learnt about the work of Mokhtar-Karroubi [34] who studied question P1' in the case where $V=0,(x, v) \in$ $\mathbb{T}^{d} \times U$, and $U$ is a bounded open set of $\mathbb{R}^{d}$; he also introduced some quantities in his analysis which are the same as the Lebeau constants.

\section{Remarks and Examples}

In this section, we provide several comments on the different geometric definitions introduced in Sections 2.1.1 and 2.1.2.

\subsection{About a.e.i.t. GCC in the Torus}

A first natural question is to understand the a.e.i.t. GCC in the usual situation of free transport (i.e. $V=0$ ), when $\omega$, i.e. the set where collisions are effective, is of the simple form $\omega_{x} \times \mathbb{R}^{d}$. We prove that this condition is satisfied for any nonempty $\omega_{x} \subset \mathbb{T}^{d}$. We also prove that this situation is very particular, and unstable with respect to small perturbations of the potential.

Proposition 3.1 Suppose that $V=0$ and that $\omega=\omega_{x} \times \mathbb{R}^{d}$, where $\omega_{x}$ is a non-empty open subset of $\mathbb{T}^{d}$. Then (i.) - (ii.) - (iii.) in Theorem 2.2 hold.

Such a result is in particular relevant for the study of the linearized BGK equation (class E1'). Proposition 3.1 shows that there is convergence to the Maxwellian equilibrium (2.7) as soon as $\sigma$ does not vanish identically.

On the other hand, $\omega$ being fixed, we give an example of dynamics (i.e. exhibit a potential $V$ ) for which the a.e.i.t. GCC fails. More precisely, we prove that this property is very unstable with respect to small perturbations of the potential: for $\omega=\omega_{x} \times \mathbb{R}^{d} \neq \mathbb{T}^{d} \times \mathbb{R}^{d}$ there exist arbitrary small potentials (in any $C^{k}$-norm) such that $\omega$ does not satisfy a.e.i.t. GCC for the associated Hamiltonian. This illustrates the fact that the dynamics associated to free transport in the torus is not generic.

Proposition 3.2 Assume that $\overline{p_{x}(\omega)} \neq \mathbb{T}^{d}$, where $p_{x}(\omega)$ denotes the projection of $\omega$ on $\mathbb{T}^{d}$ defined in (2.14). Then there exists a potential $V \in C^{\infty}\left(\mathbb{T}^{d}\right)$ such that for any $\varepsilon>0$, $\omega$ does not satisfy a.e.i.t. GCC for the Hamiltonian $H_{\varepsilon}(x, v)=\frac{|v|^{2}}{2}+\varepsilon V(x)$.

The proof of Proposition 3.1 and Proposition 3.2 are given respectively in Section 6.3 and Appendix 4.

\subsection{The Equivalence Relation on $\mathcal{C C}\left(\bigcup_{t \geq 0} \phi_{-t}(\omega)\right)$}

We define here another key equivalence relation $\sim$ on the set of connected components of $\bigcup_{s \in \mathbb{R}^{+}} \phi_{-s}(\omega)$. We then explain the link between the two equivalence relations $\sim$ on $\mathcal{C C}\left(\bigcup_{t \geq 0} \phi_{-t}(\omega)\right)$ and $\approx$ on $\mathcal{C C}(\omega)$. 
Definition 3.1 Given $\Omega_{1}, \Omega_{2}$ two connected components of $\bigcup_{s \in \mathbb{R}^{+}} \phi_{-s}(\omega)$, we say that $\Omega_{1} \sim \Omega_{2}$ if there are $N \in \mathbb{N}$ and $N$ connected components $\left(\Omega^{i}\right)_{1 \leq i \leq N}$ of $\bigcup_{s \in \mathbb{R}^{+}} \phi_{-s}(\omega)$ such that

- we have $\Omega_{1} \mathcal{R}_{k} \Omega^{(1)}$,

- for all $1 \leq i \leq N-1$, we have $\Omega^{(i)} \mathcal{R}_{k} \Omega^{(i+1)}$,

- we have $\Omega^{(N)} \mathcal{R}_{k} \Omega_{2}$.

The relation $\sim$ is an equivalence relation on the set of $\mathcal{C C}\left(\bigcup_{s \in \mathbb{R}^{+}} \phi_{-s}(\omega)\right)$ of connected components of $\bigcup_{s \in \mathbb{R}^{+}} \phi_{-s}(\omega)$. For $\Omega_{1} \in \mathcal{C C}\left(\bigcup_{s \in \mathbb{R}^{+}} \phi_{-s}(\omega)\right)$, we denote its equivalence class for $\sim$ by $\left[\Omega_{1}\right]$.

The following lemma gives the link between the two equivalence relations. We define the function

$$
\begin{aligned}
\Psi: \mathcal{C C}(\omega) & \rightarrow \mathcal{C C}\left(\bigcup_{s \in \mathbb{R}^{+}} \phi_{-s}(\omega)\right) \\
\omega_{0} & \mapsto \Omega_{0}, \text { such that } \omega_{0} \subset \Omega_{0} .
\end{aligned}
$$

The application $\Psi$ maps $\omega_{0} \in \mathcal{C C}(\omega)$ to the connected component $\Omega_{0}$ of $\bigcup_{s \in \mathbb{R}^{+}} \phi_{-s}(\omega)$ containing $\omega_{0}$.

Lemma 3.1 Given $\omega_{1}, \omega_{2} \in \mathcal{C C}(\omega)$, we have $\omega_{1} \approx \omega_{2}$ if and only if $\Psi\left(\omega_{1}\right) \sim$ $\Psi\left(\omega_{2}\right)$. As a consequence, $\Psi$ goes to the quotient defining a bijection $\tilde{\Psi}$ between the equivalence classes of $\approx$ and $\sim$ :

$$
\tilde{\Psi}: \mathcal{C C}(\omega) / \approx \rightarrow \mathcal{C C}\left(\bigcup_{s \in \mathbb{R}^{+}} \phi_{-s}(\omega)\right) / \sim
$$

In particular, the numbers of equivalence classes for $\approx$ in $\mathcal{C C}(\omega)$ and for $\sim$ in $\mathcal{C C}\left(\bigcup_{s \in \mathbb{R}^{+}} \phi_{-s}(\omega)\right)$ are equal.

The proof of Lemma 3.1 is given in Appendix 3.1. As a consequence of this lemma, all the results of this paper (together with their proofs) can be formulated with $\approx$ or with $\sim$ equivalently.

\subsection{Comparing $C^{-}(\infty)>0$ and GCC}

The statement that $C^{-}(\infty)>0$ is in general stronger than the fact that $\omega$ satisfies the Geometric Control Condition with respect to the hamiltonian $H(x, v)=\frac{|v|^{2}}{2}+V(x)$. This is due to the the non-compactness of the phase space $\mathbb{T}^{d} \times \mathbb{R}^{d}$.

Assume for instance that $V=0$ so that $\phi_{t}(x, v)=(x+t v, v)$ : the characteristic curves are straight lines and the velocity component $v$ is preserved by the flow. Take $k\left(x, v^{\prime}, v\right)>0$ on the whole $\mathbb{T}^{d} \times \mathbb{R}^{d} \times \mathbb{R}^{d}$. In this situation, $\omega=\mathbb{T}^{d} \times \mathbb{R}^{d}$ and so, it satisfies automatically GCC in any positive time. Assume further that $k$ does not depend on the space variable, i.e. $k\left(x, v^{\prime}, v\right)=k\left(v^{\prime}, v\right)$ and that there exists a sequence $\left(v_{n}\right)$ such that $\int_{\mathbb{R}^{d}} k\left(v_{n}, v^{\prime}\right) d v^{\prime} \rightarrow 0$. Then, we have $\left(\int_{\mathbb{R}^{d}} k\left(\cdot, v^{\prime}\right) d v^{\prime}\right)$ 。 $\phi_{t}(x, v)=\int_{\mathbb{R}^{d}} k\left(v, v^{\prime}\right) d v^{\prime}$ (as the flow preserves $v$ ) and hence, for any $n \in \mathbb{N}$, we 
have $C^{-}(\infty) \leq \int_{\mathbb{R}^{d}} k\left(v_{n}, v^{\prime}\right) d v^{\prime}$. This yields $C^{-}(\infty)=0$ although $\omega$ satisfies GCC. As an explicit example, one can take $k\left(x, v^{\prime}, v\right)=\mathcal{M}\left(v^{\prime}\right) \mathcal{M}(v)^{2}$.

Note finally that if $\int_{\mathbb{R}^{d}} k\left(x, v, v^{\prime}\right) d v^{\prime}$ is uniformly bounded from below at infinity (i.e. there exists $C, R>0$ such that $\int_{\mathbb{R}^{d}} k\left(x, v, v^{\prime}\right) d v^{\prime} \geq C$ for all $(x, v) \in \mathbb{T}^{d} \times$ $\left.B(0, R)^{c}\right)$, then $C^{-}(\infty)>0$ and GCC become equivalent.

The next paragraph shows that our assumption is indeed more general.

\subsection{Example of Exponential Convergence Without a Bound from Below at Infinity}

Here, we produce a simple example of dynamics and collision kernel such that $C^{-}(\infty)>0$, but neither $\tilde{k}$ nor $\int_{\mathbb{R}^{d}} k\left(x, v, v^{\prime}\right) d v^{\prime}$ are uniformly bounded from below at infinity.

For this, assume $(x, v) \in \mathbb{T} \times \mathbb{R}$ (we could construct similar examples in higher dimensions as well) and take $V=0$, so that $\phi_{t}(x, v)=(x+t v, v)$. We identify $\mathbb{T}$ to $[-1 / 2,1 / 2)$ with periodic boundary conditions. Define $\alpha \in C^{0}\left(\mathbb{T} ; \mathbb{R}^{+}\right)$with support contained in $(-1 / 3,1 / 3)$ and satisfying $\alpha=1$ on $[-1 / 4,1 / 4]$ and $\psi \in C^{0}\left(\mathbb{R} ; \mathbb{R}^{+}\right)$ such that $\psi(v) \rightarrow|v| \rightarrow+\infty 0, \psi>0$ and $\psi=1$ on $[-2,2]$. Consider the collision kernel in the class E1

$$
k\left(x, v, v^{\prime}\right)=\tilde{k}\left(x, v, v^{\prime}\right) \mathcal{M}\left(v^{\prime}\right), \quad \tilde{k}\left(x, v, v^{\prime}\right)=\left[\alpha(x)+\psi(v) \psi\left(v^{\prime}\right)\right] .
$$

We first readily check that $\tilde{k}>0$ on $\mathbb{T} \times \mathbb{R} \times \mathbb{R}$ and hence $\omega=\mathbb{T} \times \mathbb{R}$. We also remark that for any $R>0$,

$\inf _{\left(x, v, v^{\prime}\right) \in \mathbb{T} \times B(0, R)^{c} \times B(0, R)^{c}} \tilde{k}\left(x, v, v^{\prime}\right)=0, \quad$ and $\quad \inf _{(x, v) \in \mathbb{T} \times B(0, R)^{c}} \int_{\mathbb{R}} k\left(x, v, v^{\prime}\right) d v^{\prime}=0$.

Nevertheless, we can prove that $C^{-}(\infty) \geq C^{-}(1)>0$, and thus, by Theorem 2.3, there is exponential convergence to the Maxwellian equilibrium. We set $\beta:=\int_{\mathbb{R}} \psi\left(v^{\prime}\right) \mathcal{M}\left(v^{\prime}\right) d v^{\prime}>0$ and take $(x, v) \in \mathbb{T} \times \mathbb{R}$.

- If $v \in[-2,2]$, then we have $k\left(\phi_{t}(x, v), v^{\prime}\right)=k\left(x+t v, v, v^{\prime}\right) \geq \psi(v) \psi\left(v^{\prime}\right) \mathcal{M}\left(v^{\prime}\right)$ so that

$$
\int_{0}^{1} \int_{\mathbb{R}} k\left(\phi_{t}(x, v), v^{\prime}\right) d v^{\prime} d t \geq \beta \int_{0}^{1} \psi(v) d t=\beta>0 .
$$

- If $v \notin[-2,2]$, then, denoting by $\lfloor v\rfloor$ the integer part of $v$, we have

$$
\begin{aligned}
\int_{0}^{1} \int_{\mathbb{R}} k\left(\phi_{t}(x, v), v^{\prime}\right) d v^{\prime} d t & \geq \int_{0}^{1} \alpha(x+t v) d t \geq \int_{0}^{\frac{\lfloor v\rfloor}{|v|}} \alpha(x+t v) d t \\
& \geq \frac{1}{2} \frac{\lfloor v\rfloor}{|v|} \geq \frac{1}{4}
\end{aligned}
$$

This proves that $C^{-}(1)>0$ and thus $C^{-}(\infty)>0$. 


\section{Preliminary Results}

\subsection{Well-Posedness and Dissipation}

For readability, we shall sometimes denote

$$
\mathscr{C}(f):=\mathscr{C}(f)(x, v)=\int_{\mathbb{R}^{d}}\left[k\left(x, v^{\prime}, v\right) f\left(v^{\prime}\right)-k\left(x, v, v^{\prime}\right) f(v)\right] d v^{\prime} .
$$

The following dissipation identity holds for sufficiently smooth and decaying solutions to (1.1).

Lemma 4.1 Let $k$ be collision kernel satisfying A1-A3. Let $f$ be a smooth and sufficiently decaying (at infinity in the variable v) solution to (1.1). The following identity holds, for all $t \in \mathbb{R}^{+}$:

$$
\frac{d}{d t}\|f(t)\|_{\mathcal{L}^{2}}^{2}=-\mathscr{D}(f)
$$

where $\mathscr{D}(f)=-2\langle\mathscr{C}(f), f\rangle_{\mathcal{L}^{2}}$ satisfies

$$
\begin{aligned}
\mathscr{D}(f)= & \frac{1}{2} \int_{\mathbb{T}^{d}} e^{V} \int_{\mathbb{R}^{d}} \int_{\mathbb{R}^{d}}\left(\frac{k\left(x, v^{\prime}, v\right)}{\mathcal{M}(v)}+\frac{k\left(x, v, v^{\prime}\right)}{\mathcal{M}\left(v^{\prime}\right)}\right) \mathcal{M}(v) \mathcal{M}\left(v^{\prime}\right) \\
& \times\left(\frac{f(t, x, v)}{\mathcal{M}(v)}-\frac{f\left(t, x, v^{\prime}\right)}{\mathcal{M}\left(v^{\prime}\right)}\right)^{2} d v^{\prime} d v d x
\end{aligned}
$$

The term $\mathscr{D}(f)$ will often be referred to as the dissipation term in the following. The proof is rather classical and follows [15].

Proof of Lemma 4.1 Multiply (1.1) by $f \frac{e^{V}}{\mathcal{M}(v)}$ and integrate with respect to $x$ and $v$. This yields

$$
\begin{aligned}
& \frac{1}{2} \frac{d}{d t}\|f(t)\|_{\mathcal{L}^{2}}^{2}+\int_{\mathbb{T}^{d} \times \mathbb{R}^{d}}\left(v \cdot \nabla_{x} f-\nabla_{x} V \cdot \nabla_{v} f\right) f \frac{e^{V}}{\mathcal{M}(v)} d v d x \\
& \quad=\int_{\mathbb{T}^{d}} e^{V} \int_{\mathbb{R}^{d}} \mathscr{C}(f) \frac{f}{\mathcal{M}} d v d x .
\end{aligned}
$$

On the one hand, the contribution of the transport term vanishes

$$
\begin{aligned}
& \int_{\mathbb{T}^{d} \times \mathbb{R}^{d}}\left(v \cdot \nabla_{x} f-\nabla_{x} V \cdot \nabla_{v} f\right) f \frac{e^{V}}{\mathcal{M}(v)} d v d x \\
& =\frac{1}{2} \int_{\mathbb{T}^{d} \times \mathbb{R}^{d}}\left(v \cdot \nabla_{x}-\nabla_{x} V \cdot \nabla_{v}\right)|f|^{2} \frac{e^{V}}{\mathcal{M}(v)} d v d x \\
& =-\frac{1}{2} \int_{\mathbb{T}^{d} \times \mathbb{R}^{d}}|f|^{2}\left(v \cdot \nabla_{x}-\nabla_{x} V \cdot \nabla_{v}\right) \frac{e^{V}}{\mathcal{M}(v)} d v d x \\
& =0
\end{aligned}
$$


since $\left(v \cdot \nabla_{x}-\nabla_{x} V \cdot \nabla_{v}\right) \frac{e^{V}}{\mathcal{M}(v)}=0$. On the other hand, following [15], we have for any $x \in \mathbb{T}^{d}$ the identity

$$
\begin{array}{rl}
\int_{\mathbb{R}^{d}} & \mathscr{C}(f)(x, v) \frac{f}{\mathcal{M}} d v \\
= & \int_{\mathbb{R}^{d}} \int_{\mathbb{R}^{d}}\left[k\left(x, v^{\prime}, v\right) f\left(v^{\prime}\right)-k\left(x, v, v^{\prime}\right) f(v)\right] d v^{\prime} \frac{f(v)}{\mathcal{M}(v)} d v \\
= & \int_{\mathbb{R}^{d}} \int_{\mathbb{R}^{d}} k\left(x, v^{\prime}, v\right) f\left(v^{\prime}\right) \frac{f(v)}{\mathcal{M}(v)} d v^{\prime} d v-\int_{\mathbb{R}^{d}} \int_{\mathbb{R}^{d}} k\left(x, v, v^{\prime}\right) \frac{|f(v)|^{2}}{\mathcal{M}(v)} d v^{\prime} d v .
\end{array}
$$

Symmetrizing the first term in the right hand-side of (4.3) yields

$$
\begin{aligned}
\int_{\mathbb{R}^{d}} & \int_{\mathbb{R}^{d}} k\left(x, v^{\prime}, v\right) f\left(v^{\prime}\right) \frac{f(v)}{\mathcal{M}(v)} d v^{\prime} d v \\
= & \frac{1}{2} \int_{\mathbb{R}^{d}} \int_{\mathbb{R}^{d}} k\left(x, v^{\prime}, v\right) f\left(v^{\prime}\right) \frac{f(v)}{\mathcal{M}(v)} d v^{\prime} d v \\
& +\frac{1}{2} \int_{\mathbb{R}^{d}} \int_{\mathbb{R}^{d}} k\left(x, v, v^{\prime}\right) f(v) \frac{f\left(v^{\prime}\right)}{\mathcal{M}\left(v^{\prime}\right)} d v^{\prime} d v
\end{aligned}
$$

Concerning the second term in the right hand-side of (4.3), we use (1.4) to obtain

$$
\begin{aligned}
- & \int_{\mathbb{R}^{d}} \int_{\mathbb{R}^{d}} k\left(x, v, v^{\prime}\right) \frac{|f(v)|^{2}}{\mathcal{M}(v)} d v^{\prime} d v \\
= & -\frac{1}{2} \int_{\mathbb{R}^{d}} \int_{\mathbb{R}^{d}} k\left(x, v, v^{\prime}\right) \frac{|f(v)|^{2}}{\mathcal{M}(v)} d v^{\prime} d v \\
& -\frac{1}{2} \int_{\mathbb{R}^{d}} \int_{\mathbb{R}^{d}} k\left(x, v, v^{\prime}\right) \mathcal{M}(v) \frac{|f(v)|^{2}}{\mathcal{M}(v)^{2}} d v^{\prime} d v \\
= & -\frac{1}{2} \int_{\mathbb{R}^{d}} \int_{\mathbb{R}^{d}} k\left(x, v, v^{\prime}\right) \frac{|f(v)|^{2}}{\mathcal{M}(v)} d v^{\prime} d v \\
& -\frac{1}{2} \int_{\mathbb{R}^{d}} \int_{\mathbb{R}^{d}} k\left(x, v^{\prime}, v\right) \mathcal{M}\left(v^{\prime}\right) \frac{|f(v)|^{2}}{\mathcal{M}(v)^{2}} d v^{\prime} d v \\
= & -\frac{1}{4} \int_{\mathbb{R}^{d}} \int_{\mathbb{R}^{d}} k\left(x, v, v^{\prime}\right) \frac{|f(v)|^{2}}{\mathcal{M}(v)} d v^{\prime} d v-\frac{1}{4} \int_{\mathbb{R}^{d}} \int_{\mathbb{R}^{d}} k\left(x, v^{\prime}, v\right) \frac{\left|f\left(v^{\prime}\right)\right|^{2}}{\mathcal{M}\left(v^{\prime}\right)} d v^{\prime} d v \\
& -\frac{1}{4} \int_{\mathbb{R}^{d}} \int_{\mathbb{R}^{d}} k\left(x, v^{\prime}, v\right) \mathcal{M}\left(v^{\prime}\right) \frac{|f(v)|^{2}}{\mathcal{M}(v)^{2}} d v^{\prime} d v \\
& -\frac{1}{4} \int_{\mathbb{R}^{d}} \int_{\mathbb{R}^{d}} k\left(x, v, v^{\prime}\right) \mathcal{M}(v) \frac{\left|f\left(v^{\prime}\right)\right|^{2}}{\mathcal{M}\left(v^{\prime}\right)^{2}} d v^{\prime} d v .
\end{aligned}
$$

Combining the last two identities, we can now collect together the terms with $k\left(x, v^{\prime}, v\right)$ (resp. $k\left(x, v, v^{\prime}\right)$ ) and rewrite the right hand-side of (4.3) as a sum of two squares. Namely, this provides 


$$
\begin{aligned}
\int_{\mathbb{R}^{d}} \mathscr{C}(f)(x, v) \frac{f}{\mathcal{M}} d v= & -\frac{1}{4} \int_{\mathbb{R}^{d}} \int_{\mathbb{R}^{d}}\left(\frac{k\left(x, v^{\prime}, v\right)}{\mathcal{M}(v)}+\frac{k\left(x, v, v^{\prime}\right)}{\mathcal{M}\left(v^{\prime}\right)}\right) \mathcal{M}(v) \mathcal{M}\left(v^{\prime}\right) \\
& \times\left(\frac{f(v)}{\mathcal{M}(v)}-\frac{f\left(v^{\prime}\right)}{\mathcal{M}\left(v^{\prime}\right)}\right)^{2} d v^{\prime} d v
\end{aligned}
$$

This yields (4.1) and concludes the proof of the Lemma.

We have the following useful lemma for the dissipation functional $\mathscr{D}$.

Lemma 4.2 Assume that $\left(h_{n}\right)$ is a sequence of measurable functions such that

$$
\sup _{n} \int_{0}^{T} \mathscr{D}\left(h_{n}(t)\right) d t<+\infty
$$

and such that $h_{n} \rightarrow h$ in the sense of distributions. Then, we have

$$
\int_{0}^{T} \mathscr{D}(h(t)) d t \leq \liminf _{n \rightarrow+\infty} \int_{0}^{T} \mathscr{D}\left(h_{n}(t)\right) d t .
$$

Proof of Lemma 4.2 We denote

$$
d \lambda:=\mathbb{1}_{[0, T]}(t)\left(\frac{k\left(x, v^{\prime}, v\right)}{\mathcal{M}(v)}+\frac{k\left(x, v, v^{\prime}\right)}{\mathcal{M}\left(v^{\prime}\right)}\right) \mathcal{M}(v) \mathcal{M}\left(v^{\prime}\right) d v d v^{\prime} d x d t .
$$

Then, introducing

$$
\tilde{h}_{n}\left(t, x, v, v^{\prime}\right):=\frac{h_{n}(t, x, v)}{\mathcal{M}(v)}-\frac{h_{n}\left(t, x, v^{\prime}\right)}{\mathcal{M}\left(v^{\prime}\right)},
$$

we observe that $\left\|\tilde{h}_{n}\right\|_{L^{2}(d \lambda)}^{2}=\int_{0}^{T} \mathscr{D}\left(h_{n}\right) d t$ and thus, by (4.4), we deduce that $\tilde{h}_{n}\left(t, x, v, v^{\prime}\right)$ is uniformly bounded in $L^{2}(d \lambda)$. Consequently, up to extracting a subsequence, $\tilde{h}_{n}$ weakly converges in $L^{2}(d \lambda)$ to some function $\tilde{h}$. By uniqueness of the limit in the sense of distributions, we have

$$
\tilde{h}=\frac{h(t, x, v)}{\mathcal{M}(v)}-\frac{h\left(t, x, v^{\prime}\right)}{\mathcal{M}\left(v^{\prime}\right)} .
$$

Then, by (4.4) and weak lower semi-continuity, we deduce that for any $T>0$, we have

$$
\|\mathscr{D}(h)\|_{L^{1}(0, T)}=\|\tilde{h}\|_{L^{2}(d \lambda)}^{2} \leq \liminf _{n \rightarrow+\infty}\left\|\tilde{h}_{n}\right\|_{L^{2}(d \lambda)}^{2}=\liminf _{n \rightarrow+\infty}\left\|\mathscr{D}\left(h_{n}\right)\right\|_{L^{1}(0, T)} .
$$

We can now state the main well-posedness result (which uses all assumptions A1A2-A3). 
Proposition 4.1 (Well-posedness of the linear Boltzmann equation). Assume that $f_{0} \in \mathcal{L}^{2}$. Then there exists a unique solution $f \in C^{0}\left(\mathbb{R}^{+} ; \mathcal{L}^{2}\right)$ of (1.1) satisfying $\left.f\right|_{t=0}=f_{0}$. Moreover, the solution $f$ satisfies

$$
\begin{gathered}
\int_{0}^{+\infty} \mathscr{D}(f(s)) d s \leq\left\|f_{0}\right\|_{\mathcal{L}^{2}}^{2}<+\infty \\
\int_{0}^{t} \int_{\mathbb{T}^{d} \times \mathbb{R}^{d}}\left(\int_{\mathbb{R}^{d}} k\left(x, v, v^{\prime}\right) d v^{\prime}\right) \frac{|f(s, x, v)|^{2} e^{V(x)}}{\mathcal{M}(v)} d v d x d s<+\infty, \quad \text { for all } t \geq 0,
\end{gathered}
$$

and we have

$$
\text { for all } 0 \leq t^{\prime} \leq t, \quad\|f(t)\|_{\mathcal{L}^{2}}^{2}-\left\|f\left(t^{\prime}\right)\right\|_{\mathcal{L}^{2}}^{2}=-\int_{t^{\prime}}^{t} \mathscr{D}(f(s)) d s,
$$

where $\mathscr{D}(f)$ is defined in (4.2). If moreover $f_{0} \geq 0$ a.e., then for all $t \in \mathbb{R}$ we have $f(t, \cdot, \cdot) \geq 0$ a.e. (Maximum principle).

Note that in the case where $\int_{\mathbb{R}^{d}} k\left(x, v, v^{\prime}\right) d v^{\prime}$ is not a bounded function (in the variable $v \in \mathbb{R}^{d}$ ), then this proposition in particular states a gain of integrability of solutions of (1.1).

Let us start with a preliminary description of the operators (on the space $\mathcal{L}^{2}$ ) that are involved. We write

$$
\begin{aligned}
\left(A_{0} f\right)(x, v) & =\left(v \cdot \nabla_{x}-\nabla_{x} V \cdot \nabla_{v}\right) f(x, v)+\left(\int_{\mathbb{R}^{d}} k\left(x, v, v^{\prime}\right) d v^{\prime}\right) f(x, v), \\
(K f)(x, v) & =-\int_{\mathbb{R}^{d}} k\left(x, v^{\prime}, v\right) f\left(x, v^{\prime}\right) d v^{\prime} \\
A f & =A_{0} f+K f .
\end{aligned}
$$

The domain of $A_{0}$ is given by

$$
D\left(A_{0}\right)=\left\{f \in \mathcal{L}^{2},\left(v \cdot \nabla_{x}-\nabla_{x} V \cdot \nabla_{v}\right) f+\left(\int_{\mathbb{R}^{d}} k\left(\cdot, v^{\prime}\right) d v^{\prime}\right) f \in \mathcal{L}^{2}\right\} .
$$

The following lemma describes the very first properties enjoyed by functions of $D\left(A_{0}\right)$.

Lemma 4.3 For all $f \in D\left(A_{0}\right)$, we have

$$
\int_{\mathbb{T}^{d} \times \mathbb{R}^{d}}\left(\int_{\mathbb{R}^{d}} k\left(x, v, v^{\prime}\right) d v^{\prime}\right)|f(x, v)|^{2} \frac{e^{V(x)}}{\mathcal{M}(v)} d x d v \leq\left\|A_{0} f\right\|_{\mathcal{L}^{2}}\|f\|_{\mathcal{L}^{2}}<+\infty
$$

and

$$
\left\langle\left(v \cdot \nabla_{x}-\nabla_{x} V \cdot \nabla_{v}\right) f, f\right\rangle_{\mathcal{L}^{2}}=0 .
$$


Proof of Lemma 4.3 Let $\Psi \in C_{c}^{\infty}(\mathbb{R},[0,1])$, with $\Psi \equiv 1$ on $[-1,1]$ and $\Psi \equiv 0$ on $(-\infty, 2) \cup(2,+\infty)$. Let $f \in D\left(A_{0}\right)$. We set $\Psi_{n}(x, v):=\Psi\left(\frac{H(x, v)}{n}\right)$, where we recall that $H$ is the hamiltonian. Hence, $\Psi_{n}$ is compactly supported. Note also that we have

$$
\left(v \cdot \nabla_{x}-\nabla_{x} V \cdot \nabla_{v}\right) \Psi_{n}=0 .
$$

We define the compactly supported functions $f_{n}:=\Psi_{n} f$. Using a dominated convergence argument, we have

$$
\left\|f_{n}-f\right\|_{\mathcal{L}^{2}} \rightarrow 0, \quad\left\|A_{0} f_{n}-A_{0} f\right\|_{\mathcal{L}^{2}} \rightarrow 0
$$

noticing that $A_{0} f_{n}=\Psi_{n} A_{0} f$. We deduce that

$$
\left\langle A_{0} f_{n}, f_{n}\right\rangle_{\mathcal{L}^{2}} \rightarrow\left\langle A_{0} f, f\right\rangle_{\mathcal{L}^{2}}
$$

Furthermore, since $f \in D\left(A_{0}\right)$, we have $\left(v \cdot \nabla_{x}-\nabla_{x} V \cdot \nabla_{v}\right) f \in L_{l o c}^{2}$ and thus

$$
\left(v \cdot \nabla_{x}-\nabla_{x} V \cdot \nabla_{v}\right) f_{n} \in \mathcal{L}^{2} .
$$

We can hence compute (using a classical density argument)

$$
\begin{aligned}
\left\langle\left(v \cdot \nabla_{x}-\nabla_{x} V \cdot \nabla_{v}\right) f_{n}, f_{n}\right\rangle_{\mathcal{L}^{2}} & =\left\langle\left(v \cdot \nabla_{x}-\nabla_{x} V \cdot \nabla_{v}\right) \frac{\left|f_{n}\right|^{2}}{2}, \Psi_{n}\right\rangle_{\mathcal{L}^{2}} \\
& =\left\langle\frac{\left|f_{n}\right|^{2}}{2},\left(v \cdot \nabla_{x}-\nabla_{x} V \cdot \nabla_{v}\right) \Psi_{n}\right\rangle_{\mathcal{L}^{2}} \\
& =0 .
\end{aligned}
$$

Therefore, we deduce

$$
\left\langle A_{0} f_{n}, f_{n}\right\rangle_{\mathcal{L}^{2}}=\int_{\mathbb{T}^{d} \times \mathbb{R}^{d}}\left(\int_{\mathbb{R}^{d}} k\left(x, v, v^{\prime}\right) d v^{\prime}\right)\left|f_{n}(x, v)\right|^{2} \frac{e^{V(x)}}{\mathcal{M}(v)} d x d v,
$$

and thus by a weak lower semi-continuity argument, that

$$
\int_{\mathbb{T}^{d} \times \mathbb{R}^{d}}\left(\int_{\mathbb{R}^{d}} k\left(x, v, v^{\prime}\right) d v^{\prime}\right)|f(x, v)|^{2} \frac{e^{V(x)}}{\mathcal{M}(v)} d x d v \leq C\left\|A_{0} f\right\|_{\mathcal{L}^{2}}\|f\|_{\mathcal{L}^{2}}
$$

We can therefore finally pass to the limit in the identity

$$
\left\langle A_{0} f_{n}, f_{n}\right\rangle_{\mathcal{L}^{2}}-\left\langle\left(\int_{\mathbb{R}^{d}} k\left(\cdot, v^{\prime}\right) d v^{\prime}\right) f_{n}, f_{n}\right\rangle_{\mathcal{L}^{2}}=0
$$


to infer that

$$
\left\langle A_{0} f, f\right\rangle_{\mathcal{L}^{2}}-\left\langle\left(\int_{\mathbb{R}^{d}} k\left(\cdot, v^{\prime}\right) d v^{\prime}\right) f, f\right\rangle_{\mathcal{L}^{2}}=0
$$

that is to say

$$
\left\langle\left(v \cdot \nabla_{x}-\nabla_{x} V \cdot \nabla_{v}\right) f, f\right\rangle_{\mathcal{L}^{2}}=0 .
$$

This concludes the proof of the lemma.

Lemma 4.4 The operator $K$ is bounded on $\mathcal{L}^{2}$ and $D(A)=D\left(A_{0}\right)$. The Boltzmann operator A generates a strongly continuous semigroup $e^{-t A}$ on $\mathcal{L}^{2}$ and we have the Duhamel type formula

$$
e^{-t A}=e^{-t A_{0}}+\int_{0}^{t} e^{-(t-s) A_{0}} K e^{-s A} d s
$$

Note that this only uses A1 and A3.

Proof of Lemma 4.4 On the one hand, we have the explicit formula, for $t \geq 0$,

$$
e^{-t A_{0}} u(x, v)=\exp \left(-\int_{0}^{t} \int_{\mathbb{R}^{d}} k\left(\phi_{-(t-s)}(x, v), v^{\prime}\right) d v^{\prime} d s\right) u \circ \phi_{-t}(x, v),
$$

where $\phi_{s}(x, v)=\left(X_{s}(x, v), \Xi_{s}(x, v)\right)$ denotes the hamiltonian flow of Definition 2.2. Using $k \geq 0$ and remarking that the change of variables $\phi_{-t}(x, v) \rightarrow(x, v)$ preserves $\frac{e^{V}}{\mathcal{M}}$ and has unit Jacobian, we obtain

$$
\left\|e^{-t A_{0}} u\right\|_{\mathcal{L}^{2}} \leq\|u\|_{\mathcal{L}^{2}}, \quad \text { for } t \geq 0 .
$$

Moreover, for $u \in C_{c}^{\infty}\left(\mathbb{T}^{d} \times \mathbb{R}^{d}\right)$, with $S=\operatorname{supp}(u)$, we have

$$
\begin{aligned}
& \left\|e^{-t A_{0}} u-u\right\|_{\mathcal{L}^{2}}^{2} \\
& \quad=\int_{K}\left|e^{\left(-\int_{0}^{t} \int_{\mathbb{R}^{d}} k\left(\phi_{-(t-s)}(x, v), v^{\prime}\right) d v^{\prime} d s\right)} u \circ \phi_{-t}(x, v)-u(x, v)\right|^{2} \frac{e^{V(x)}}{\mathcal{M}(v)} d x d v,
\end{aligned}
$$

where $K=S \cup \phi_{t}(S)$. The regularity of the flow $\phi_{t}$ yields $\phi_{-t}(x, v)=(x, v)+$ $O(t)$ where $O(t)$ is uniform on the compact set $S$ as $t \rightarrow 0^{+}$. Therefore, using the smoothness of $u$, a Taylor expansion proves that $\lim _{t \rightarrow 0^{+}}\left\|e^{-t A_{0}} u-u\right\|_{\mathcal{L}^{2}}=0$.

Next, take any $u \in \mathcal{L}^{2}$ and fix $\varepsilon>0$. There is $u^{\varepsilon} \in C_{c}^{\infty}\left(\mathbb{T}^{d} \times \mathbb{R}^{d}\right)$ such that $\left\|u-u^{\varepsilon}\right\|_{\mathcal{L}^{2}}<\varepsilon / 3$, and according to (4.7), $\left\|e^{-t A_{0}}\left(u-u^{\varepsilon}\right)\right\|_{\mathcal{L}^{2}} \leq\left\|u-u^{\varepsilon}\right\|_{\mathcal{L}^{2}}<\varepsilon / 3$. We also have $\left\|e^{-t A_{0}} u^{\varepsilon}-u^{\varepsilon}\right\|_{\mathcal{L}^{2}}^{2} \rightarrow 0$ as $t \rightarrow 0^{+}$and thus, there exists $\delta>0$ such that $\left\|e^{-t A_{0}} u^{\varepsilon}-u^{\varepsilon}\right\|_{\mathcal{L}^{2}}<\varepsilon / 3$ for $0<t<\delta$. Finally, we have for $0<t<\delta$

$$
\left\|e^{-t A_{0}} u-u\right\|_{\mathcal{L}^{2}} \leq\left\|e^{-t A_{0}}\left(u-u^{\varepsilon}\right)\right\|_{\mathcal{L}^{2}}+\left\|u-u^{\varepsilon}\right\|_{\mathcal{L}^{2}}+\left\|e^{-t A_{0}} u^{\varepsilon}-u^{\varepsilon}\right\|_{\mathcal{L}^{2}}<\varepsilon
$$


This means that $\lim _{t \rightarrow 0^{+}}\left\|e^{-t A_{0}} u-u\right\|_{\mathcal{L}^{2}}=0$ so that the operator $A_{0}$ generates a strongly continuous semigroup of contraction on $\mathcal{L}^{2}$, explicited by (4.6).

On the other hand, using the assumption $\mathbf{A 3}$, the operator $K$ is bounded on $\mathcal{L}^{2}$. Indeed, by the Cauchy-Schwarz inequality, we have

$$
\begin{aligned}
\|K f\|_{\mathcal{L}^{2}}^{2} & =\int_{\mathbb{T}^{d} \times \mathbb{R}^{d}}\left(\int_{\mathbb{R}^{d}} k\left(x, v^{\prime}, v\right) f\left(x, v^{\prime}\right) d v^{\prime}\right)^{2} \frac{e^{V(x)}}{\mathcal{M}(v)} d x d v \\
& \leq \int_{\mathbb{T}^{d} \times \mathbb{R}^{d}} \frac{e^{V(x)}}{\mathcal{M}(v)}\left(\int_{\mathbb{R}^{d}} k\left(x, v^{\prime}, v\right)^{2} \mathcal{M}\left(v^{\prime}\right) d v^{\prime}\right)\left(\int_{\mathbb{R}^{d}} \frac{f\left(x, v^{\prime}\right)^{2}}{\mathcal{M}\left(v^{\prime}\right)} d v^{\prime}\right) d x d v \\
& \left.\leq \sup _{x \in \mathbb{T}^{d}} \int_{\mathbb{R}^{d}} \int_{\mathbb{R}^{d}} k^{2}\left(x, v^{\prime}, v\right) \frac{\mathcal{M}\left(v^{\prime}\right)}{\mathcal{M}(v)} d v^{\prime} d v\right)\|f\|_{\mathcal{L}^{2}}^{2} .
\end{aligned}
$$

The operator $K$ is hence bounded in $\mathcal{L}^{2}$, with

$$
\|K\|_{\mathcal{L}^{2} \rightarrow \mathcal{L}^{2}} \leq\left(\sup _{x \in \mathbb{T}^{d}} \int_{\mathbb{R}^{d}} \int_{\mathbb{R}^{d}} k^{2}\left(x, v^{\prime}, v\right) \frac{\mathcal{M}\left(v^{\prime}\right)}{\mathcal{M}(v)} d v^{\prime} d v\right)^{\frac{1}{2}}
$$

Therefore $D(A)=D\left(A_{0}\right)$ and according to [36, Chapter 3, Theorem 1.1], $A$ therefore generates a strongly continuous semigroup $e^{-t A}$ on $\mathcal{L}^{2}$. The Duhamel formula also follows from the boundedness of $K$.

Proof of Proposition 4.1 Lemma 4.4 implies the existence of a unique solution $f(t) \in$ $C^{0}\left(\mathbb{R}^{+} ; \mathcal{L}^{2}\right)$ to $(1.1)$ with initial condition $f_{0}$. For what concerns the maximum principle, we can argue as follows. Assume that $f_{0} \geq 0$ a.e. We may denote by $C^{0}\left(0, T ; \mathcal{L}^{2}\right)_{+}$ the subset of $C^{0}\left(0, T ; \mathcal{L}^{2}\right)$ of almost everywhere nonegative functions, and define the map

$$
\begin{aligned}
J_{+}: C^{0}\left(0, T ; \mathcal{L}^{2}\right)_{+} & \rightarrow C^{0}\left(0, T ; \mathcal{L}^{2}\right)_{+} \\
\varphi & \mapsto e^{-t A_{0}} f_{0}+\int_{0}^{t} e^{-(t-s) A_{0}}(K \varphi(s, \cdot)) d s .
\end{aligned}
$$

We have the contraction estimate

$$
\left\|J\left(\varphi_{1}\right)-J\left(\varphi_{2}\right)\right\|_{L^{\infty}\left(0, T ; \mathcal{L}^{2}\right)} \leq T\|K\|_{\mathcal{L}^{2} \rightarrow \mathcal{L}^{2}}\left\|\varphi_{1}-\varphi_{2}\right\|_{L^{\infty}\left(0, T ; \mathcal{L}^{2}\right)} .
$$

For $T>0$ small enough, $J$ is contracting in the Banach space $C^{0}\left(0, T ; \mathcal{L}^{2}\right)_{+}$, and therefore admits a unique fixed point in $C^{0}\left(0, T ; \mathcal{L}^{2}\right)_{+}$, which is $f$ by uniqueness of the solution to (1.1). Thus, $f \geq 0$ a.e. on $[0, T]$, and then on $\mathbb{R}^{+}$by the time translation invariance of (1.1).

Let us finally prove the dissipation identity (4.5). We rely on Lemma 4.1 and an approximation argument.

Note first that if $f_{0} \in D(A)$, it follows from [36, Chapter 1 , Theorem $\left.2.4 \mathrm{c}\right)$ ] that the associated solution $f$ satisfies $f \in C^{1}\left(\mathbb{R}^{+} ; \mathcal{L}^{2}\right) \cap C^{0}\left(\mathbb{R}^{+} ; D(A)\right)$, and, in particular, for all $t \geq 0, f(t) \in D(A)$. This entails that for all $t \geq 0$, we have 


$$
\begin{aligned}
& \int_{\mathbb{T}^{d} \times \mathbb{R}^{d}}\left(\int_{\mathbb{R}^{d}} k\left(x, v, v^{\prime}\right) d v^{\prime}\right)|f(t, x, v)|^{2} \frac{e^{V(x)}}{\mathcal{M}(v)} d x d v<+\infty, \\
& \left\langle\left(v \cdot \nabla_{x}-\nabla_{x} V \cdot \nabla_{v}\right) f(t), f(t)\right\rangle_{\mathcal{L}^{2}}=0 .
\end{aligned}
$$

For $f \in C^{0}\left(\mathbb{R}^{+} ; D(A)\right)$, the quantity $\mathscr{D}(f(t))$ is well defined for every $t$, remarking that (1.4) gives $\int_{\mathbb{R}^{d}} k\left(x, v^{\prime}, v\right) \frac{\mathcal{M}\left(v^{\prime}\right)}{\mathcal{M}(v)} d v^{\prime}=\int_{\mathbb{R}^{d}} k\left(x, v, v^{\prime}\right) d v^{\prime}$.

Moreover, for $f \in C^{1}\left(\mathbb{R}^{+} ; \mathcal{L}^{2}\right) \cap C^{0}\left(\mathbb{R}^{+} ; D(A)\right)$ all computations of the proof of Lemma 4.1 can be performed, so that for any $f_{0} \in D(A)$, we have

$$
\int_{0}^{t} \mathscr{D}(f(s)) d s=\left\|f_{0}\right\|_{\mathcal{L}^{2}}^{2}-\|f(t)\|_{\mathcal{L}^{2}}^{2} \quad \text { for all } t \geq 0 .
$$

Then, note that $C_{c}^{\infty}\left(\mathbb{T}^{d} \times \mathbb{R}^{d}\right) \subset D(A) \subset \mathcal{L}^{2}$, so that $D(A)$ is dense in $\mathcal{L}^{2}$. Let thus $\left(f_{0}^{n}\right)_{n>0}$ be a sequence such that $f_{0}^{n} \in D(A)$ and $\left\|f_{0}^{n}-f_{0}\right\|_{\mathcal{L}^{2}} \rightarrow 0$ as $n \rightarrow+\infty$. Denote by $f^{n}(t) \in C^{1}\left(\mathbb{R}^{+} ; \mathcal{L}^{2}\right) \cap C^{0}\left(\mathbb{R}^{+} ; D(A)\right)$ the solution of (1.1) with initial condition $f_{0}^{n}$ obtained from Lemma 4.4. We have in particular

$$
\left\|f^{n}-f\right\|_{L^{\infty}\left(0, t ; \mathcal{L}^{2}\right)} \rightarrow 0, \quad \text { for all } t \geq 0
$$

Since $f_{0}^{n} \in D(A)$, the dissipation identity (4.9) holds for $f^{n}$. From Lemma 4.2, we then deduce that for all $t \geq 0$,

$$
\begin{aligned}
\int_{0}^{t} \mathscr{D}(f) d s \leq \liminf _{n \rightarrow+\infty} \int_{0}^{t} \mathscr{D}\left(f^{n}\right) d s & =\liminf _{n \rightarrow+\infty}\left(\left\|f_{0}^{n}\right\|_{\mathcal{L}^{2}}^{2}-\left\|f^{n}(t)\right\|_{\mathcal{L}^{2}}^{2}\right) \\
& =\left\|f_{0}\right\|_{\mathcal{L}^{2}}^{2}-\|f(t)\|_{\mathcal{L}^{2}}^{2} .
\end{aligned}
$$

Using this information, we obtain from the definition of $\mathscr{D}(f)$ that

$$
\begin{aligned}
& \int_{0}^{t} \int_{\mathbb{T}^{d} \times \mathbb{R}^{d}}\left(\int_{\mathbb{R}^{d}} k\left(x, v, v^{\prime}\right) d v^{\prime}\right) \frac{|f(s, x, v)|^{2} e^{V(x)}}{\mathcal{M}(v)} d v d x d s \\
& \quad \leq \frac{1}{2} \int_{0}^{t} \mathscr{D}(f) d s+t\|K\|_{\mathcal{L}^{2} \rightarrow \mathcal{L}^{2}\|f\|_{L^{\infty}\left(0, t ; \mathcal{L}^{2}\right)}^{2}} \\
& \quad \leq \frac{1}{2}\left(\left\|f_{0}\right\|_{\mathcal{L}^{2}}^{2}-\|f(t)\|_{\mathcal{L}^{2}}^{2}\right)+t\|K\|_{\mathcal{L}^{2} \rightarrow \mathcal{L}^{2}\|f\|_{L^{\infty}\left(0, t ; \mathcal{L}^{2}\right)^{\prime}}^{2}}
\end{aligned}
$$

We now want to prove the dissipation identity for any solution $f \in C^{0}\left(\mathbb{R}^{+} ; \mathcal{L}^{2}\right)$. For this, introduce $\rho \in C_{c}^{\infty}(\mathbb{R})$ such that $\rho \geq 0, \int_{\mathbb{R}} \rho=1$ so that $\rho_{\delta}=\frac{1}{\delta} \rho\left(\frac{t}{\delta}\right)$ is an approximation of identity. We define $\bar{f}(t)$ such that $\bar{f}(t)=f(t)$ for $t \geq 0$ and $\bar{f}(t)=0$ for $t<0$, and set $f^{\delta}=\rho_{\delta} * \bar{f}$ where the convolution is only in the variable $t \in \mathbb{R}$. Since $f \in C^{0}\left(\mathbb{R}^{+} ; \mathcal{L}^{2}\right)$, we have (in particular) $f^{\delta} \in C^{1}\left(\mathbb{R}^{+} ; \mathcal{L}^{2}\right)$, and in the sense of distributions, we have

$$
\left(\partial_{t}+A\right) f^{\delta}=\rho_{\delta} *\left(\partial_{t}+A\right) f=0 \quad \text { on } \mathbb{R}_{*}^{+} \times \mathbb{T}^{d} \times \mathbb{R}^{d}
$$


Since $f^{\delta} \in C^{1}\left(\mathbb{R}^{+} ; \mathcal{L}^{2}\right)$, this equation yields $A f^{\delta}=-\partial_{t} f^{\delta} \in C^{0}\left(\mathbb{R}_{*}^{+} ; \mathcal{L}^{2}\right)$, so that $f^{\delta} \in C^{0}\left(\mathbb{R}_{*}^{+} ; D(A)\right)$. As a consequence, the following dissipation identity holds for $f^{\delta}$ :

$$
\int_{t^{\prime}}^{t} \mathscr{D}\left(f^{\delta}(s)\right) d s=\left\|f^{\delta}\left(t^{\prime}\right)\right\|_{\mathcal{L}^{2}}^{2}-\left\|f^{\delta}(t)\right\|_{\mathcal{L}^{2}}^{2} \quad \text { for all } t \geq t^{\prime}>0
$$

Next, the usual proofs for approximations of identity show that

- $\left\|f^{\delta}(t)-f(t)\right\|_{\mathcal{L}^{2}} \rightarrow 0$ for all $t>0$;

- If $f \in L^{2}\left(t^{\prime}, t ; \mathscr{L}^{2}\right)$, with $\mathscr{L}^{2}=L^{2}\left(\mathbb{T}^{d} \times \mathbb{R}^{d}, \Psi(x, v) d x d v\right)$ where $\Psi \geq 0$ is a measurable real valued function, then $f^{\delta} \in L^{2}\left(t^{\prime}, t ; \mathscr{L}^{2}\right)$ and we have $\| f^{\delta}-$ $f \|_{L^{2}\left(t^{\prime}, t ; \mathscr{L}^{2}\right)} \rightarrow 0$.

The first item allows to pass to the limit $\delta \rightarrow 0^{+}$in the right hand-side of (4.10).

Moreover, we have proved that the solution $f \in C^{0}\left(\mathbb{R}^{+} ; \mathcal{L}^{2}\right)$ is such that the two quantities $\int_{t^{\prime}}^{t} \mathscr{D}(f) d s$ and $\int_{t^{\prime}}^{t} \int_{\mathbb{T}^{d} \times \mathbb{R}^{d}}\left(\int_{\mathbb{R}^{d}} k\left(x, v, v^{\prime}\right) d v^{\prime}\right) \frac{|f(s, x, v)|^{2} e^{V(x)}}{\mathcal{M}(v)} d v d x d s$ are finite. In particular, we have

$$
\begin{aligned}
\int_{t^{\prime}}^{t} \mathscr{D}(f) d s= & \int_{t^{\prime}}^{t} \int_{\mathbb{T}^{d} \times \mathbb{R}^{d}}\left(\int_{\mathbb{R}^{d}} k\left(x, v, v^{\prime}\right) d v^{\prime}\right) \frac{|f(s, x, v)|^{2} e^{V(x)}}{\mathcal{M}(v)} d v d x d s \\
& -\int_{t^{\prime}}^{t}\langle K f(s), f(s)\rangle_{\mathcal{L}^{2}} d s,
\end{aligned}
$$

and the same identity holds with $f$ replaced by $f^{\delta} \in C^{0}\left(\mathbb{R}_{*}^{+} ; D(A)\right)$. We estimate separately the two different terms appearing in $\int_{t^{\prime}}^{t} \mathscr{D}\left(f^{\delta}\right) d s-\int_{t^{\prime}}^{t} \mathscr{D}(f) d s$. First, we have

$$
\begin{aligned}
& \left|\int_{t^{\prime}}^{t}\langle K f(s), f(s)\rangle_{\mathcal{L}^{2}} d s-\int_{t^{\prime}}^{t}\left\langle K f^{\delta}(s), f^{\delta}(s)\right\rangle_{\mathcal{L}^{2}} d s\right| \\
& =\left|\int_{t^{\prime}}^{t}\left\langle K\left(f(s)-f^{\delta}(s)\right), f(s)\right\rangle_{\mathcal{L}^{2}} d s+\int_{t^{\prime}}^{t}\left\langle K f^{\delta}(s),\left(f(s)-f^{\delta}(s)\right)\right\rangle_{\mathcal{L}^{2}} d s\right| \\
& \leq\left\|K\left(f-f^{\delta}\right)\right\|_{L^{2}\left(t^{\prime}, t ; \mathcal{L}^{2}\right)}\|f\|_{L^{2}\left(t^{\prime}, t ; \mathcal{L}^{2}\right)}+\left\|K f^{\delta}\right\|_{L^{2}\left(t^{\prime}, t ; \mathcal{L}^{2}\right)}\left\|f-f^{\delta}\right\|_{L^{2}\left(t^{\prime}, t ; \mathcal{L}^{2}\right)} \\
& \leq\|K\|_{\mathcal{L}^{2} \rightarrow \mathcal{L}^{2}}\left\|f-f^{\delta}\right\|_{L^{2}\left(t^{\prime}, t ; \mathcal{L}^{2}\right)}\left(\|f\|_{L^{2}\left(t^{\prime}, t ; \mathcal{L}^{2}\right)}+\left\|f^{\delta}\right\|_{L^{2}\left(t^{\prime}, t ; \mathcal{L}^{2}\right)}\right) .
\end{aligned}
$$

Using the second item above with $\mathscr{L}^{2}=\mathcal{L}^{2}$ (i.e. $\Psi=\frac{e^{V}}{\mathcal{M}}$ ) together with the fact that $\left\|f^{\delta}\right\|_{L^{2}\left(t^{\prime}, t ; \mathcal{L}^{2}\right)} \leq C\left\|f_{0}\right\|_{\mathcal{L}^{2}}$, this implies

$$
\left|\int_{t^{\prime}}^{t}\langle K f(s), f(s)\rangle_{\mathcal{L}^{2}} d s-\int_{t^{\prime}}^{t}\left\langle K f^{\delta}(s), f^{\delta}(s)\right\rangle_{\mathcal{L}^{2}} d s\right| \rightarrow 0
$$

as $\delta \rightarrow 0^{+}$. Second, taking $\mathscr{L}^{2}=L^{2}\left(\mathbb{T}^{d} \times \mathbb{R}^{d}, \Psi(x, v) d x d v\right)$ with $\Psi=$ $\left(\int_{\mathbb{R}^{d}} k\left(x, v, v^{\prime}\right) d v^{\prime}\right) \frac{e^{V(x)}}{\mathcal{M}(v)}$ in the second item above, we obtain $\left\|f^{\delta}-f\right\|_{L^{2}\left(t^{\prime}, t ; \mathscr{L}^{2}\right)} \rightarrow$ 0 and in particular 


$$
\begin{aligned}
& \int_{t^{\prime}}^{t} \int_{\mathbb{T}^{d} \times \mathbb{R}^{d}}\left(\int_{\mathbb{R}^{d}} k\left(x, v, v^{\prime}\right) d v^{\prime}\right) \frac{\left|f^{\delta}(s, x, v)\right|^{2} e^{V(x)}}{\mathcal{M}(v)} d v d x d s \\
& \rightarrow \int_{t^{\prime}}^{t} \int_{\mathbb{T}^{d} \times \mathbb{R}^{d}}\left(\int_{\mathbb{R}^{d}} k\left(x, v, v^{\prime}\right) d v^{\prime}\right) \frac{|f(s, x, v)|^{2} e^{V(x)}}{\mathcal{M}(v)} d v d x d s,
\end{aligned}
$$

as $\delta \rightarrow 0^{+}$. This finally yields $\int_{t^{\prime}}^{t} \mathscr{D}\left(f^{\delta}\right) d s \rightarrow \int_{t^{\prime}}^{t} \mathscr{D}(f) d s$, and we can take the limit $\delta \rightarrow 0^{+}$in (4.10), providing the dissipation identity (4.5) for $0<t^{\prime} \leq t$. Both terms in this identity are continuous as $t^{\prime} \rightarrow 0^{+}$so that it remains valid for $t^{\prime}=0$, which concludes the proof of the proposition.

A useful consequence of the maximum principle, of the linearity of the equation, and of Assumption $\mathbf{A} 2$ is the following statement. If $f_{0} \in \mathcal{L}^{2} \cap \mathcal{L}^{\infty}$, then the unique solution of (1.1) starting from $\left.f\right|_{t=0}=f_{0}$, satisfies

$$
\sup _{t \geq 0}\|f(t)\|_{\mathcal{L}^{\infty}} \leq\left\|f_{0}\right\|_{\mathcal{L}^{\infty}}
$$

\subsection{Weak Coercivity}

In this section, we describe some properties of the collision kernel $\mathscr{C}$ and associated dissipation $D$. In several proofs of the paper, we shall need to exploit some local coercivity properties of the dissipation. In particular, we would like to have the weak coercivity property

$$
\forall f \in \mathcal{L}^{2}, \quad \mathscr{D}(f)=0 \Longrightarrow \mathscr{C}(f)=0
$$

(and thus, $\mathscr{D}(f)=0$ is equivalent to $\mathscr{C}(f)=0$ ). A difficulty comes from the fact that in general the dissipation term does not control neither the $\mathcal{L}^{2}$ distance to the projection on the set of stationary solutions, nor the $\mathcal{L}^{2}$ norm of the collision operator.

The main result is the following lemma.

Lemma 4.5 Let $k$ be a collision kernel satisfying A1-A3. Let $T \in(0,+\infty]$ and denote $\omega=\cup_{i \in I} \omega_{i}$ the partition of $\omega$ in connected components. Then, the following three properties are equivalent

(1) $f \in C^{0}\left(0, T ; \mathcal{L}^{2}\right)$ satisfies $\mathscr{C}(f(t))=0$ for all $t \in[0, T]$.

(2) $f \in C^{0}\left(0, T ; \mathcal{L}^{2}\right)$ satisfies $\mathscr{D}(f(t))=0$ for all $t \in[0, T]$.

(3) - for all $i \in I$, we have $f(t, x, v)=\rho_{i}(t, x) \mathcal{M}(v)$ on $[0, T] \times \omega_{i}$;

- for $i, j \in I$ and $x \in \mathbb{T}^{d}$, we have: $\omega_{i}(x) \mathcal{R}_{k}^{x} \omega_{j}(x) \Longrightarrow \rho_{i}(t, x)=\rho_{j}(t, x)$ for all $t \in[0, T]$.

This lemma only states properties of the collision kernel. As such, it is not concerned with the time dependence, that we shall drop in the proof.

Proof of Lemma 4.5 By definition, we have $\mathscr{D}(f)=-2\langle\mathscr{C}(f), f\rangle_{\mathcal{L}^{2}}$ so that $(1) \Longrightarrow$ (2). 
Then, from $\mathscr{D}(f)=0$, Equation (4.2) implies that

$$
\begin{aligned}
\frac{f(x, v)}{\mathcal{M}(v)} & =\frac{f\left(x, v^{\prime}\right)}{\mathcal{M}\left(v^{\prime}\right)} \text { almost everywhere in } \\
S & :=\left\{\left(x, v, v^{\prime}\right), k\left(x, v^{\prime}, v\right)+k\left(x, v^{\prime}, v\right)>0\right\},
\end{aligned}
$$

Let $(x, v) \in \omega$. Thus, there exists $v^{\prime} \in \mathbb{R}^{d}$ such that $\left(x, v, v^{\prime}\right) \in S$. By continuity of $k$, there exists a neighborhood $U$ of $(x, v)$ such that for all $(y, w) \in U$, we have $\left(y, w, v^{\prime}\right) \in S$. Thus, for all $(y, w) \in U$, we have

$$
\frac{f(y, w)}{\mathcal{M}(w)}=\frac{f\left(y, v^{\prime}\right)}{\mathcal{M}\left(v^{\prime}\right)},
$$

that is to say that locally, $(y, w) \mapsto \frac{f(y, w)}{\mathcal{M}(w)}$ is function of $y$ only. Therefore, for all $i \in I$, there is a function $\rho_{i}$ such that $\frac{f(x, v)}{\mathcal{M}(v)}=\rho_{i}(x)$ on $\omega_{i}$.

Furthermore, take $i, j \in I$ and $x \in \mathbb{T}^{d}$ such that $\omega_{i}(x) \mathcal{R}_{k}^{x} \omega_{j}(x)$. There exists $v_{i}, v_{j} \in \mathbb{R}^{d} \times \mathbb{R}^{d}$ such that $\left(x, v_{i}\right) \in \omega_{i},\left(x, v_{j}\right) \in \omega_{j}$, and $\left(x, v_{i}, v_{j}\right) \in S$. It then follows from (4.13) and $\frac{f\left(x, v_{i}\right)}{\mathcal{M}\left(v_{i}\right)}=\rho_{i}(x), \frac{f\left(x, v_{j}\right)}{\mathcal{M}\left(v_{j}\right)}=\rho_{j}(x)$ that $\rho_{i}(x)=\rho_{j}(x)$. This concludes the proof of (2) $\Longrightarrow(3)$.

Finally, let us check that a function satisfying the two assumptions of (3) cancels the collision operator, i.e. that for all $x, v$, we have $\mathscr{C}(f)(x, v)=0$.

Let $(x, v) \in \omega$ (if $(x, v) \notin \omega$, then $\mathscr{C}(f)(x, v)=0)$. Let $i \in I$ such that $(x, v) \in \omega_{i}$. We have

$$
\begin{aligned}
\mathscr{C}(f)(x, v)= & \int \tilde{k}\left(x, v^{\prime}, v\right) f\left(x, v^{\prime}\right) d v^{\prime} \mathcal{M}(v)-\int \tilde{k}\left(x, v, v^{\prime}\right) \mathcal{M}\left(v^{\prime}\right) d v^{\prime} f(x, v) \\
= & \int \tilde{k}\left(x, v^{\prime}, v\right) f\left(x, v^{\prime}\right) d v^{\prime} \mathcal{M}(v)-\int \tilde{k}\left(x, v, v^{\prime}\right) \mathcal{M}\left(v^{\prime}\right) d v^{\prime} \rho_{i}(x) \mathcal{M}(v) \\
= & \sum_{j \in J_{i}} \int \tilde{k}\left(x, v^{\prime}, v\right) \mathbb{1}_{\omega_{j}}\left(x, v^{\prime}\right) f\left(x, v^{\prime}\right) d v^{\prime} \mathcal{M}(v) \\
& -\int \tilde{k}\left(x, v, v^{\prime}\right) \mathcal{M}\left(v^{\prime}\right) d v^{\prime} \rho_{i}(x) \mathcal{M}(v) \\
= & \sum_{j \in J_{i}} \int \tilde{k}\left(x, v^{\prime}, v\right) \mathbb{1}_{\omega_{j}}\left(x, v^{\prime}\right) \mathcal{M}\left(v^{\prime}\right) d v^{\prime} \rho_{j}(x) \mathcal{M}(v) \\
& -\int \tilde{k}\left(x, v, v^{\prime}\right) \mathcal{M}\left(v^{\prime}\right) d v^{\prime} \rho_{i}(x) \mathcal{M}(v),
\end{aligned}
$$

where $J_{i}$ is the largest subset of $I$ such that for all $j \in J_{i}$, there exists $v^{\prime} \in \mathbb{R}^{d}$ such that $\left(x, v^{\prime}\right) \in \omega_{j}$ and $\tilde{k}\left(x, v^{\prime}, v\right)>0$. According to the second property satisfied by $f$, for all $j \in J_{i}$,

$$
\rho_{j}(x)=\rho_{i}(x)
$$


and thus we deduce

$$
\begin{aligned}
\mathscr{C}(f)(x, v)= & \sum_{j \in J_{i}} \int \tilde{k}\left(x, v^{\prime}, v\right) \mathbb{1}_{\omega_{j}}\left(x, v^{\prime}\right) \mathcal{M}\left(v^{\prime}\right) d v^{\prime} \rho_{i}(x) \mathcal{M}(v) \\
& -\int \tilde{k}\left(x, v, v^{\prime}\right) \mathcal{M}\left(v^{\prime}\right) d v^{\prime} \rho_{i}(x) \mathcal{M}(v) \\
= & \left(\int \tilde{k}\left(x, v^{\prime}, v\right) \mathcal{M}\left(v^{\prime}\right) d v^{\prime}-\int \tilde{k}\left(x, v, v^{\prime}\right) \mathcal{M}\left(v^{\prime}\right) d v^{\prime}\right) \rho_{i}(x) \mathcal{M}(v) \\
= & 0 .
\end{aligned}
$$

The last line comes from the fact that $k$ satisfies A2. This concludes the proof of (3) $\Longrightarrow(1)$.

Remark 4.1 Another benefit of Lemma 4.5 is that it allows to rephrase the Unique Continuation Property, in a slightly more explicit way.

Denote $\omega=\cup_{i \in I} \omega_{i}$ the partition of $\omega$ in connected components. The set $\omega$ satisfies the Unique Continuation Property if and only if the following holds. The only solution $f \in C^{0}\left(\mathbb{R} ; \mathcal{L}^{2}\right)$ to

$$
\partial_{t} f+v \cdot \nabla_{x} f-\nabla_{x} V \cdot \nabla_{v} f=0
$$

satisfying the following properties

- for all $i \in I, f(t, x, v)=\rho_{i}(t, x) \mathcal{M}(v)$ on $[0, T] \times \omega_{i}$;

- for $i, j \in I$ and $x \in \mathbb{T}^{d}, \omega_{i}(x) \mathcal{R}_{k}^{x} \omega_{j}(x) \Longrightarrow \rho_{i}(t, x)=\rho_{j}(t, x)$ for all $t \in[0, T]$, is $f=\left(\int_{\mathbb{T}^{d} \times \mathbb{R}^{d}} f d v d x\right) e^{-V(x)} \mathcal{M}(v)$.

Remark 4.2 If $\tilde{k}$ is in $L^{\infty}$ (but only in this case), we can actually prove a stronger result, namely that the dissipation controls the norm of a "symmetrized" collision operator. To state and prove such a result, we introduce the symmetrized collision kernel

$$
\bar{k}\left(x, v^{\prime}, v\right):=\frac{\tilde{k}\left(x, v^{\prime}, v\right)+\tilde{k}\left(x, v, v^{\prime}\right)}{2}, \quad k^{*}\left(x, v^{\prime}, v\right):=\bar{k}\left(x, v^{\prime}, v\right) \mathcal{M}(v)
$$

Note in particular that $\bar{k}\left(x, v^{\prime}, v\right) \in L^{\infty}\left(\mathbb{T}^{d} \times \mathbb{R}^{d} \times \mathbb{R}^{d}\right)$ if $\tilde{k} \in L^{\infty}\left(\mathbb{T}^{d} \times \mathbb{R}^{d} \times \mathbb{R}^{d}\right)$.

We also introduce the associated collision operator

$$
\left.\mathcal{C}(f):=\int_{\mathbb{R}^{d}}\left[k^{*}\left(x, v^{\prime}, v\right) f\left(v^{\prime}\right)-k^{*}\left(x, v, v^{\prime}\right)\right) f(v)\right] d v^{\prime} .
$$

Note that we have $\mathcal{C}(f)=\mathscr{C}(f)$ if and only if $\bar{k}\left(x, v^{\prime}, v\right)=\tilde{k}\left(x, v^{\prime}, v\right)$, i.e. if $\tilde{k}\left(x, v^{\prime}, v\right)$ is symmetric with respect to $v$ and $v^{\prime}$ (this corresponds to the class E1). 
Lemma 4.6 Let $k$ be a collision kernel satisfying A1-A2, and such that $\tilde{k} \in L^{\infty}$. For any $f \in \mathcal{L}^{2}$, we have

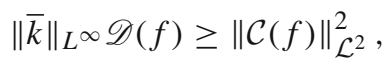

Proof of Lemma 4.6 According to the definition of the dissipation (4.2) and the symmetry of $\bar{k}$, we have

$$
\mathscr{D}(f) \geq \int_{\mathbb{T}^{d}} e^{V} \int_{\mathbb{R}^{d}} \int_{\mathbb{R}^{d}} \bar{k}\left(x, v^{\prime}, v\right) \mathcal{M} \mathcal{M}^{\prime}\left(\frac{f}{\mathcal{M}}-\frac{f^{\prime}}{\mathcal{M}^{\prime}}\right)^{2} d v^{\prime} d v d x
$$

and hence

$$
\|\bar{k}\|_{L^{\infty}} \mathscr{D}(f) \geq \int_{\mathbb{T}^{d}} e^{V} \int_{\mathbb{R}^{d}} \int_{\mathbb{R}^{d}} \bar{k}^{2}\left(x, v^{\prime}, v\right) \mathcal{M} \mathcal{M}^{\prime}\left(\frac{f}{\mathcal{M}}-\frac{f^{\prime}}{\mathcal{M}^{\prime}}\right)^{2} d v^{\prime} d v d x
$$

By Jensen's inequality it follows that

$$
\begin{aligned}
\|\bar{k}\|_{L^{\infty}} \mathscr{D}(f) & \geq \int_{\mathbb{T}^{d}} e^{V} \int_{\mathbb{R}^{d}} \mathcal{M}(v)\left(\int_{\mathbb{R}^{d}} \bar{k}\left(x, v^{\prime}, v\right) \mathcal{M}\left(v^{\prime}\right)\left(\frac{f(v)}{\mathcal{M}(v)}-\frac{f\left(v^{\prime}\right)}{\mathcal{M}\left(v^{\prime}\right)}\right) d v^{\prime}\right)^{2} d v d x \\
& =\int_{\mathbb{T}^{d}} e^{V} \int_{\mathbb{R}^{d}} \frac{1}{\mathcal{M}(v)} \mathcal{C}(f)^{2} d v d x \\
& =\|\mathcal{C}(f)\|_{\mathcal{L}^{2}}^{2},
\end{aligned}
$$

where we used again the symmetry of $\bar{k}$. This concludes the proof of the lemma.

\section{Characterization of Convergence to Equilibrium}

In this Section, we shall first give a proof of Theorem 2.2; then we will provide a proof of Theorem 2.1, which will be a consequence of our main result in this direction, namely Theorem 5.1 .

We start with a technical lemma concerning the evolution under the flow of the connected components of $\bigcup_{s \in \mathbb{R}^{+}} \phi_{-s}(\omega)$.

Lemma 5.1 Set $\tilde{\Omega}=\bigcup_{s \in \mathbb{R}^{+}} \phi_{-s}(\omega)$ and denote by $\left(\Omega_{i}\right)_{i \in I}$ the partition of $\tilde{\Omega}$ in connected components, and $\mathcal{A}=\mathbb{T}^{d} \times \mathbb{R}^{d} \backslash \tilde{\Omega}$. Then we have for all $t \geq 0$, for all $i \in I$,

$$
\phi_{-t}(\tilde{\Omega}) \subset \tilde{\Omega}, \quad \phi_{t}(\mathcal{A}) \subset \mathcal{A}, \quad \phi_{-t}\left(\Omega_{i}\right) \subset \Omega_{i} .
$$

If moreover $\omega$ satisfies a.e.i.t. GCC (i.e. $\mathcal{A}$ has zero Lebesgue measure), then for all $i \in I$, for all $t \in \mathbb{R}$,

$$
\phi_{t}\left(\Omega_{i}\right)=\Omega_{i} \text { up to a set of measure zero. }
$$


Proof of Lemma 5.1 First, we just remark that for all $t \geq 0$, we have $\phi_{-t}(\tilde{\Omega})=$ $\bigcup_{s \in \mathbb{R}^{+}} \phi_{-t-s}(\omega) \subset \tilde{\Omega}$. Taking the complement of this inclusion yields for $t \geq 0$, $\phi_{t}(\mathcal{A}) \subset \mathcal{A}$.

Let us now fix $i \in I$ and prove that

$$
\text { for all } t \geq 0, \quad \phi_{-t}\left(\Omega_{i}\right) \subset \Omega_{i} \text {. }
$$

Take $(x, v) \in \Omega_{i}$ and $t>0$. If $\phi_{-t}(x, v) \notin \Omega_{i}$, then there exists $t_{0} \in(0, t]$ such that $\phi_{-t_{0}}(x, v) \notin \tilde{\Omega}$ since $\Omega_{i}$ is a connected component of this set. This is in contradiction with $\phi_{-t_{0}}(\tilde{\Omega}) \subset \tilde{\Omega}$. This implies (5.3).

Finally, if $\mathcal{A}$ has zero Lebesgue measure, then $\left|\phi_{-t}(\mathcal{A})\right|=0$ as well. As a consequence, the identity

$$
\mathbb{T}^{d} \times \mathbb{R}^{d}=\phi_{-t}\left(\mathbb{T}^{d} \times \mathbb{R}^{d}\right)=\phi_{-t}\left(\bigcup_{i \in I} \Omega_{i} \cup \mathcal{A}\right)=\bigcup_{i \in I} \phi_{-t}\left(\Omega_{i}\right) \cup \phi_{-t}(\mathcal{A})
$$

yields $\mathbb{T}^{d} \times \mathbb{R}^{d}=\bigcup_{i \in I} \phi_{-t}\left(\Omega_{i}\right)$ up to a set of measure zero. Since for all $i \in I$ and $t \geq 0, \phi_{-t}\left(\Omega_{i}\right) \subset \Omega_{i}$, we obtain (still for $t \geq 0$ ) $\phi_{-t}\left(\Omega_{i}\right)=\Omega_{i}$ up to a set of measure zero, from which the conclusion of the lemma follows.

Remark 5.1 Note that if $V=0$ and $\omega$ satisfies the following property: $(x, v) \in \omega \Leftrightarrow$ $(x,-v) \in \omega$, then the inclusions in (5.1) become equalities. Hence, all sets considered in (5.1) are then invariant by $\phi_{t}$ for all $t \in \mathbb{R}$.

\subsection{Proof of Theorem 2.2}

We shall prove that $($ i. $) \Longrightarrow($ iii.), that (iii.) $\Longrightarrow$ (ii.) and finally that $($ ii. $) \Longrightarrow$ (i.).

(i.) $\Longrightarrow$ (iii.) We prove that the Unique Continuation Property (of Definition 2.11) implies the decay.

We first prove the expected convergence for data enjoying more regularity, i.e. we prove

$$
\text { for all } f_{0} \in \mathcal{L}^{2} \cap \mathcal{L}^{\infty}, \quad\left\|f(t)-\left(\int_{\mathbb{T}^{d} \times \mathbb{R}^{d}} f_{0} d v d x\right) e^{-V} \mathcal{M}\right\|_{\mathcal{L}^{2}} \rightarrow_{t \rightarrow+\infty} 0 .
$$

Since (1.1) is linear,

$$
g(t):=f(t)-\left(\int_{\mathbb{T}^{d} \times \mathbb{R}^{d}} f_{0} d v d x\right) e^{-V} \mathcal{M}
$$

is a solution to (1.1) with initial datum

$$
g(0)=f(0)-\left(\int_{\mathbb{T}^{d} \times \mathbb{R}^{d}} f_{0} d v d x\right) e^{-V} \mathcal{M} \in \mathcal{L}^{2} \cap \mathcal{L}^{\infty}
$$


satisfying $\int g(0) d v d x=0$. Therefore proving (5.4) is equivalent to proving that

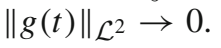

We argue by contradiction. Assume that there exists an initial datum $g_{0}$ in $\mathcal{L}^{2} \cap \mathcal{L}^{\infty}$ (with $\int g_{0} d v d x=0$ ), $\varepsilon>0$ and an increasing sequence $\left(t_{n}\right)_{n \in \mathbb{N}}$ such that:

$$
t_{n} \geq e^{n}, \quad \text { and } \quad\left\|g_{0}\right\|_{\mathcal{L}^{2}} \geq\left\|g\left(t_{n}\right)\right\|_{\mathcal{L}^{2}}>\varepsilon .
$$

From this sequence, we may extract a subsequence (still denoted $\left(t_{n}\right)$ ) satisfying

$$
t_{n+1}-t_{n} \rightarrow+\infty
$$

According to the Maximum Principle of Proposition 4.1, we have

$$
\text { for all } t \geq 0, \quad\|g(t)\|_{\mathcal{L}^{\infty}} \leq\left\|g_{0}\right\|_{\mathcal{L}^{\infty}}
$$

We introduce the shifted function

$$
h_{n}(t, x, v):=g\left(t_{n}+t, x, v\right) .
$$

By the time translation invariance of (1.1), $h_{n}$ is still a solution to (1.1), with initial datum $h_{n}(0)=g\left(t_{n}\right)$. Using (5.5), up to some extraction, we can assume that there is $\alpha \in\left[\varepsilon,\left\|g_{0}\right\|_{\mathcal{L}^{2}}\right]$ such that

$$
\left\|h_{n}(0)\right\|_{\mathcal{L}^{2}} \rightarrow_{n \rightarrow+\infty} \alpha
$$

Note also that by conservation of the mass, for all $n \in \mathbb{N}$ and all $t \geq 0$,

$$
\int h_{n}(t) d v d x=\int g_{0} d v d x=0
$$

Using the dissipation identity (4.5) for $g$, we have:

$$
\left\|g\left(t_{n+1}\right)\right\|_{\mathcal{L}^{2}}^{2}-\left\|g\left(t_{n}\right)\right\|_{\mathcal{L}^{2}}^{2}=-\int_{t_{n}}^{t_{n+1}} \mathscr{D}(g) d t
$$

that is (using the time translation invariance):

$$
\left\|h_{n+1}(0)\right\|_{\mathcal{L}^{2}}^{2}-\left\|h_{n}(0)\right\|_{\mathcal{L}^{2}}^{2}=-\int_{0}^{t_{n+1}-t_{n}} \mathscr{D}\left(h_{n}\right) d t .
$$

This, together with (5.6) and (5.8), implies that for any $T>0$,

$$
\int_{0}^{T} \mathscr{D}\left(h_{n}\right) d t \rightarrow 0
$$


Now, up to another extraction, since for any $n \in \mathbb{N}$,

$$
\text { for all } t \geq 0, \quad\left\|h_{n}(t)\right\|_{\mathcal{L}^{2}} \leq\left\|g_{0}\right\|_{\mathcal{L}^{2}},
$$

we can assume that $h_{n} \rightarrow h$ weakly in $L_{t, l o c}^{2} \mathcal{L}^{2}$. Let us now prove that $h=0$. First, since $h_{n}$ is a solution to (1.1), by linearity, $h$ also satisfies (1.1). Then, according to Lemma 4.2, we have

$$
\|\mathscr{D}(h)\|_{L^{1}(0, T)} \leq \liminf _{n \rightarrow+\infty}\left\|\mathscr{D}\left(h_{n}\right)\right\|_{L^{1}(0, T)}=0 .
$$

Thus, by weak coercivity (see Lemma 4.5), we infer that $\mathscr{C}(h)=0$ on $[0, T]$, for any $T>0$, and therefore $h$ satisfies the kinetic transport equation (2.6). Using the Unique Continuation Property (see Definition 2.11), we deduce that

$$
h=\left(\int h d v d x\right) \frac{e^{V}}{\mathcal{M}} .
$$

Since $h_{n} \rightarrow h$ weakly in $L_{t}^{2} \mathcal{L}^{2}$, using (5.9), we obtain in particular that for any $T>0$

$$
\int_{0}^{T}\left(\int h d v d x\right) d t=0 .
$$

Since $\int_{0}^{T}\left(\int h d v d x\right) d t=T\left(\int h(0) d v d x\right)$, we deduce that $\int h d v d x=0$ so that $h=0$.

Let us now consider the sequence of defect measures $v_{n}:=\left|h_{n}\right|^{2}$, which, according to (5.11) and (5.7) satisfies, for all $n \in \mathbb{N}$,

$$
\text { for all } t \geq 0, \quad\left\|v_{n}(t)\right\|_{\mathcal{L}^{1}} \leq\|g(0)\|_{\mathcal{L}^{2}}^{2}, \quad\left\|v_{n}(t)\right\|_{\mathcal{L}^{\infty}} \leq C_{0}\|g(0)\|_{\mathcal{L}^{\infty}}^{2}
$$

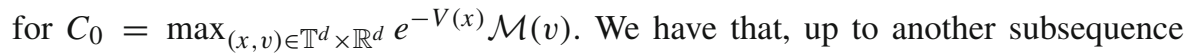
$v_{n} \rightarrow v$ weakly- $\star$ in $L_{t, l o c}^{\infty} \mathcal{L}^{\infty}$. Let us compute the equation satisfied by $v$ : to this purpose, we consider (1.1) satisfied by $h_{n}$ and multiply it by $h_{n}$. We obtain:

$$
\begin{aligned}
\partial_{t} v_{n} & +v \cdot \nabla_{x} v_{n}-\nabla_{x} V \cdot \nabla_{v} v_{n} \\
& =2\left[\int_{\mathbb{R}^{d}}\left[k\left(x, v^{\prime}, v\right) h_{n}\left(v^{\prime}\right)-k\left(x, v, v^{\prime}\right) h_{n}(v)\right] d v^{\prime}\right] h_{n} \\
& =2\left(\int_{\mathbb{R}^{d}} k\left(x, v^{\prime}, v\right) h_{n}\left(v^{\prime}\right) d v^{\prime}\right) h_{n}-2\left(\int_{\mathbb{R}^{d}} k\left(x, v, v^{\prime}\right) d v^{\prime}\right) v_{n} .
\end{aligned}
$$

Using the averaging lemma of Corollary 12.2 and the fact that $h_{n}$ weakly converges to 0 , we deduce that

$$
\int_{\mathbb{R}^{d}} k\left(x, v^{\prime}, v\right) h_{n}\left(t, x, v^{\prime}\right) d v^{\prime} \rightarrow 0
$$


strongly in $L_{t, l o c}^{2} \mathcal{L}^{2}$. On the other hand, according to $(5.11)$, the sequence $\left(h_{n}\right)$ is uniformly bounded in $L_{t, l o c}^{2} \mathcal{L}^{2}$. We hence obtain

$$
\left(\int_{\mathbb{R}^{d}} k\left(x, v^{\prime}, v\right) h_{n}\left(t, x, v^{\prime}\right) d v^{\prime}\right) h_{n} \rightarrow 0 \text { strongly in } L_{t, l o c}^{1} \mathcal{L}^{1}
$$

The second term, by definition of $v$, weakly converges to $-2\left(\int_{\mathbb{R}^{d}} k\left(x, v, v^{\prime}\right) d v^{\prime}\right) v$ in the sense of distributions, so that $v$ satisfies the equation

$$
\partial_{t} v+v \cdot \nabla_{x} v-\nabla_{x} V \cdot \nabla_{v} v=-2\left(\int_{\mathbb{R}^{d}} k\left(x, v, v^{\prime}\right) d v^{\prime}\right) v .
$$

Moreover, writing

$$
\left(\int_{\mathbb{R}^{d}} k\left(x, v, v^{\prime}\right) d v^{\prime}\right)\left|h_{n}\right|^{2}=-\mathscr{C}\left(h_{n}\right) h_{n}+\left(\int_{\mathbb{R}^{d}} k\left(x, v^{\prime}, v\right) h_{n}\left(t, x, v^{\prime}\right) d v^{\prime}\right) h_{n},
$$

and using (5.10) together with (5.14), we deduce that

$$
\left(\int_{\mathbb{R}^{d}} k\left(x, v, v^{\prime}\right) d v^{\prime}\right) v=0 .
$$

Thus, (5.15) combined with (5.17) entails that $v$ satisfies the kinetic transport equation

$$
\partial_{t} v+v \cdot \nabla_{x} v-\nabla_{x} V \cdot \nabla_{v} v=0
$$

which also shows that $v \in C_{t}^{0}\left(\mathcal{L}^{2}\right)$. This, combined with the fact that $v=0$ on $\mathbb{R}^{+} \times \omega$ (again coming from (5.17)) and the Unique Continuation Property, implies

$$
v=\left(\int v(0) d v d x\right) e^{-V(x)} \mathcal{M}(v)
$$

According to (5.17), this means that $v=0$.

We now prove that there is no loss of mass at infinity. Let $R>0$. We have:

$$
\begin{aligned}
\int_{\mathbb{T}^{d} \times \mathbb{R}^{d}} v_{n}(0) \frac{e^{V}}{\mathcal{M}} \mathbb{1}_{v \in \mathbb{R}^{d} \backslash B(0, R)} d v d x & \leq\|g(0)\|_{\mathcal{L}^{\infty}}^{2} \int_{\mathbb{T}^{d} \times \mathbb{R}^{d}} \frac{\mathcal{M}}{e^{V}} \mathbb{1}_{v \in \mathbb{R}^{d} \backslash B(0, R)} d v d x \\
& \leq\|g(0)\|_{\mathcal{L}^{\infty}}^{2} \int_{\mathbb{T}^{d}} e^{-V} d x \int_{|v| \geq R} \mathcal{M}(v) d v
\end{aligned}
$$

which is exponentially converging to zero as $R \rightarrow \infty$. This yields

$$
\lim _{R \rightarrow \infty} \int_{\mathbb{T}^{d} \times \mathbb{R}^{d}} v_{n}(0) \frac{e^{V}}{\mathcal{M}} \mathbb{1}_{v \in \mathbb{R}^{d} \backslash B(0, R)} d v d x=0 .
$$


Therefore, on the one hand, up to a subsequence, we can assume that $v_{n}(0) \frac{e^{V}}{\mathcal{M}} \rightarrow$ $v(0) \frac{e^{V}}{\mathcal{M}}$ tightly in $\mathcal{M}_{x, v}^{+}$and thus

$$
\int_{\mathbb{T}^{d} \times \mathbb{R}^{d}} v_{n}(0) \frac{e^{V}}{\mathcal{M}} d v d x \rightarrow \int_{\mathbb{T}^{d} \times \mathbb{R}^{d}} v(0) \frac{e^{V}}{\mathcal{M}} d v d x=0 .
$$

On the other hand, using (5.8), we have

$$
\int_{\mathbb{T}^{d} \times \mathbb{R}^{d}} v_{n}(0) \frac{e^{V}}{\mathcal{M}} d v d x \rightarrow \alpha>0
$$

This yields a contradiction, and concludes the proof of (5.4).

We finally deduce (2.8) by an approximation argument. Let $f_{0} \in \mathcal{L}^{2}$ and $f(t)$ be the solution associated to $f_{0}$. Let $\varepsilon>0$. There exists $f_{0, \varepsilon} \in \mathcal{L}^{2} \cap \mathcal{L}^{\infty}$ such that $\left\|f_{0}-f_{0, \varepsilon}\right\|_{\mathcal{L}^{2}} \leq \varepsilon$. Let $f_{\varepsilon}(t)$ be the solution associated to $f_{0, \varepsilon}$. Since $f-f_{\varepsilon}$ is a solution of (1.1) with initial datum $f_{0}-f_{0, \varepsilon}$ we also have for any $t \geq 0,\left\|f(t)-f_{\varepsilon}(t)\right\|_{\mathcal{L}^{2}} \leq \varepsilon$.

By (5.4), there exists $t_{0} \geq 0$ such that for all $t \geq t_{0}$,

$$
\left\|f_{\varepsilon}(t)-\int_{\mathbb{T}^{d} \times \mathbb{R}^{d}} f_{0, \varepsilon} d v d x e^{-V(x)} \mathcal{M}(v)\right\|_{\mathcal{L}^{2}} \leq \varepsilon
$$

Thus, it follows that for all $t \geq t_{0}$,

$$
\begin{aligned}
& \left\|f(t)-\left(\int_{\mathbb{T}^{d} \times \mathbb{R}^{d}} f_{0} d v d x\right) e^{-V(x)} \mathcal{M}(v)\right\|_{\mathcal{L}^{2}} \\
& \leq\left\|f(t)-f_{\varepsilon}(t)\right\|_{\mathcal{L}^{2}}+\left\|f_{\varepsilon}(t)-\int_{\mathbb{T}^{d} \times \mathbb{R}^{d}} f_{0, \varepsilon} d v d x e^{-V(x)} \mathcal{M}(v)\right\|_{\mathcal{L}^{2}} \\
& \quad+\left\|\int_{\mathbb{T}^{d} \times \mathbb{R}^{d}}\left(f_{0, \varepsilon}-f_{0}\right) d v d x e^{-V(x)} \mathcal{M}(v)\right\|_{\mathcal{L}^{2}} \\
& \leq 2 \varepsilon+\int_{\mathbb{T}^{d} \times \mathbb{R}^{d}}\left|f_{0}-f_{0, \varepsilon}\right| d v d x .
\end{aligned}
$$

Besides, we have

$$
\left(\int_{\mathbb{T}^{d} \times \mathbb{R}^{d}}\left|f_{0}-f_{0, \varepsilon}\right| d v d x\right)^{2} \leq\left\|f_{0}-f_{0, \varepsilon}\right\|_{\mathcal{L}^{2}}^{2}\left(\int_{\mathbb{T}^{d} \times \mathbb{R}^{d}} e^{-V} \mathcal{M}(v) d x d v\right)
$$

The last two inequalities together yield, for all $t \geq t_{0}$,

$$
\left\|f(t)-\left(\int_{\mathbb{T}^{d} \times \mathbb{R}^{d}} f_{0} d v d x\right) e^{-V(x)} \mathcal{M}(v)\right\|_{\mathcal{L}^{2}} \leq 3 \varepsilon,
$$

which concludes the proof of (2.8). 
(iii) $\Rightarrow$ (ii.) Assume that (ii.) does not hold. Either $\mathbb{T}^{d} \times \mathbb{R}^{d} \backslash \bigcup_{s \in \mathbb{R}^{+}} \phi_{-s}(\omega)$ has positive Lebesgue measure, or the equivalence relation $\approx$ has two or more equivalence classes (or equivalently, by Lemma 3.1, the binary relation $\sim$ has two or more equivalence classes).

Suppose first that $\mathcal{A}:=\mathbb{T}^{d} \times \mathbb{R}^{d} \backslash \bigcup_{s \in \mathbb{R}^{+}} \phi_{-s}(\omega)$ has positive Lebesgue measure. We set

$$
f_{0}(x, v)=\mathbb{1}_{\mathcal{A}}(x, v) e^{-V(x)} \mathcal{M}(v) .
$$

Note that $f_{0}$ satisfies $\int_{\mathbb{T}^{d} \times \mathbb{R}^{d}} f_{0}(x, v) d x d v>0$ as $\mathcal{A}$ is of positive Lebesgue measure. We consider $f(t, \cdot)$ the solution to $(5.20)$ with initial datum $f_{0}$, given by

$$
f(t, x, v)=f_{0} \circ \phi_{-t}(x, v)=\mathbb{1}_{\phi_{t}(\mathcal{A})}(x, v) e^{-V(x)} \mathcal{M}(v)
$$

Moreover, for all $t \geq 0$, we have $\phi_{t}(\mathcal{A}) \cap \omega=\emptyset$ since $\mathcal{A} \cap \phi_{-t}(\omega)=\emptyset$. Therefore, $f=0$ on $\mathbb{R}^{+} \times \omega$ so that, according to the characterization of $\omega$ in $(2.2), \mathscr{C}(f)=0$ and $f$ is also a solution of (1.1). Moreover, this implies that

$$
\left\|f(t)-\left(\int_{\mathbb{T}^{d} \times \mathbb{R}^{d}} f_{0} d v d x\right) e^{-V(x)} \mathcal{M}(v)\right\|_{\mathcal{L}^{2}} \not \rightarrow_{t \rightarrow+\infty} 0
$$

Thus, (iii.) does not hold.

Suppose now that $\mathbb{T}^{d} \times \mathbb{R}^{d} \backslash \bigcup_{s \in \mathbb{R}^{+}} \phi_{-s}(\omega)$ has zero Lebesgue measure and that the equivalence relation $\sim$ has (at least) two distinct equivalence classes, say $\left[\Omega_{1}\right]$ and $\left[\Omega_{2}\right]$.

We define now a function $f(x, v)$ as follows

$$
f(x, v)=\sum_{\Omega^{\prime} \in\left[\Omega_{1}\right]} \mathbb{1}_{\Omega^{\prime}}(x, v) e^{-V(x)} \mathcal{M}(v) .
$$

Using (5.2) in Lemma 5.1, we deduce that for all $t \geq 0$,

$$
\begin{aligned}
f \circ \phi_{t}(x, v) & =\sum_{\Omega^{\prime} \in\left[\Omega_{1}\right]} \mathbb{1}_{\Omega^{\prime}}\left(\phi_{t}(x, v)\right) e^{-V(x)} \mathcal{M}(v)=\sum_{\Omega^{\prime} \in\left[\Omega_{1}\right]} \mathbb{1}_{\Omega^{\prime}}(x, v) e^{-V(x)} \mathcal{M}(v) \\
& =f(x, v),
\end{aligned}
$$

so that $f$ is a stationary solution of the Vlasov equation $\partial_{t} f+v \cdot \nabla_{x} f-\nabla_{x} V \cdot \nabla_{v} f=0$.

There remains to prove that $f$ cancels the collision operator. Denote $\omega=\cup_{i \in I} \omega_{i}$ the partition of $\omega$ in connected components.

By Lemma 4.5, $f$ cancels the collision operator if and only if $f$ satisfies the following two properties.

(1) For all $i \in I$,

$$
f=\rho_{i}(x) \mathcal{M}(v) \text { on } \omega_{i},
$$


(2) For $i, j \in I$, and $x \in \mathbb{T}^{d}, \omega_{i}(x) \mathcal{R}_{k}^{x} \omega_{j}(x) \Longrightarrow \rho_{i}(x)=\rho_{j}(x)$.

We check now that the function $f$ defined in (5.19) satisfies these two properties.

Let $i \in I$. If for all $\Omega^{\prime} \in\left[\Omega_{1}\right], \omega_{i} \cap \Omega^{\prime}=\emptyset$, then $f=0$ on $\omega_{i}$ (and hence satisfies (1) with $\rho_{i}=0$ ). If there is $\Omega^{\prime} \in\left[\Omega_{1}\right]$ such that $\omega_{i} \cap \Omega^{\prime} \neq \emptyset$, then since $\Omega^{\prime}$ is a connected component of $\bigcup_{s \in \mathbb{R}^{+}} \phi_{-s}(\omega)$, we have $\omega_{i} \subset \Omega^{\prime}$. Thus we have $f=e^{-V} \mathcal{M}$ on $\omega_{i}$ (and hence $f$ satisfies (1) with $\rho_{i}=e^{-V}$ ). We deduce that for all $i \in I, f$ is of the form $\rho_{i}(x) \mathcal{M}(v)$ on $\omega_{i}$.

Take now $i, j \in I$ and $x \in \mathbb{T}^{d}$ such that $\omega_{i}(x) \mathcal{R}_{k}^{x} \omega_{j}(x)$. Let $\Omega^{(i)}$ (resp. $\Omega^{(j)}$ ) be the connected component of $\bigcup_{s \in \mathbb{R}^{+}} \phi_{-s}(\omega)$ which contains $\omega_{i}$ (resp. $\omega_{j}$ ). By definition of the relations $\mathcal{R}_{k}$ and $\mathcal{R}_{k}^{x}$, this directly yields $\Omega^{(i)} \mathcal{R}_{k} \Omega^{(j)}$, and a fortiori we deduce that $\Omega^{(i)} \sim \Omega^{(j)}$ : in other words, these are in the same equivalence class for $\sim$. According to the definition of $f$, this implies $\rho_{i}(x)=\rho_{j}(x)$ (which is equal to $e^{-V(x)}$ if $\Omega^{(i, j)} \in\left[\Omega_{1}\right]$ and to 0 if not).

Therefore, the function $f$ satisfies the two properties and, by Lemma 4.5, cancels the collision operator.

However, we have

$$
\cup_{\Omega^{\prime} \in\left[\Omega_{2}\right]} \Omega^{\prime} \subset\left(\mathbb{T}^{d} \times \mathbb{R}^{d} \backslash \cup_{\Omega^{\prime} \in\left[\Omega_{1}\right]} \Omega^{\prime}\right),
$$

and $\cup_{\Omega^{\prime} \in\left[\Omega_{2}\right]} \Omega^{\prime}$ has a positive Lebesgue measure. Consequently, the measure of $\mathbb{T}^{d} \times$ $\mathbb{R}^{d} \backslash \cup_{\Omega^{\prime} \in\left[\Omega_{1}\right]} \Omega^{\prime}$ is positive, so that $f$ is a stationary solution of (1.1) which is not a uniform Maxwellian. As a consequence, (iii.) does not hold.

(ii.) $\Rightarrow\left(\right.$ i.) Assume that (ii.) holds. Let $f \in C_{t}^{0}\left(\mathcal{L}^{2}\right)$ be a solution to

$$
\begin{aligned}
& \partial_{t} f+v \cdot \nabla_{x} f-\nabla_{x} V \cdot \nabla_{v} f=0, \\
& \mathscr{C}(f)=0 .
\end{aligned}
$$

As usual, without loss of generality, we can assume that $\int_{\mathbb{T}^{d} \times \mathbb{R}^{d}} f d v d x=0$. The goal is to show that $f=0$.

Since $f$ cancels the collision operator, by Lemma 4.5, the restriction of $f$ to $\omega$ is necessarily of the form

$$
f_{\mid \omega}(t, x, v)=\sum_{i \in I} \mathbb{1}_{\omega_{i}}(x, v) \rho_{i}(t, x) \mathcal{M}(v)
$$

where $\omega=\bigcup_{i \in I} \omega_{i}$ is the partition of $\omega$ in connected components. Furthermore, for $i, j \in I$, if there is $x \in \mathbb{T}^{d}$ such that $\omega_{i}(x) \mathcal{R}_{k}^{x} \omega_{j}(x)$, then $\rho_{i}(t, x)=\rho_{j}(t, x)$.

Consider now $\tilde{\omega}$ a connected component of $\omega$. For $(t, x, v) \in \mathbb{R}^{+} \times \tilde{\omega}$, remark that the function $g(t, x):=\frac{e^{V}}{\mathcal{M}(v)} f$ does not depend on the variable $v$ according to (5.22). We have, in the sense of distributions in $\mathbb{R}^{+} \times \tilde{\omega}$,

$$
\partial_{t} g+v \cdot \nabla_{x} g=\frac{e^{V}}{\mathcal{M}(v)}\left[\partial_{t} f+v \cdot \nabla_{x} f+\left(v \cdot \nabla_{x} V\right) f\right]
$$


Since $f$ satisfies (5.20), this implies that $g$ satisfies the free transport equation

$$
\partial_{t} g+v \cdot \nabla_{x} g=0
$$

in the sense of distributions in $\mathbb{R}^{+} \times \tilde{\omega}$.

Let $(x, v) \in \tilde{\omega}$. Since $\tilde{\omega}$ is open, there exists $\delta>0$ such that $B(x, \delta) \times B(v, \delta) \subset \tilde{\omega}$ and $\eta>0$ such that for all $t \in(-\eta, \eta)$, for all $\left(x^{\prime}, v^{\prime}\right) \in B(x, \delta) \times B(v, \delta)$, we have $\left(x^{\prime}+t v^{\prime}, v^{\prime}\right) \in \tilde{\omega}$. Integrating (5.23) along characteristics we obtain

$$
g\left(t, x^{\prime}\right)=g\left(0, x^{\prime}+t v^{\prime}\right), \quad \text { for }\left(t, x^{\prime}, v^{\prime}\right) \in(-\eta, \eta) \times B(x, \delta) \times B(v, \delta) .
$$

Setting $U_{x}:=\left\{x-\frac{\eta}{2} v+\frac{\eta}{2} v^{\prime}, v^{\prime} \in B(v, \delta)\right\}$, we remark that $U_{x}$ is an open set containing $x$. Moreover, for all $y \in U_{x}$, we have $y=x-\frac{\eta}{2} v+\frac{\eta}{2} v^{\prime}$ for some $v^{\prime} \in B(v, \delta)$ so that, using (5.24), we have

$$
g(0, y)=g\left(0, x-\frac{\eta}{2} v+\frac{\eta}{2} v^{\prime}\right)=g\left(\eta / 2, x-\frac{\eta}{2} v\right) .
$$

Hence, $g(0, \cdot)$ is constant on $U_{x}$, and therefore constant on $\tilde{\omega}$ (since $\tilde{\omega}$ is connected).

Using the time translation invariance of (5.23), we also have that for all $t \geq 0$, $g(t, \cdot)$ is locally constant on $\omega$. As a consequence, for all $t \geq 0, \frac{e^{V}}{\mathcal{M}} f(t, \cdot)$ is locally constant on $\omega$, which means that $\rho_{i}(t, x)=\kappa_{i}(t)$, i.e.

$$
f_{\mid \omega}(t, x, v)=e^{-V(x)} \mathcal{M}(v) \sum_{i \in I} \kappa_{i}(t) \mathbb{1}_{\omega_{i}}(x, v)
$$

Since $f$ satisfies the transport equation $(5.20)$ on $\mathbb{R}^{+} \times \omega_{i}$, we infer that $\kappa_{i}$ is constant, so that

$$
f_{\mid \omega}(t, x, v)=f_{\mid \omega}(0, x, v)=e^{-V(x)} \mathcal{M}(v) \sum_{i \in I} \kappa_{i} \mathbb{1}_{\omega_{i}}(x, v)
$$

Using again the transport equation (5.20), we deduce that $\frac{e^{V}}{\mathcal{M}} f(t, x, v)=\frac{e^{V}}{\mathcal{M}} f(0, \cdot) \circ$ $\phi_{-t}(x, v)$. Since there is only one equivalence class for $\approx$, we first deduce that $f=$

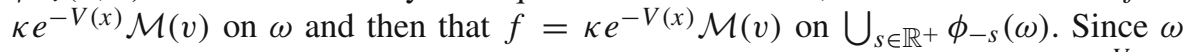
satisfies a.e.i.t. GCC, this is a full measure set and we deduce that $f=\kappa e^{-V} \mathcal{M}$. Since $\int_{\mathbb{T}^{d} \times \mathbb{R}^{d}} f d v d x=0$, necessarily there holds $f=0$.

This concludes the proof of Theorem 2.2.

Remark 5.2 Note that the proof of $($ i. $) \Longrightarrow$ (iii.) relies on the maximum principle for the linear Boltzmann equation (1.1) (i.e. the $\mathcal{L}^{\infty}$ bound). This was in particular useful to prevent loss of mass at infinity for the sequence of solutions under study. This will turn out to be also very useful to overcome another issue in the proof of the analogous theorem in the case of a bounded domain of $\mathbb{R}^{d}$ with specular reflection [27]. 
If $\omega$ satisfies the Geometric Control Condition of Defintion 2.3, we can actually show a slightly stronger property than the Unique Continuation Property. The above proof of $($ ii. $) \Longrightarrow($ i. $)$ in Theorem 2.2 together with the fact that there is a unique equivalence class for $\sim$ under GCC (indeed, $\bigcup_{s \in(0, T)} \phi_{-s}(U)=\mathbb{T}^{d} \times \mathbb{R}^{d}$ is connected) yields the following proposition.

Proposition 5.1 Assume that $(\omega, T)$ satisfies the Geometric Control Condition. If $f \in C_{t}^{0}\left(\mathcal{L}^{2}\right)$ is a solution to

$$
\left\{\begin{array}{l}
\partial_{t} f+v \cdot \nabla_{x} f-\nabla_{x} V \cdot \nabla_{v} f=0 \\
\mathscr{C}(f)=0 \text { on } I \times \omega
\end{array}\right.
$$

where $I$ is an interval of time of length larger than $T$, then $f=\left(\int_{\mathbb{T}^{d} \times \mathbb{R}^{d}} f d v d x\right)$ $e^{-V(x)} \mathcal{M}(v)$.

This will be useful for the proof of Theorem 2.3.

\subsection{Proof of Theorem 2.1}

We start by describing the vector space of stationary solutions of the linear Boltzmann equation (1.1), when the associated set $\omega$ satisfies the a.e.i.t. GCC.

For the sake of readability, we set here

$$
\tilde{\Omega}:=\bigcup_{s \in \mathbb{R}^{+}} \phi_{-s}(\omega) .
$$

We denote $\tilde{\Omega}=\cup_{i \in I} \Omega_{i}$ the partition of $\tilde{\Omega}$ in connected components. We write $\left(\left[\Omega_{j}\right]\right)_{j \in J}$ the equivalence classes for the equivalence relation $\sim$. We denote for all $j \in J$

$$
U_{j}:=\bigcup_{\Omega^{\prime} \in\left[\Omega_{j}\right]} \Omega^{\prime} .
$$

We have the following description of the vector space of stationary solutions of (1.1).

Proposition 5.2 Assume that $\omega$ satisfies the a.e.i.t. GCC. Then a Hilbert basis of the subspace of stationary solutions to the linear Boltzmann equation (1.1) (or, equivalently of $\operatorname{Ker}(A)$, where $A$ is the linear Boltzmann operator defined in (2.11)) is given by the family $\left(f_{j}\right)_{j \in J}$, with

$$
f_{j}=\frac{\mathbb{1}_{U_{j}} e^{-V} \mathcal{M}}{\left\|\mathbb{1}_{U_{j}} e^{-V} \mathcal{M}\right\|_{\mathcal{L}^{2}}} .
$$


In particular, the cardinality of the set of equivalence classes for $\sim$ is equal to the dimension of the vector space of stationary solutions to the linear Boltzmann equation (1.1), i.e.

$$
\operatorname{dim}(\operatorname{Ker}(A))=\sharp(\mathcal{C C}(\tilde{\Omega}) / \sim)=\sharp(\mathcal{C C}(\omega) / \approx)
$$

We can introduce a generalized Unique Continuation Property, as follows.

Definition 5.1 We say that the set $\omega$ satisfies the generalized Unique Continuation Property if the only solutions $f \in C_{t}^{0}\left(\mathcal{L}^{2}\right)$ to

$$
\left\{\begin{array}{l}
\partial_{t} f+v \cdot \nabla_{x} f-\nabla_{x} V \cdot \nabla_{v} f=0 \\
\mathscr{C}(f)=0
\end{array}\right.
$$

are of the form $f=\sum_{j \in J}\left\langle f, f_{j}\right\rangle_{\mathcal{L}^{2}} f_{j}=\sum_{j \in J} \frac{1}{\left\|\mathbb{1}_{U_{j}} e^{-V} \mathcal{M}\right\|_{\mathcal{L}^{2}}}\left(\int_{U_{j}} f d v d x\right) f_{j}$, where $\left(U_{j}\right)_{j \in J}$ is defined in (5.25) and $\left(f_{j}\right)_{j \in J}$ in (5.26).

We can now state the precise version of Theorem 2.1:

Theorem 5.1 We keep the notations of Proposition 5.2. The following statements are equivalent.

(i.) The set $\omega$ satisfies the generalized Unique Continuation Property (see Definition 5.1).

(ii.) The set $\omega$ satisfies the a.e.i.t. GCC.

(iii.) For all $f_{0} \in \mathcal{L}^{2}\left(\mathbb{T}^{d} \times \mathbb{R}^{d}\right)$, denoting by $f(t)$ the unique solution to (1.1) with initial datum $f_{0}$, we have

$$
\left\|f(t)-P f_{0}\right\|_{\mathcal{L}^{2}} \rightarrow_{t \rightarrow+\infty} 0,
$$

where

$$
P f_{0}(x, v)=\sum_{j \in J} \frac{1}{\left\|\mathbb{1}_{U_{j}} e^{-V} \mathcal{M}\right\|_{\mathcal{L}^{2}}}\left(\int_{U_{j}} f_{0} d v d x\right) f_{j},
$$

with $\left(U_{j}\right)_{j \in J}$ defined in (5.25) and $\left(f_{j}\right)_{j \in J}$ defined in (5.26).

(iv.) For all $f_{0} \in \mathcal{L}^{2}\left(\mathbb{T}^{d} \times \mathbb{R}^{d}\right)$, there exists a stationary solution $P f_{0}$ of (1.1) such that we have

$$
\left\|f(t)-P f_{0}\right\|_{\mathcal{L}^{2}} \rightarrow_{t \rightarrow+\infty} 0,
$$

where $f(t)$ is the unique solution to (1.1) with initial datum $f_{0}$.

Note that Theorem 2.2 is a particular case of Theorem 5.1, when there is only one equivalence class for $\sim$ (or equivalently for $\approx$ ).

This section is devoted to the proof of Theorem 5.1 and is organized as follows: in Paragraph 5.2.1, we prove Proposition 5.2. Then, in Paragraph 5.2.2, we prove 
that (iv.) implies (ii.). Finally, in Paragraph 5.2.3, we show that (i.)-(ii.)-(iii.) are equivalent. Since the implication $(i i i) \Longrightarrow(i v$.$) is straightforward, this will conclude$ the proof of Theorem 5.1.

\subsubsection{Proof of Proposition 5.2}

We start by checking that for all $i \in J, f_{j}$ is a stationary solution of (1.1). From Lemma 5.1, we know that for any connected component $\Omega^{\prime}$ of $\tilde{\Omega}$ and any $t \geq 0$, $\phi_{-t}\left(\Omega^{\prime}\right)=\Omega^{\prime}$ up to a set of zero measure. Thus for all $t \geq 0, \phi_{-t}\left(U_{j}\right)=U_{j}$ up to a set of zero measure. The function $f_{j}$ hence cancels the kinetic transport part.

We now check that $f_{j}$ cancels the collision operator, i.e. $\mathscr{C}\left(\mathbb{1}_{U_{i}} e^{-V} \mathcal{M}\right)=0$. We use for this Lemma 4.5.

Denote $\omega=\cup_{i \in I} \omega_{i}$ the partition of $\omega$ in connected components. Let $i \in I$. If $\omega_{i} \cap U_{j}=\emptyset$, then $f_{j}=0$ on $\omega_{i}$. If $\omega_{i} \cap U_{j} \neq \emptyset$, then there exists $\Omega^{\prime} \in\left[\Omega_{j}\right]$ such that $\omega_{i} \cap \Omega^{\prime} \neq \emptyset$. Since $\Omega^{\prime}$ is a connected component of $\bigcup_{s \in \mathbb{R}^{+}} \phi_{-s}(\omega)$, we have $\omega_{i} \subset \Omega^{\prime}$ and thus $f_{j}=\frac{e^{-V(x)} \mathcal{M}(v)}{\left\|\mathbb{1}_{U_{j}} e^{-V} \mathcal{M}\right\|_{\mathcal{L}^{2}}}=\rho_{j}(x) \mathcal{M}(v)$ on $\omega_{i}$, with $\rho_{j}(x)=\frac{e^{-V(x)}}{\left\|\mathbb{1}_{U_{j}} e^{-V} \mathcal{M}\right\|_{\mathcal{L}^{2}}}$.

Assume now that there exist $k, l \in I$ and $x \in \mathbb{T}^{d}$ such that $\omega_{k}(x) \mathcal{R}_{k}^{x} \omega_{l}(x)$. Denote by $\Omega^{\prime} \in\left[\Omega_{j}\right]$, the connected component of $\tilde{\Omega}$ such that $\omega_{l} \subset \Omega^{\prime}$, and $\Omega^{\prime \prime}$ the connected component of $\tilde{\Omega}$ such that $\omega_{k} \subset \Omega^{\prime \prime}$. Note then that $\omega_{k}(x) \mathcal{R}_{k}^{x} \omega_{l}(x)$ implies $\Omega^{\prime} \sim \Omega^{\prime \prime}$. By definition of $f_{j}$, this implies that $\rho_{k}(x)=\rho_{l}(x)$. Therefore, by Lemma 4.5, we infer that the function $f_{j}$ cancels the collision operator. We deduce that $f_{j}$ is a stationary solution of (1.1).

Furthermore, since the supports of the $\left(f_{j}\right)_{j \in J}$ are disjoint, $\left(f_{j}\right)_{j \in J}$ is an orthonormal family of $\mathcal{L}^{2}$.

Finally, let $\varphi$ be a stationary solution of (1.1). Then $\varphi$ satisfies

$$
v \cdot \nabla_{x} \varphi-\nabla_{x} V \cdot \nabla_{v} \varphi=\mathscr{C}(\varphi)
$$

Taking the $\mathcal{L}^{2}$ scalar product with $\varphi$, we deduce that $\mathscr{D}(\varphi)=\langle\mathscr{C}(\varphi), \varphi\rangle_{\mathcal{L}^{2}}=0$, so that by Lemma $4.5, \mathscr{C}(\varphi)=0$. Then, with the same analysis as the proof of (ii.) $\Longrightarrow$ (i.) in Theorem 2.2, we deduce that $\frac{e^{V}}{\mathcal{M}} \varphi$ is constant on each $U_{j}$. Using the fact that $\omega$ satisfies a.e.i.t. GCC, we deduce that we can write

$$
\varphi=\sum_{j \in J} \lambda_{j} \mathbb{1}_{U_{j}} e^{-V} \mathcal{M}(v), \quad \lambda_{j} \in \mathbb{R}
$$

that is

$$
\varphi=\sum_{j \in J}\left\langle\varphi, f_{j}\right\rangle_{\mathcal{L}^{2}} f_{j}
$$

and this concludes the proof. 


\subsubsection{Necessity of the a.e.i.t. Geometric Control Condition}

We prove here that (iv.) implies (ii.) in Theorem 5.1.

We argue by contradiction. Assume that the a.e.i.t. Geometric Control Condition does not hold. Then $\mathcal{A}:=\mathbb{T}^{d} \times \mathbb{R}^{d} \backslash \bigcup_{s \in \mathbb{R}^{+}} \phi_{-s}(\omega)$ has positive Lebesgue measure.

We set

$$
f_{0}(x, v)=\Psi(x) \mathbb{1}_{\mathcal{A}}(x, v) e^{-V(x)} \mathcal{M}(v),
$$

with $\Psi$ to be determined later on. We define

$$
f(t, x, v):=f_{0} \circ \phi_{-t}(x, v)=\Psi \circ \phi_{-t}(x, v) \mathbb{1}_{\phi_{t}(\mathcal{A})}(x, v) e^{-V(x)} \mathcal{M}(v)
$$

which satisfies, by construction,

$$
\partial_{t} f+v \cdot \nabla_{x} f-\nabla_{x} V \cdot \nabla_{v} f=0 .
$$

Note that for all $t \geq 0$, we have $\phi_{t}(\mathcal{A}) \cap \omega=\emptyset$ since $\mathcal{A} \cap \phi_{-t}(\omega)=\emptyset$, which yields $\mathscr{C}(f(t))=0$ for all $t \geq 0$ and thus $f$ is also a solution of (1.1). We now fix $\Psi$ in order to ensure that $f(t)$ is not stationary.

- If $\left(v \cdot \nabla_{x}-\nabla_{x} V \cdot \nabla_{v}\right)\left(\mathbb{1}_{\mathcal{A}}\right) \neq 0$, then we take $\Psi=1$.

- If $\left(v \cdot \nabla_{x}-\nabla_{x} V \cdot \nabla_{v}\right)\left(\mathbb{1}_{\mathcal{A}}\right)=0$, we take $\Psi$ to be a Morse function on $\mathbb{T}^{d}$, so that, in particular, $\Psi$ is smooth and $\nabla \Psi(x) \neq 0$ for almost every $x \in \mathbb{T}^{d}$. Note that with such a function $\Psi$, we have $f_{0} \in \mathcal{L}^{2}$. We compute

$$
\left[v \cdot \nabla_{x} \Psi-\nabla_{x} V \cdot \nabla_{v} \Psi\right] \mathbb{1}_{\mathcal{A}}=\left(v \cdot \nabla_{x} \Psi(x)\right) \mathbb{1}_{\mathcal{A}}(x, v) .
$$

Therefore for almost all $(x, v) \in \mathcal{A}$, this is not null, which shows that $f(t)$ is not stationary.

Finally if there existed a stationary solution $f_{\infty}$ of (1.1) such that

$$
\left\|f(t)-f_{\infty}\right\|_{\mathcal{L}^{2}} \rightarrow t \rightarrow+\infty 0
$$

then since for all $t \geq 0, f(t)$ is supported in $\mathcal{A}$, we also have $f_{\infty}$ supported in $\mathcal{A}$. Thus $f_{\infty}$ cancels the collision operator, i.e. $\mathscr{C}\left(f_{\infty}\right)=0$. We deduce that $f(t)-f_{\infty}$ satisfies

$$
\partial_{t}\left(f-f_{\infty}\right)+v \cdot \nabla_{x}\left(f-f_{\infty}\right)-\nabla_{x} V \cdot \nabla_{v}\left(f-f_{\infty}\right)=0 .
$$

This yields for all $t \geq 0$,

$$
\left\|f(t)-f_{\infty}\right\|_{\mathcal{L}^{2}}=\left\|f_{0}-f_{\infty}\right\|_{\mathcal{L}^{2}}
$$

Moreover, the solution $f$ defined in (5.31) is a non-stationary solution of (5.32) according to the definition of $\Psi$. In conclusion, we have $f_{0} \neq f_{\infty}$. This yields 
$\left\|f_{0}-f_{\infty}\right\|_{\mathcal{L}^{2}}>0$, which, together with (5.34) contradicts (5.33). This concludes the proof of $(i v.) \Longrightarrow($ ii.) in Theorem 5.1.

\subsubsection{End of the Proof of Theorem 5.1}

We have the following key lemma.

Lemma 5.2 Let $f$ be a solution in $C_{t}^{0}\left(\mathcal{L}^{2}\right)$ of (1.1). Then for all $j \in J$,

$$
\frac{d}{d t}\left\langle f, f_{j}\right\rangle_{\mathcal{L}^{2}}=\frac{1}{\left\|\mathbb{1}_{U_{j}} e^{-V} \mathcal{M}\right\|_{\mathcal{L}^{2}}} \frac{d}{d t} \int_{U_{j}} f d v d x=0 .
$$

Proof of Lemma 5.2 Let $f$ be a solution in $C_{t}^{0}\left(\mathcal{L}^{2}\right)$ of $(1.1)$; denote by $f_{0}$ its initial datum. Let $j \in J$. We take the $\mathcal{L}^{2}$ scalar product with $f_{j}$ in (1.1) to obtain

$$
\begin{aligned}
\frac{d}{d t}\left\langle f, f_{j}\right\rangle_{\mathcal{L}^{2}} & =-\left\langle\left(v \cdot \nabla_{x}-\nabla_{x} V \cdot \nabla_{v}\right) f, f_{j}\right\rangle_{\mathcal{L}^{2}}+\left\langle\mathscr{C}(f), f_{j}\right\rangle_{\mathcal{L}^{2}} \\
& =\left\langle f,\left(v \cdot \nabla_{x}-\nabla_{x} V \cdot \nabla_{v}\right) f_{j}\right\rangle_{\mathcal{L}^{2}}+\left\langle f, \mathscr{C}^{*}\left(f_{j}\right)\right\rangle_{\mathcal{L}^{2}}
\end{aligned}
$$

where $\mathscr{C}^{*}$ is the collision operator of collision kernel defined by

$$
\mathscr{C}^{*}(g)(x, v)=\int_{\mathbb{R}^{d}}\left[\tilde{k}\left(x, v, v^{\prime}\right) \mathcal{M}(v) g\left(v^{\prime}\right)-\tilde{k}\left(x, v^{\prime}, v\right) \mathcal{M}\left(v^{\prime}\right) g(v)\right] d v^{\prime}
$$

with $\tilde{k}\left(x, v, v^{\prime}\right)=\frac{k\left(x, v, v^{\prime}\right)}{\mathcal{M}\left(v^{\prime}\right)}$. This follows from Property $\mathbf{A} 2$ satisfied by $k$.

We then use the following two facts.

(1) We have $\left(v \cdot \nabla_{x}-\nabla_{x} V \cdot \nabla_{v}\right) f_{j}=0$ (see the proof of Proposition 5.2).

(2) We have $\mathscr{C}^{*}\left(f_{j}\right)=0$. This follows from Property $\mathbf{A 2}$, the fact that $\mathscr{C}\left(f_{j}\right)=0$ (see again the proof of Proposition 5.2) and Lemma 4.5.

We conclude that $\frac{d}{d t}\left\langle f, f_{j}\right\rangle_{\mathcal{L}^{2}}=0$.

We therefore infer that if $f(t)$ satisfies (1.1) with an initial datum $f_{0}$, then for all $t \geq 0$

$$
\int_{U_{j}} f(t) d v d x=\int_{U_{j}} f_{0} d v d x
$$

Equipped with this result, we can prove the equivalence between (i.)-(ii.)-(iii.) exactly as for Theorem 2.2, with only minor adaptations. The details are left to the reader.

\section{Application to Particular Classes of Collision Kernels}

In this section, we introduce different classes of collision kernels to illustrate the main results of the previous sections. We then draw consequences of the additional assumptions made in these examples. 
E3. Let $k$ be a collision kernel verifying A1-A3. Let $\omega$ be the set where collisions are effective, defined in (2.1). We moreover require that for all $(x, v),\left(x, v^{\prime}\right) \in \omega$, there exist $N \in \mathbb{N}^{*}$ and a "chain" $v_{1}, \cdots, v_{N} \in \mathbb{R}^{d}$ such that the following hold.

- For all $i, 1 \leq i \leq N,\left(x, v_{i}\right) \in \omega$.

- The points $(x, v)$ and $\left(x, v_{1}\right)$ belong to the same connected component of $\omega$.

- The points $\left(x, v^{\prime}\right)$ and $\left(x, v_{N}\right)$ belong to the same connected component of $\omega$.

- For all $i, 1 \leq i \leq N-1$, we have

$$
k\left(x, v_{i}, v_{i+1}\right)>0 \text { or } k\left(x, v_{i+1}, v_{i}\right)>0 .
$$

As a subclass of $\mathbf{E 3}$, we have

E3'. Let $k$ be a collision kernel verifying A1-A3. We require that for all $y \in p_{x}(\omega)$ (where $p_{x}(\omega)$ is the projection of $\omega$ on $\mathbb{T}^{d}$ ), the set $p_{x}^{-1}(\{y\})$ is included in one single connected component of $\omega$.

A trivial subclass of $\mathbf{E 3}^{\prime}$ is the case where $\omega$ is connected. Another subclass of E3' is given in the following example.

E3". Let $k$ be a collision kernel verifying A1-A3. We require that

$$
\omega=\omega_{x} \times \mathbb{R}^{d},
$$

where $\omega_{x}$ is an open subset of $\mathbb{T}^{d}$.

Remark that E2 is a subclass of E3".

In what follows, we explain the interest of these classes of collision kernels regarding the geometric definitions introduced before.

\subsection{The Case of Collision Kernels in The Class E3}

The interest of $\mathbf{E 3}$ lies in the simple description of the kernel of the associated collision operator $\mathscr{C}$.

Using Lemma 4.5 and the "chain" in the definition of a collision kernel in $\mathbf{E 3}$, we deduce the following result.

Lemma 6.1 Let $k$ be a collision kernel in the class E3. Let $T \in(0,+\infty]$ and assume that $f \in L^{2}\left(0, T ; \mathcal{L}^{2}\right)$ satisfies $\mathscr{C}(f(t))=0$ for almost every $t \in[0, T]$. Then there is a function $\rho \in L^{2}\left(0, T ; L^{2}\left(\mathbb{T}^{d}\right)\right)$ such that

$$
f=\rho(t, x) \mathcal{M}(v) \text { on }[0, T] \times \omega .
$$

Reciprocally, any function $f$ satisfying this property satisfies $\mathscr{C}(f)=0$.

In other words, the kernel of the associated collision operator is equal to the set of functions which are Maxwellians on $\omega$ :

$$
\operatorname{Ker}(\mathscr{C})=\left\{f \in \mathcal{L}^{2}, f_{\mid \omega}=\rho(x) \mathcal{M}(v)\right\} .
$$

We recall that this property, which is usual in the non degenerate case $\omega=\mathbb{T}^{d} \times \mathbb{R}^{d}$, is not true in general for collision kernels satisfying merely A1, A2 and A3. 
This allows us to reformulate in a very simple way the Unique Continuation Property for collision kernels in E3.

Lemma 6.2 Let $k$ be a collision kernel in the class E3. Then the set $\omega$ satisfies the Unique Continuation Property if and only if the only solution $f \in C_{t}^{0}\left(\mathcal{L}^{2}\right)$ to

$$
\left\{\begin{array}{l}
\partial_{t} f+v \cdot \nabla_{x} f-\nabla_{x} V \cdot \nabla_{v} f=0 \\
f=\rho(t, x) \mathcal{M}(v) \text { on } \mathbb{R}^{+} \times \omega
\end{array}\right.
$$

is $f=\left(\int_{\mathbb{T}^{d} \times \mathbb{R}^{d}} f d v d x\right) e^{-V(x)} \mathcal{M}(v)$.

Conversely, using again Lemma 4.5, we have the following result.

Lemma 6.3 Let $k$ be a collision kernel satisfying A1-A3. If any function $f \in \mathcal{L}^{2}$ cancelling the collision operator has its restriction to $\omega$ satisfying

$$
f_{\mid \omega}=\mathbb{1}_{\omega} \rho(x) \mathcal{M}(v)
$$

for some $\rho \in L^{2}\left(\mathbb{T}^{d}\right)$, then $k$ necessarily belongs to the class $\mathbf{E} 3$.

Therefore, E3 is the largest class of collision kernels such that the kernel of the associated collision operator is equal to the set of functions which are Maxwellians on $\omega$, i.e. for which (6.1) holds.

\subsection{The Case of Collision Kernels in the Class E3'}

To explain the interest of E3', let us introduce now another geometric condition:

(iv.) The set $\omega$ satisfies the a.e.i.t. GCC and $\bigcup_{s \in \mathbb{R}^{+}} \phi_{-s}(\omega)$ is connected.

This condition is compared to other geometric conditions in Appendix 3.2. It has to be confronted to the geometric condition of Theorem 2.2 (rephrased using Lemma 3.1):

(ii.) The set $\omega$ satisfies the a.e.i.t. GCC and there is only one equivalence class for $\sim$.

In what follows, we shall adopt the notations of Theorem 2.2. It is clear that ( $i v$.) implies (ii.), as ( $i v$.) means that there is a single equivalence class for an equivalence relation defined as in Definition 2.9 with $\mathcal{R}_{\phi}$ only. However, it is false in general that items (i.)-(iii.) in Theorem 2.2 and (iv.) above are equivalent; see Proposition 6.1 below for an example of collision kernel such that (i.)-(iii.) are satisfied, but not (iv.).

Proposition 6.1 For $V=0$, there exists a collision kernel $k$ in the class $\mathbf{E} 1$ and $\mathbf{E 3 ,}$ for which (ii) holds, but not (iv).

Proof of Proposition 6.1 Consider $(x, v) \in \mathbb{T} \times \mathbb{R}$ (a similar example can be constructed in higher dimension as well). We take a function $\varphi \in C^{0}(\mathbb{R})$, such that $\varphi(0)=0$ and $\varphi(v)>0$ for all $v \in \mathbb{R} \backslash\{0\}$. Define

$$
k\left(x, v, v^{\prime}\right)=\varphi(v) \varphi\left(v^{\prime}\right) \mathcal{M}\left(v^{\prime}\right), \quad \text { i.e. } \quad \tilde{k}\left(x, v, v^{\prime}\right)=\varphi(v) \varphi\left(v^{\prime}\right)
$$


By construction, $\mathbf{A 1}$ and $\mathbf{A} \mathbf{3}$ are satisfied and we notice that $\tilde{k}$ is symmetric (so that $k$ is in $\mathbf{E 1}$ ). We can also readily check that $k$ is in $\mathbf{E 3}$ (with $N=2$ ).

We have

$$
\omega:=\left\{\mathbb{T} \times \mathbb{R}_{*}^{-}\right\} \cup\left\{\mathbb{T} \times \mathbb{R}_{*}^{+}\right\}
$$

and so

$$
\bigcup_{t \geq 0} \phi_{-t}(\omega)=\left\{\mathbb{T} \times \mathbb{R}_{*}^{-}\right\} \cup\left\{\mathbb{T} \times \mathbb{R}_{*}^{+}\right\}
$$

from which we deduce that a.e.i.t. GCC is satisfied, but $\cup_{t \geq 0} \phi_{-t}(\omega)$ is not connected.

On the other hand, the set $\omega$ satisfies the unique continuation property. Indeed, let $f$ satisfying

$$
\partial_{t} f+v \cdot \nabla_{x} f=0, \quad \forall(x, v) \in \mathbb{T} \times \mathbb{R}
$$

and $\mathscr{C}(f)=0$. Using Lemma 6.1 and the definition of $k$, we deduce that $f=$ $\rho(t, x) \mathcal{M}(v)$ on $\mathbb{R}^{+} \times \mathbb{T} \times \mathbb{R} \backslash\{0\}$, and thus almost everywhere in $\mathbb{R}^{+} \times \mathbb{T} \times \mathbb{R}$.

As $f$ satisfies the transport equation (6.2), this implies that $f=C \mathcal{M}(v)$ for some $C>0$ and we conclude that the unique continuation property holds.

Therefore, by Theorem 2.2, we deduce that (ii.) holds.

However, when restricting to collision kernels in the class E3', Conditions (ii.) and (iv.) become equivalent.

Proposition 6.2 Let $k$ be a collision kernel in the class E3'. Then (i.)-(iv.) are equivalent.

Proof of Proposition 6.2 We consider $k$ a collision kernel in the class E3'. Assume that (ii.) holds. The aim is to prove that ( $i v$.) holds. By contradiction, assume that there are at least two connected components $\Omega_{1}, \Omega_{2}$ of $\bigcup_{t>0} \phi_{-t}(\omega)$.

By (ii.), $\Omega_{1}$ and $\Omega_{2}$ belong to the same equivalence class for $\sim$. Thus, there exist $x, v_{1}, v_{2}$ with $\left(x, v_{1}\right) \in \Omega_{1},\left(x, v_{2}\right) \in \Omega_{2}$ and

$$
k\left(x, v_{1}, v_{2}\right)>0 \text { or } k\left(x, v_{2}, v_{1}\right)>0 .
$$

But since $k$ is in the class E3', the set $p_{x}^{-1}(\{x\})$ is included in one connected component of $\omega$. Thus we cannot have $\left(x, v_{1}\right) \in \Omega_{1}$ and $\left(x, v_{2}\right) \in \Omega_{2}$. This is a contradiction and this concludes the proof.

More generally, we observe that for collision kernels in E3', the equivalence classes for $\sim$ are exactly the connected components of $\bigcup_{t \geq 0} \phi_{-t}(\omega)$. Thus Theorem 5.1 can be reformulated as follows.

Corollary 6.1 Let $k$ be a collision kernel in the class E3'. The following statements are equivalent. 
(1) The set $\omega$ satisfies the generalized Unique Continuation Property.

(2) The set $\omega$ satisfies the a.e.i.t. GCC.

(3) Let $\left(\Omega_{i}\right)_{i \in I}$ be the connected components of $\cup_{t \geq 0} \phi_{-t}(\omega)$. For all $f_{0} \in \mathcal{L}^{2}\left(\mathbb{T}^{d} \times\right.$ $\left.\mathbb{R}^{d}\right)$, denote by $f(t)$ the unique solution to (1.1) with initial datum $f_{0}$. We have

$$
\left\|f(t)-P f_{0}\right\|_{\mathcal{L}^{2}} \rightarrow_{t \rightarrow+\infty} 0
$$

where

$$
P f_{0}=\sum_{i \in I} \frac{1}{\left\|\mathbb{1}_{\Omega_{i}} e^{-V} \mathcal{M}\right\|_{\mathcal{L}^{2}}}\left(\int_{\Omega_{i}} f_{0} d v d x\right) g_{j}
$$

with for all $i \in I$,

$$
g_{i}=\frac{\mathbb{1}_{\Omega_{i}} e^{-V} \mathcal{M}}{\left\|\mathbb{1}_{\Omega_{i}} e^{-V} \mathcal{M}\right\|_{\mathcal{L}^{2}}} .
$$

We close this section by exhibiting an example of collision kernel in E3', for which Corollary 6.1, and thus Theorem 5.1, are relevant.

We restrict ourselves to the case $\mathbb{T} \times \mathbb{R}$ (this can be easily adapted to higher dimensions). We consider the free transport case, i.e. $V=0$. We identify $\mathbb{T}$ to $[-1 / 2,1 / 2)$. Consider $\alpha \in C^{0}(\mathbb{T})$ supported in $[-1 / 2,0)$ and $\beta \in C^{0}(\mathbb{T})$ supported in $[0,1 / 2)$ that do not vanish identically.

Let $\varphi \in L^{\infty} \cap C^{0}(\mathbb{R})$ such that $\varphi>0$ on $\mathbb{R}_{*}^{-}$and $\varphi=0$ on $\mathbb{R}^{+}$. Likewise, let $\Psi \in L^{\infty} \cap C^{0}(\mathbb{R})$ such that $\Psi>0$ on $\mathbb{R}_{*}^{+}$and $\Psi=0$ on $\mathbb{R}^{-}$. We define the collision kernel

$$
k\left(x, v, v^{\prime}\right):=\left[\alpha(x) \varphi(v) \varphi\left(v^{\prime}\right)+\beta(x) \Psi(v) \Psi\left(v^{\prime}\right)\right] \mathcal{M}\left(v^{\prime}\right) .
$$

Note that $\tilde{k}\left(x, v, v^{\prime}\right)=\alpha(x) \varphi(v) \varphi\left(v^{\prime}\right)+\beta(x) \Psi(v) \Psi\left(v^{\prime}\right)$ is symmetric in $v$ and $v^{\prime}$, and belongs to $L^{\infty}$. Thus $k$ is in the class E1. Furthermore, we readily check $k$ is in the class E3'.

Moreover, we have $\omega=\left\{\{\alpha>0\} \times \mathbb{R}_{*}^{-}\right\} \cup\left\{\{\beta>0\} \times \mathbb{R}_{*}^{+}\right\}$, and

$$
\bigcup_{s \in \mathbb{R}^{+}} \phi_{-s}(\omega)=\left\{\mathbb{T} \times \mathbb{R}_{*}^{-}\right\} \cup\left\{\mathbb{T} \times \mathbb{R}_{*}^{+}\right\}
$$

Thus $\omega$ satisfies the a.e.i.t. GCC but $\bigcup_{s \in \mathbb{R}^{+}} \phi_{-s}(\omega)$ is not connected.

The basis of the subspace of stationary solutions of (1.1) is given by $\left(f_{j}\right)_{j=1,2}$ with

$$
f_{1}=\frac{\mathbb{1}_{\mathbb{T} \times \mathbb{R}_{*}^{-}} e^{-V} \mathcal{M}}{\left\|\mathbb{1}_{\mathbb{T} \times \mathbb{R}_{*}^{-}} e^{-V} \mathcal{M}\right\|_{\mathcal{L}^{2}}}, \quad f_{2}=\frac{\mathbb{1}_{\mathbb{T} \times \mathbb{R}_{*}^{+}} e^{-V} \mathcal{M}}{\left\|\mathbb{1}_{\mathbb{T} \times \mathbb{R}_{*}^{+}} e^{-V} \mathcal{M}\right\|_{\mathcal{L}^{2}}} .
$$




\subsection{The Case of Collision Kernels in the Class E3"}

We finally study collision kernels in the class E3". Because of the remarkable properties of the geodesic flow on the torus $\mathbb{T}^{d}$, the following holds.

Lemma 6.4 Suppose that $V=0$ and that the collision kernel belongs to the class E3". Then $\omega$ satisfies a.e.i.t. GCC.

Proof of Lemma 6.4 Define $T_{v}^{t}: x \mapsto x+t v$; then $\left(T_{v}^{t} x\right)_{t \geq 0}$ is dense in $\mathbb{T}^{d}$ for almost every $(x, v) \in \mathbb{T}^{d} \times \mathbb{R}^{d}$ (with respect to the Lebesgue measure). This proves the lemma.

We deduce a proof of Proposition 3.1.

Proof of Proposition 3.1 Take $\omega_{x}^{0}$ a connected component of $\omega_{x}$. According to Lemma 6.4, $\omega_{x}^{0} \times \mathbb{R}^{d}$ satisfies a.e.i.t. GCC. The result then follows from Proposition 13.1, (i.) $\Rightarrow$ (iii.), and Theorem 2.2.

\section{Characterization of Exponential Convergence}

Let us first briefly recall why $C^{-}(\infty)$ is well-defined (see [32]). We can first define for all $T>0$,

$$
C^{-}(T):=\inf _{(x, v) \in \mathbb{T}^{d} \times \mathbb{R}^{d}} \frac{1}{T} \int_{0}^{T}\left(\int_{\mathbb{R}^{d}} k\left(\phi_{t}(x, v), v^{\prime}\right) d v^{\prime}\right) d t,
$$

which is a continuous nonnegative function since $k$ is. We then remark that the function $T \mapsto-T C^{-}(T)$ is subadditive. This entails that $C^{-}(\infty)=\lim _{T \rightarrow+\infty} C^{-}(T)$ exists.

In this section, we assume that $k$ satisfies A3' and provide the proof of Theorem 2.3. To this end, we first prove that (a.) and (b.) are equivalent, and finally that (c.) implies (a.). This will conclude the proof, noticing that (b.) implies (c.) is straightforward. One can also readily check that in the proof of (a.) implies (b.), the assumption A3' is not used.

\subsection{Proof of Theorem 2.3, (a.) $\Longleftrightarrow$ (b.)}

Since the equation (1.1) is linear, if $f(t)$ satisfies (1.1) then

$$
g(t):=f(t)-\left(\int_{\mathbb{T}^{d} \times \mathbb{R}^{d}} f(0) d v d x\right) e^{-V} \mathcal{M},
$$

is still a solution to (1.1). Thus we can deal only with initial data which have zero average.

By conservation of the mass, the Boltzmann equation (1.1) is well-posed in the space

$$
\mathcal{L}_{0}^{2}:=\left\{f \in \mathcal{L}^{2}, \int f d v d x=0\right\}
$$


and we can use Lemma 11.1 for solutions in $\mathcal{L}_{0}^{2}$, which yields that $(b$.) is equivalent to

(b'.) There exists $T>0$ and $K>0$ such that for all $f_{0} \in \mathcal{L}_{0}^{2}$, the associated solution $f$ to (1.1) satisfies

$$
K \int_{0}^{T} \mathscr{D}(f(t)) d t \geq\left\|f_{0}\right\|_{\mathcal{L}^{2}}^{2}
$$

We first prove that $(a$.$) implies \left(b^{\prime}.\right)$, then that $\left(b^{\prime}.\right)$ implies $(a$.$) .$

$(a.) \Longrightarrow\left(b^{\prime}\right.$.) Assume that $(a$.) holds.

We argue by contradiction. Denying $\left(b^{\prime}\right.$.) is equivalent to assuming for all $T>0$ and all $C>0$, the existence of $g_{0}^{C, T} \in \mathcal{L}_{0}^{2}$, such that

$$
C \int_{0}^{T} \mathscr{D}\left(g^{C, T}(t)\right) d t<\left\|g_{0}^{C, T}\right\|_{\mathcal{L}^{2}}^{2},
$$

where $g^{C, T}(t)$ is the unique solution to (1.1) with initial datum $g_{0}^{C, T}$. Taking $T=n$ and $C=n$, this yields for all $n \in \mathbb{N}^{*}$, the existence of $g_{0, n} \in \mathcal{L}_{0}^{2}$ such that

$$
\int_{0}^{n} \mathscr{D}\left(g_{n}(t)\right) d t<\frac{1}{n}\left\|g_{0, n}\right\|_{\mathcal{L}^{2}}^{2}
$$

where $g_{n}(t)$ is the unique solution to (1.1) with initial datum $g_{0, n}$. Furthermore, by linearity of (1.1), we can normalize the initial data so that for all $n \in \mathbb{N}^{*}$,

$$
\left\|g_{0, n}\right\|_{\mathcal{L}^{2}}=1
$$

Recall that by (4.5), we have, for all $t \geq 0$,

$$
\left\|g_{n}(t)\right\|_{\mathcal{L}^{2}}^{2}-\left\|g_{0, n}\right\|_{\mathcal{L}^{2}}^{2}=-\int_{0}^{t} \mathscr{D}\left(g_{n}(s)\right) d s .
$$

In particular, the sequence $\left(g_{n}\right)_{n \in \mathbb{N}^{*}}$ is uniformly bounded in $L_{t}^{\infty} \mathcal{L}^{2}$; thus, up to some extraction, we can assume that $g_{n} \rightarrow g$ weakly in $L_{t, l o c}^{2} \mathcal{L}^{2}$. Let us prove that $g=0$. By linearity of (1.1), $g$ still satisfies (1.1) since $g_{n}$ does.

Note also that by conservation of the mass, for all $n \in \mathbb{N}$ and all $t \geq 0$, we have

$$
\int g_{n}(t) d v d x=\int g_{0, n} d v d x=0 .
$$

Take $T^{\prime}>0$ such that $\left(\omega, T^{\prime}\right)$ satisfies GCC. By (7.1), we have $\int_{0}^{T^{\prime}} \mathscr{D}\left(g_{n}(t)\right) d t \rightarrow$ 0 and therefore by Lemma 4.2, we deduce

$$
\|\mathscr{D}(g)\|_{L^{1}\left(0, T^{\prime}\right)} \leq \liminf _{n \rightarrow+\infty}\left\|\mathscr{D}\left(g_{n}\right)\right\|_{L^{1}\left(0, T^{\prime}\right)}=0
$$


As a consequence, by weak coercivity (see Lemma 4.5), we infer that $\mathscr{C}(h)=0$ on $\left[0, T^{\prime}\right]$, and therefore $h$ satisfies the kinetic transport equation (2.6). The Unique Continuation Property of Proposition 5.1 then implies that

$$
g=\left(\int_{\mathbb{T}^{d} \times \mathbb{R}^{d}} g d v d x\right) e^{-V(x)} \mathcal{M}(v) .
$$

Since $g_{n} \rightarrow g$ weakly in $L_{t, l o c}^{2} \mathcal{L}^{2}$, using (7.4), we obtain in particular that

$$
\int_{0}^{T^{\prime}}\left(\int g d v d x\right) d t=0
$$

Since $\int_{0}^{T^{\prime}}\left(\int g d v d x\right) d t=T^{\prime}\left(\int g(0) d v d x\right)$, we deduce that $\int g d v d x=0$, so that $g=0$. Therefore, this leads to $g=0$.

Now, let us study the sequence of defect measures $v_{n}:=\left|g_{n}\right|^{2}$ and $\nu_{0, n}:=\left|g_{0, n}\right|^{2}$. Consider the equation (1.1) satisfied by $g_{n}$ and multiply it by $g_{n}$ to get:

$$
\begin{aligned}
\partial_{t} v_{n} & +v \cdot \nabla_{x} v_{n}-\nabla_{x} V \cdot \nabla_{v} v_{n} \\
& =2\left(\int_{\mathbb{R}^{d}} k\left(x, v^{\prime}, v\right) g_{n}\left(v^{\prime}\right) d v^{\prime}\right) g_{n}-2\left(\int_{\mathbb{R}^{d}} k\left(x, v, v^{\prime}\right) d v^{\prime}\right) v_{n} .
\end{aligned}
$$

By Duhamel's formula, we infer

$$
\begin{aligned}
& v_{n}(t, x, v)=e^{-2 \int_{0}^{t} \int_{\mathbb{R}^{d}} k\left(\phi_{s-t}(x, v), v^{\prime}\right) d v^{\prime} d s} v_{0, n}\left(\phi_{-t}(x, v)\right) \\
& \quad+\int_{0}^{t} 2 \mathscr{C}^{+}\left(g_{n}(s)\right)\left(\phi_{s-t}(x, v)\right) g_{n}\left(s, \phi_{s-t}(x, v)\right) e^{-2 \int_{s}^{t} \int_{\mathbb{R}^{d}} k\left(\phi_{\tau-t}(x, v), v^{\prime}\right) d v^{\prime} d \tau} d s,
\end{aligned}
$$

where, more explicitely, we have

$\mathscr{C}^{+}\left(g_{n}(s)\right)\left(\phi_{s-t}(x, v)\right)=\int_{\mathbb{R}^{d}} k\left(X_{s-t}(x, v), v^{\prime}, \Xi_{s-t}(x, v)\right) g_{n}\left(s, X_{s-t}(x, v), v^{\prime}\right) d v^{\prime}$.

As a consequence, we get for all $t \geq 0$,

$$
\begin{aligned}
& \left\|v_{n}(t)\right\|_{\mathcal{L}^{1}} \leq A_{n}(t)+B_{n}(t) \text { with } \\
& A_{n}(t)=\int_{\mathbb{T}^{d} \times \mathbb{R}^{d}} e^{-2 \int_{0}^{t} \int_{\mathbb{R}^{d}} k\left(\phi_{s-t}(x, v), v^{\prime}\right) d v^{\prime} d s} v_{0, n}\left(\phi_{-t}(x, v)\right) \frac{e^{V(x)}}{\mathcal{M}(v)} d v d x \\
& B_{n}(t)=\int_{\mathbb{T}^{d} \times \mathbb{R}^{d}}\left(\int_{0}^{t} 2\left|\mathscr{C}^{+}\left(g_{n}(s)\right)\left(\phi_{s-t}(x, v)\right)\right|\left|g_{n}\left(s, \phi_{s-t}(x, v)\right)\right|\right. \\
& \left.\quad \times e^{-2 \int_{s}^{t} \int_{\mathbb{R}^{d}} k\left(\phi_{\tau-t}(x, v), v^{\prime}\right) d v^{\prime} d \tau} d s\right) \frac{e^{V(x)}}{\mathcal{M}(v)} d v d x
\end{aligned}
$$


By definition of $C^{-}(\infty)$, there exists $T_{0}>0$ large enough such that for all $t \geq$ $T_{0}, C^{-}(t) \geq C^{-}(\infty) / 2>0$. We infer, after the change of variables $\phi_{-t}(x, v) \mapsto$ $(x, v)$, which has unit Jacobian (recall also that the hamiltonian is left invariant by this transform), that for all $t \geq T_{0}$,

$$
\begin{aligned}
A_{n}(t) & =\int_{\mathbb{T}^{d} \times \mathbb{R}^{d}} e^{-2 \int_{0}^{t} \int_{\mathbb{R}^{d}} k\left(\phi_{s}(x, v), v^{\prime}\right) d v^{\prime} d s} v_{0, n}(x, v) \frac{e^{V(x)}}{\mathcal{M}(v)} d v d x \\
& \leq e^{-t C^{-}(t)}\left\|v_{0, n}\right\|_{\mathcal{L}^{1}} \leq e^{-t C^{-}(\infty) / 2}\left\|v_{0, n}\right\|_{\mathcal{L}^{1}}
\end{aligned}
$$

and thus we can choose $T_{1} \geq T_{0}$ large enough such that (we recall that $\left\|v_{0, n}\right\|_{\mathcal{L}^{1}}=1$ )

$$
A_{n}\left(T_{1}\right) \leq \frac{1}{4}\left\|v_{0, n}\right\|_{\mathcal{L}^{1}}=\frac{1}{4}
$$

which tackles the first term in (7.7).

We now study the second term in (7.7) on the time interval $\left(0, T_{1}\right)$. Since $g_{n} \rightarrow 0$, by the averaging lemma of Corollary 12.2 , we deduce that

$$
\mathscr{C}^{+}\left(g_{n}(s)\right)(x, v)=\left(\int_{\mathbb{R}^{d}} k\left(x, v^{\prime}, v\right) g_{n}\left(s, x, v^{\prime}\right) d v^{\prime}\right) \rightarrow 0 \quad \text { in } \quad L^{2}\left(0, T_{1} ; \mathcal{L}^{2}\right) .
$$

Hence, by the weak/strong convergence principle,

$$
\begin{aligned}
\mathscr{C}^{+} & \left(g_{n}(s)\right)(x, v) g_{n}(s, x, v) \\
& =\left(\int_{\mathbb{R}^{d}} k\left(x, v^{\prime}, v\right) g_{n}\left(s, x, v^{\prime}\right) d v^{\prime}\right) g_{n} \rightarrow 0, \quad \text { in } \quad L^{1}\left(0, T_{1} ; \mathcal{L}^{1}\right) .
\end{aligned}
$$

We may now estimate the second term in (7.7) by

$$
B_{n}(t) \leq \int_{\mathbb{T}^{d} \times \mathbb{R}^{d}}\left(\int_{0}^{t} 2\left|\mathscr{C}^{+}\left(g_{n}(s)\right)\left(\phi_{s-t}(x, v)\right)\right|\left|g_{n}\left(s, \phi_{s-t}(x, v)\right)\right| d s\right) \frac{e^{V(x)}}{\mathcal{M}(v)} d v d x
$$

change variable $t-s \mapsto s$ in the time integral, apply the Fubini theorem, and finally use the change of variables $\phi_{s}(x, v) \mapsto(x, v)$, which has unit Jacobian, to obtain, for $t=T_{1}$,

$$
B_{n}\left(T_{1}\right) \leq \int_{0}^{T_{1}} \int_{\mathbb{T}^{d} \times \mathbb{R}^{d}} 2\left|\mathscr{C}^{+}\left(g_{n}(s)\right)(x, v)\right|\left|g_{n}(s, x, v)\right| \frac{e^{V(x)}}{\mathcal{M}(v)} d v d x d s \rightarrow 0,
$$

according to (7.8). Thus, coming back to (7.7), for $n$ large enough, we finally obtain

$$
\left\|v_{n}\left(T_{1}\right)\right\|_{\mathcal{L}^{1}} \leq \frac{1}{2} .
$$


But integrating with respect to time (7.3) and using (7.2)-(7.1), we also have

$$
\begin{aligned}
\left\|v_{n}\left(T_{1}\right)\right\|_{\mathcal{L}^{1}} & =1-\int_{0}^{T_{1}} \mathscr{D}\left(g_{n}(s)\right) d s \\
& \geq \frac{3}{4} \quad \text { for } n \text { large enough, }
\end{aligned}
$$

which is a contradiction with (7.9). This concludes the proof of $(a.) \Longrightarrow\left(b^{\prime}.\right)$.

$\left(b^{\prime}.\right) \Longrightarrow\left(a\right.$.) We show that if $\left(a\right.$.) does not hold (i.e. $\left.C^{-}(\infty)=0\right)$, then $\left(b^{\prime}\right.$.) does not either. Assume that $(a$.) does not hold. The goal is to show that for all $T>0$, for all $\varepsilon>0$, there exists $g_{0, \varepsilon} \in \mathcal{L}_{0}^{2}$ such that

$$
\left\|g_{0, \varepsilon}\right\|_{\mathcal{L}^{2}}=1, \quad \int_{0}^{T} \mathscr{D}\left(g_{\varepsilon}\right)(t) d t<\varepsilon
$$

where $g_{\varepsilon}$ is the solution to (1.1) with initial datum $g_{0, \varepsilon}$.

Fix $T>0$ and $\varepsilon>0$. Since $C^{-}(\infty)=0$, there exists $\left(x_{0}, v_{0}\right) \in \mathbb{T}^{d} \times \mathbb{R}^{d}$, such that

$$
\int_{0}^{T} \int_{\mathbb{R}^{d}} k\left(\phi_{t}\left(x_{0}, v_{0}\right), v^{\prime}\right) d v^{\prime} d t \frac{e^{V\left(x_{0}\right)}}{\mathcal{M}\left(v_{0}\right)}<\varepsilon / 3
$$

Let $\chi$ be a smooth compactly supported cutoff function defined from $\mathbb{R}^{+}$to $\mathbb{R}$ such that $\chi \equiv 1$ on $[0,1]$ and $\chi \equiv 0$ on $[2, \infty)$ and such that $\int_{\mathbb{R}^{+}} \chi(r) r^{d-1} d r=0$. Consider

$$
\tilde{g}_{0, n}=\chi\left(n\left|x-x_{0}\right|\right) \chi\left(n\left|v-v_{0}\right|\right) .
$$

Then notice that there is $\alpha>0$ independent of $n$ such that

$$
\left\|\tilde{g}_{0, n}\right\|_{\mathcal{L}^{2}}^{2}=n^{-2 d} \alpha
$$

The function $g_{0, n}:=\frac{n^{d}}{\alpha} \tilde{g}_{0, n}$ is thus normalized in $\mathcal{L}^{2}$. Note that by construction,

$$
\int g_{0, n} d v d x=\frac{n^{d}}{\alpha}\left(\int \chi\left(n\left|x-x_{0}\right|\right) d x\right)\left(\int \chi\left(n\left|v-v_{0}\right|\right) d v\right)=0,
$$

and thus $g_{0, n} \in \mathcal{L}_{0}^{2}$.

We call $g_{n}$ the solution to (1.1) with initial datum $g_{0, n}$. By construction, we observe that $g_{0, n} \rightarrow 0$ weakly in $\mathcal{L}^{2}$ and we deduce that $g_{n} \rightarrow 0$ weakly in $L_{t, l o c}^{2} \mathcal{L}^{2}$. As in the previous proofs, by the averaging lemma of Corollary 12.2, this implies that

$$
\mathscr{C}^{+}\left(g_{n}(t)\right)(x, v)=\int k\left(x, v^{\prime}, v\right) g_{n}\left(t, x, v^{\prime}\right) d v^{\prime} \rightarrow 0, \text { strongly in } L_{t, l o c}^{2} \mathcal{L}^{2}
$$


Now, consider $v_{n}:=\left|g_{n}\right|^{2}$. By construction, we have, in the sense of distributions,

$$
v_{n}(0) \rightarrow \delta_{x=x_{0}, v=v_{0}}
$$

where $\delta$ denotes as usual the Dirac measure. As in (7.6), we have the Duhamel's formula

$$
\begin{aligned}
& v_{n}(t, x, v)=e^{-2 \int_{0}^{t} \int_{\mathbb{R}^{d}} k\left(\phi_{s-t}(x, v), v^{\prime}\right) d v^{\prime} d s} v_{0, n}\left(\phi_{-t}(x, v)\right) \\
& \quad+\int_{0}^{t} 2 \mathscr{C}^{+}\left(g_{n}(s)\right)\left(\phi_{s-t}(x, v)\right) g_{n}\left(s, \phi_{s-t}(x, v)\right) e^{-2 \int_{s}^{t} \int_{\mathbb{R}^{d}} k\left(\phi_{\tau-t}(x, v), v^{\prime}\right) d v^{\prime} d \tau} d s,
\end{aligned}
$$

where, more explicitely, we have $\mathscr{C}^{+}\left(g_{n}(s)\right)\left(\phi_{s-t}(x, v)\right)=\int_{\mathbb{R}^{d}} k\left(X_{s-t}(x, v), v^{\prime}, \Xi_{s-t}(x, v)\right) g_{n}\left(s, X_{s-t}(x, v), v^{\prime}\right) d v^{\prime}$.

We now want to prove that $\int_{0}^{T} \mathscr{D}\left(g_{n}(t)\right) d t \rightarrow 0$. To this end, we recall that

$$
\frac{1}{2} \mathscr{D}\left(g_{n}\right)=-\left\langle\mathscr{C}\left(g_{n}\right), g_{n}\right\rangle_{\mathcal{L}^{2}}=-\left\langle\mathscr{C}^{+}\left(g_{n}\right), g_{n}\right\rangle_{\mathcal{L}^{2}}-\left\langle\mathscr{C}^{-}\left(g_{n}\right), g_{n}\right\rangle_{\mathcal{L}^{2}}
$$

and study each term separately. Using (7.12), we directly obtain that

$$
\int_{0}^{T}\left\langle\mathscr{C}^{+}\left(g_{n}\right), g_{n}\right\rangle_{\mathcal{L}^{2}} d t \rightarrow 0
$$

and it only remains to prove $\int_{0}^{T}\left\langle\mathscr{C}^{-}\left(g_{n}\right), g_{n}\right\rangle_{\mathcal{L}^{2}} d t \rightarrow 0$. We have

$$
\begin{aligned}
-\left\langle\mathscr{C}^{-}\left(g_{n}(t)\right), g_{n}(t)\right\rangle_{\mathcal{L}^{2}} & =\left\langle\left(\int_{\mathbb{R}^{d}} k\left(x, v, v^{\prime}\right) d v^{\prime}\right) g_{n}(t), g_{n}(t)\right\rangle_{\mathcal{L}^{2}} \\
& =\int_{\mathbb{T}^{d} \times \mathbb{R}^{d}}\left(\int_{\mathbb{R}^{d}} k\left(x, v, v^{\prime}\right) d v^{\prime}\right) v_{n}(t, x, v) \frac{e^{V}}{\mathcal{M}} d s d v d x \\
& =A_{n}(t)+B_{n}(t),
\end{aligned}
$$

with, according to (7.14),

$$
\begin{aligned}
A_{n}(t)= & \int_{\mathbb{T}^{d} \times \mathbb{R}^{d}}\left(\int_{\mathbb{R}^{d}} k\left(x, v, v^{\prime}\right) d v^{\prime}\right) e^{-2 \int_{0}^{t} \int_{\mathbb{R}^{d}} k\left(\phi_{s-t}(x, v), v^{\prime}\right) d v^{\prime} d s} v_{0, n}\left(\phi_{-t}(x, v)\right) \frac{e^{V}}{\mathcal{M}} d v d x \\
B_{n}(t)= & \int_{\mathbb{T}^{d} \times \mathbb{R}^{d}}\left(\int_{\mathbb{R}^{d}} k\left(x, v, v^{\prime}\right) d v^{\prime}\right) \int_{0}^{t} 2 \mathscr{C}^{+}\left(g_{n}(s)\right)\left(\phi_{s-t}(x, v)\right) g_{n}\left(s, \phi_{s-t}(x, v)\right) \\
& \times e^{-2 \int_{s}^{t} \int_{\mathbb{R}^{d}} k\left(\phi_{\tau-t}(x, v), v^{\prime}\right) d v^{\prime} d \tau} d s \frac{e^{V}}{\mathcal{M}} d v d x .
\end{aligned}
$$


We first have

$$
\begin{aligned}
0 \leq \int_{0}^{T} A_{n}(t) d t & \leq \int_{0}^{T} \int_{\mathbb{T}^{d} \times \mathbb{R}^{d}}\left(\int_{\mathbb{R}^{d}} k\left(x, v, v^{\prime}\right) d v^{\prime}\right) v_{0, n}\left(\phi_{-t}(x, v)\right) \frac{e^{V}}{\mathcal{M}} d v d x d t \\
& \leq \int_{0}^{T} \int_{\mathbb{T}^{d} \times \mathbb{R}^{d}}\left(\int_{\mathbb{R}^{d}} k\left(\phi_{t}(x, v), v^{\prime}\right) d v^{\prime}\right) v_{0, n}(x, v) \frac{e^{V}}{\mathcal{M}} d v d x d t \\
& \leq \int_{\mathbb{T}^{d} \times \mathbb{R}^{d}}\left(\int_{0}^{T} \int_{\mathbb{R}^{d}} k\left(\phi_{t}(x, v), v^{\prime}\right) d v^{\prime} d t\right) v_{0, n}(x, v) \frac{e^{V}}{\mathcal{M}} d v d x
\end{aligned}
$$

According to (7.13) (together with the fact that $v_{0, n}$ is uniformly compactly supported), we have, as $n \rightarrow \infty$ the following convergence

$$
\begin{gathered}
\int_{\mathbb{T}^{d} \times \mathbb{R}^{d}}\left(\int_{0}^{T} \int_{\mathbb{R}^{d}} k\left(\phi_{t}(x, v), v^{\prime}\right) d v^{\prime} d t\right) v_{0, n}(x, v) \frac{e^{V}}{\mathcal{M}} d v d x \\
\rightarrow\left(\int_{0}^{T} \int_{\mathbb{R}^{d}} k\left(\phi_{t}\left(x_{0}, v_{0}\right), v^{\prime}\right) d v^{\prime} d t\right) \frac{e^{V\left(x_{0}\right)}}{\mathcal{M}\left(v_{0}\right)}
\end{gathered}
$$

As a consequence of (7.11), there is $N_{0}$ such that for $n \geq N_{0}$, we have

$$
\int_{0}^{T} A_{n}(t) d t \leq \frac{2}{3} \varepsilon
$$

We now want to study the term $\int_{0}^{T} B_{n}(t) d t$. For this, we define the following new weighted $L^{2}$ norm

$$
\|f\|_{\mathbb{L}^{2}}^{2}:=\int_{\mathbb{T}^{d} \times \mathbb{R}^{d}}|f|^{2} \varphi^{2} \frac{e^{V}}{\mathcal{M}} d v d x
$$

where $\varphi$ is the function given by Assumption A3'. We have the following $\mathbb{L}^{2}$ estimate for the Boltzmann equation (1.1).

Lemma 7.1 For any function $f_{0} \in \mathcal{L}^{2}$ with $\left\|f_{0}\right\|_{\mathbb{L}^{2}}<+\infty$, the solution $f(t)$ to the Boltzmann equation (1.1) with initial datum $f_{0}$ satisfies, for all $t \geq 0$,

$$
\|f(t)\|_{\mathbb{L}^{2}} \leq\left\|f_{0}\right\|_{\mathbb{L}^{2}} e^{\Gamma t}
$$

where

$$
\Gamma:=\left(\sup _{x \in \mathbb{T}^{d}} \int_{\mathbb{R}^{d} \times \mathbb{R}^{d}} k^{2}\left(x, v^{\prime}, v\right) \frac{\mathcal{M}\left(v^{\prime}\right)}{\mathcal{M}(v)}\left(\frac{\varphi(x, v)}{\varphi\left(x, v^{\prime}\right)}-1\right)^{2} d v d v^{\prime}\right)^{\frac{1}{2}}
$$

which is finite by A3'. 
Proof of Lemma 7.1 The proof follows from an energy estimate for (1.1). We first multiply (1.1) by $f \varphi^{2}(x, v) \frac{e^{V}}{\mathcal{M}}$. Recalling that $\varphi$ is a function of the hamiltonian, we can integrate and argue as in Lemma 4.1 to treat the terms coming from the collision operator and thus obtain:

$$
\begin{aligned}
\frac{1}{2} \frac{d}{d t} & \|f(t)\|_{\mathbb{L}^{2}}^{2}+0 \\
= & \int_{\mathbb{T}^{d} \times \mathbb{R}^{d}}\left(\int_{\mathbb{R}^{d}} k\left(x, v^{\prime}, v\right)\left(1-\frac{\varphi\left(x, v^{\prime}\right)}{\varphi(x, v)}\right) f\left(t, x, v^{\prime}\right) d v^{\prime}\right) f \varphi^{2}(x, v) \frac{e^{V}}{\mathcal{M}} d v d x \\
& +\langle\mathscr{C}(f \varphi), f \varphi\rangle_{\mathcal{L}^{2}} \\
= & \int_{\mathbb{T}^{d} \times \mathbb{R}^{d}}\left(\int_{\mathbb{R}^{d}} k\left(x, v^{\prime}, v\right)\left(1-\frac{\varphi\left(x, v^{\prime}\right)}{\varphi(x, v)}\right) f\left(t, x, v^{\prime}\right) d v^{\prime}\right) f \varphi^{2}(x, v) \frac{e^{V}}{\mathcal{M}} d v d x \\
& -\frac{1}{2} \mathscr{D}(f \varphi) \\
\leq & \left(\int_{\mathbb{T}^{d} \times \mathbb{R}^{d}}\left(\int_{\mathbb{R}^{d}} k\left(x, v^{\prime}, v\right)\left(1-\frac{\varphi\left(x, v^{\prime}\right)}{\varphi(x, v)}\right) f\left(t, x, v^{\prime}\right) d v^{\prime}\right)^{2} \varphi^{2}(x, v) \frac{e^{V}}{\mathcal{M}} d v d x\right)^{\frac{1}{2}} \\
& \times\|f(t)\|_{\mathbb{L}^{2} .}
\end{aligned}
$$

Above we have used that the dissipation term $\mathscr{D}(f \varphi)$ is non-negative. Using the Cauchy-Schwarz inequality, we have, for fixed $x \in \mathbb{T}^{d}$

$$
\begin{aligned}
& \left(\int_{\mathbb{R}^{d}} k\left(x, v^{\prime}, v\right)\left(1-\frac{\varphi\left(x, v^{\prime}\right)}{\varphi(x, v)}\right) f\left(x, v^{\prime}\right) d v^{\prime}\right)^{2} \\
& \leq \quad\left(\int_{\mathbb{R}^{d}} f^{2}\left(x, v^{\prime}\right) \frac{\varphi^{2}\left(x, v^{\prime}\right)}{\mathcal{M}\left(v^{\prime}\right)} d v^{\prime} d x\right) \\
& \quad \times\left(\int_{\mathbb{R}^{d}} k^{2}\left(x, v^{\prime}, v\right)\left(1-\frac{\varphi\left(x, v^{\prime}\right)}{\varphi(x, v)}\right)^{2} \frac{\mathcal{M}\left(v^{\prime}\right)}{\varphi^{2}\left(x, v^{\prime}\right)} d v^{\prime}\right),
\end{aligned}
$$

from which we deduce $\frac{1}{2} \frac{d}{d t}\|f(t)\|_{\mathbb{L}^{2}}^{2} \leq \Gamma\|f(t)\|_{\mathbb{L}^{2}}^{2}$. This implies (7.17), and concludes the proof of the lemma.

By construction of the sequence $\left(g_{0, n}\right)$, it is uniformly compactly supported and we hence observe that there exists $C_{0}>0$ such that for all $n \in \mathbb{N}$,

$$
\left\|g_{0, n}\right\|_{\mathbb{L}^{2}} \leq C_{0}
$$

We thus use Lemma 7.1 to infer that there exists $C_{T}>0$, such that for all $n \in \mathbb{N}$, for all $t \in[0, T]$,

$$
\left\|g_{n}(t)\right\|_{\mathbb{L}^{2}} \leq C_{T}
$$


To estimate $\int_{0}^{T} B_{n}(t) d t$, we write

$$
\begin{aligned}
0 & \leq B_{n}(t) \leq \int_{\mathbb{T}^{d} \times \mathbb{R}^{d}}\left(\int_{\mathbb{R}^{d}} k\left(x, v, v^{\prime}\right) d v^{\prime}\right) \\
& \times \int_{0}^{t} 2 \mathscr{C}^{+}\left(g_{n}(s)\right)\left(\phi_{s-t}(x, v)\right) g_{n}\left(s, \phi_{s-t}(x, v)\right) d s \frac{e^{V}}{\mathcal{M}} d v d x
\end{aligned}
$$

and use A3'. We notice that since $\varphi$ is a function of the hamiltonian, we have

$$
\int_{\mathbb{R}^{d}} k\left(x, v, v^{\prime}\right) d v^{\prime} \leq \varphi(x, v)=\varphi\left(\phi_{s-t}(x, v)\right) .
$$

Therefore, using the change of variables $\phi_{s-t}(x, v) \mapsto(x, v)$, the Fubini theorem, and the Cauchy-Schwarz inequality, we obtain

$$
0 \leq B_{n}(t) \leq C\left\|\int_{\mathbb{R}^{d}} k\left(x, v^{\prime}, v\right) g_{n}\left(s, x, v^{\prime}\right) d v^{\prime}\right\|_{L^{1}\left([0, t] ; \mathcal{L}^{2}\right)[0, t]} \sup _{[0}\left\|g_{n}\right\|_{\mathbb{L}^{2}}
$$

By (7.12) and (7.18), we deduce that $\int_{0}^{T} B_{n}(t) \rightarrow 0$ as $n \rightarrow+\infty$. This, combined with (7.15) and (7.16) implies that the function $g_{0, \varepsilon}:=g_{0, n}$ (with $n$ large enough) satisfies (7.10), which concludes the proof of $\left(b^{\prime}.\right) \Longrightarrow(a$.$) .$

\subsection{Rigidity with respect to exponential convergence of the Maxwellian}

We prove here that (c.) implies (a.) in Theorem 2.3.

Assume that (c.) holds. By (1) implies (2) in Theorem 2.1, $\omega$ satisfies the a.e.i.t. GCC. Therefore, by Theorem 5.1, this means that $P f_{0}$ is of the form defined in (5.29). We use these notations again.

Recall by Lemma 5.2 that given an equivalence class $\left[\Omega_{j}\right]$ for $\sim$, denoting as usual $U_{j}=\bigcup_{\Omega^{\prime} \in\left[\Omega_{j}\right]} \Omega^{\prime}$, we have for all $t \geq 0$,

$$
\int_{U_{j}} f(t) d v d x=\int_{U_{j}} f_{0} d v d x
$$

where $f(t)$ is the solution of (1.1) with initial condition $f_{0}$.

Thus, the linear Boltzmann equation (1.1) is well-posed in the space

$$
\mathcal{L}_{00}^{2}:=\left\{f \in \mathcal{L}^{2}, \forall j \in J, \int_{U_{j}} f d v d x=0\right\}
$$

and we can use Lemma 11.1 for solutions in $\mathcal{L}_{00}^{2}$, which yields that the exponential convergence property is equivalent to 
(c'.) There exists $T>0$ and $K>0$ such that for all $f_{0} \in \mathcal{L}_{00}^{2}$, the associated solution $f$ to (1.1) satisfies

$$
K \int_{0}^{T} \mathscr{D}(f(t)) d t \geq\left\|f_{0}\right\|_{\mathcal{L}^{2}}^{2}
$$

We can then make the same proof as $\left(b^{\prime}.\right) \Longrightarrow(a$.) in Theorem 2.3 in order to infer that if $C^{-}(\infty)=0$, then $\left(c^{\prime}\right)$ does not hold. We keep the notations of that proof. The only thing to check is that $g_{0, n}$ defined there belongs to $\mathcal{L}_{00}^{2}$ for $n$ large enough. Let $j \in J$ such that $\left(x_{0}, v_{0}\right) \in U_{j}$. Then for $n$ large enough, supp $g_{0, n} \subset U_{j}$. Thus for all $i \neq j$, we have $\int_{U_{i}} g_{0, n} d v d x=0$ and

$$
\int_{U_{j}} g_{0, n} d v d x=\int_{\mathbb{T}^{d} \times \mathbb{R}^{d}} g_{0, n} d v d x=0,
$$

by definition of $g_{0, n}$. Thus $g_{0, n} \in \mathcal{L}_{00}^{2}$ for $n$ large enough, which concludes the proof of (c.) implies (a.) in Theorem 2.3.

\section{Remarks on Lower Bounds for Convergence when $C^{-}(\infty)=0$}

In the situation where $\omega$ satisfies a.e.i.t. GCC but $C^{-}(\infty)=0$, we know by Theorem 5.1 that for all data in $\mathcal{L}^{2}$ there is convergence to some $P f_{0}$ (defined in (5.29)). It is natural to wonder if there is a uniform decay rate for smoother data (in an appropriately defined way). If so, then the question of the convergence rate one can obtain becomes particularly interesting.

Let us provide here some a priori results in this direction. The following is nothing but a rephrasing in a general framework of a result of Bernard and Salvarani [4]. Note that they consider in their work free transport $(V=0)$ and velocities on the sphere $\mathbb{S}^{d-1}$, but one can readily check that their methods are relevant for (1.1). In their computations, one should add the weight $e^{V} / \mathcal{M}$ in the integrals.

Theorem 8.1 Denote $\left.\tau(x, v):=\inf \left\{t \geq 0, \phi_{-t}(x, v) \in \omega\right)\right\}$. Assume that there is a function of time $\varphi(t)$ such that

$$
\operatorname{Leb}\left\{(x, v) \in \mathbb{T}^{d} \times \mathbb{R}^{d}, \tau(x, v)>t\right\} \gtrsim \varphi(t) .
$$

Then, there exists a non-negative initial datum $f_{0}$ of $C^{\infty}$ class and $C>0$ such that for any $t \geq 0$, denoting by $f(t)$ the solution of the linear Boltzmann equation (1.1) with initial datum $f_{0}$, we have

$$
\left\|f(t)-P f_{0}\right\|_{\mathcal{L}^{2}} \geq C \varphi(t)
$$

where $P f_{0}$ is defined in (5.29).

In particular, we obtain 
Corollary 8.1 Assume that $V=0$ and that $\overline{p_{x}(\omega)} \neq \mathbb{T}^{d}$, where $p_{x}$ denotes the projection on the space of positions. Then there exists a non-negative initial datum $f_{0}$ of $C^{\infty}$ class and $C>0$ such that for any $t \geq 0$, denoting by $f(t)$ the solution of the linear Boltzmann equation with initial datum $f_{0}$, we have

$$
\left\|f(t)-P f_{0}\right\|_{\mathcal{L}^{2}} \geq \frac{C}{(1+t)^{d / 2}},
$$

where $P f_{0}$ is defined in (5.29).

Proof of Corollary 8.1 Take $x_{0} \in \mathbb{T}^{d} \backslash \overline{p_{x}(\omega)}$. Let $\delta:=\operatorname{dist}\left(x_{0}, \overline{p_{x}(\omega)}\right)$. Consider $U:=B\left(x_{0}, \delta / 2\right) \times B(0,1)$; here $\tau(x, v):=\inf \left\{t \geq 0, x-t v \in p_{x}(\omega)\right\}$. Then the crucial point is the straightforward lower bound

$$
\operatorname{Leb}\left\{(x, v) \in B\left(x_{0}, \delta / 2\right) \times B(0,1), \tau(x, v)>t\right\} \gtrsim \frac{1}{(1+t)^{d / 2}}
$$

and we can thus apply Theorem 8.1 .

Combining with Bernard-Salvarani's theorem which concerns the case with trapped trajectories [4], that we recall below, one may deduce that the "worst" lower bound in the free transport case is due to trapped trajectories, and not to low velocities.

Theorem 8.2 (Bernard-Salvarani [4]). Let $k$ a collision kernel belonging to the class E3" and $V=0$. Assume that there is $(x, v) \in \mathbb{T}^{d} \times \mathbb{R}^{d}$ such that for all $t \in \mathbb{R}^{+}$, $x+t v \notin \omega_{x}$. Then there exists a non-negative initial datum $f_{0}$ of $C^{\infty}$ class and $C>0$ such that for any $t \geq 0$, denoting by $f(t)$ the solution of the linear Boltzmann equation with initial datum $f_{0}$, we have

$$
\left\|f(t)-\left(\int_{\mathbb{T}^{d} \times \mathbb{R}^{d}} f_{0} d v d x\right) \mathcal{M}(v)\right\|_{\mathcal{L}^{2}} \geq C / \sqrt{1+t} .
$$

It is natural to conjecture that in this case, the bound in $1 / \sqrt{t}$ is optimal (this is supported by numerical evidence, as shown by De Vuyst and Salvarani [14]).

\section{The Case of a General Compact Riemannian Manifold}

In this section, we show how the above results adapt to a general Riemannian setting. More precisely, we explain how they apply to the linear Boltzmann equation written on the phase space $T^{*} M$ (it could be written on $T M$ equivalently) for a compact Riemannian manifold $M$, of which $\mathbb{T}^{d}$ is a (very) particular case.

Let $(M, g)$ be a smooth compact connected $d$-dimensional Riemannian manifold (without boundary). In local coordinates, the metric $g$ is a symmetric positive definite matrix such that for all $x \in M$ and $u, w \in T_{x} M$, we have

$$
(u, w)_{g(x)}=g_{i, j}(x) u^{i} w^{j},
$$


where the Einstein summation notations are used. This provides a canonical identification between the tangent bundle $T M$ and the cotangent bundle $T^{*} M$ via the following formula. For any covector $\eta \in T_{x}^{*} M$ there exists a unique vector $u \in T_{x} M$ satisfying

$$
\langle\eta, w\rangle_{T_{x}^{*} M, T_{x} M}=(u, w)_{g(x)}, \quad \text { for all } w \in T_{x} M .
$$

In local coordinates, we have

$$
\eta_{i}=g_{i, j}(x) u^{j}
$$

We can define an inner product on $T_{x}^{*} M$ using the above identification, denoted by $(\cdot, \cdot)_{g^{-1}(x)}$. In local coordinates, we have

$$
(\eta, \xi)_{g^{-1}(x)}=g^{i, j}(x) \eta_{i} \xi_{j}, \quad \text { where } g^{i, j}(x)=\left(g(x)^{-1}\right)^{i, j}
$$

For all $x \in M$ and all $\eta \in T_{x}^{*} M$, we denote by $|\eta|_{x}=(\eta, \eta)_{g^{-1}(x)}^{\frac{1}{2}}$ the associated norm. Let $d \operatorname{Vol}(x)$ be the canonical Riemannian measure on $M$. In local charts this reads

$$
d \operatorname{Vol}(x)=\sqrt{|\operatorname{det}(g(x))|} d x_{1} \cdots d x_{d} .
$$

Up to a renormalization factor, we may assume that $M$ has unit volume, i.e. $\operatorname{Vol}(M)=1$.

The cotangent bundle $T^{*} M$ is canonically endowed with a symplectic 2-form $\omega$ (in local charts, $\omega=\sum_{j=1}^{d} d x_{j} \wedge d \xi_{j}$ ). Let $\omega^{d}$ be the canonical symplectic volume form on $T^{*} M$ and by a slight abuse of notation $d \omega^{d}$ the associated normalized measure on $T^{*} M$ (see for instance [29, p. 274]). In local coordinates, we have

$$
d \omega^{d}=d \xi_{1} \cdots d \xi_{d} d x_{1} \cdots d x_{d}
$$

The canonical projection $\pi: T^{*} M \rightarrow M$ is measurable from $\left(T^{*} M, d \omega^{d}\right)$ to $\left(M, d\right.$ Vol). For $f \in L^{1}\left(T^{*} M, d \omega^{d}\right)$, we define $\pi_{*} f \in L^{1}(M, d$ Vol $)$ by

$\int_{M} \varphi(x)\left(\pi_{*} f\right)(x) d \operatorname{Vol}(x)=\int_{T^{*} M} \varphi \circ \pi(x, \xi) f(x, \xi) d \omega^{d}(x, \xi), \quad$ for all $\varphi \in C^{0}(M)$.

In local charts, we have

$$
\left(\pi_{*} f\right)(x)=\frac{1}{\sqrt{\operatorname{det}(g(x))}} \int_{\mathbb{R}^{d}} f(x, \xi) d \xi_{1} \cdots d \xi_{d} .
$$

Note also that we have the following desintegration formula

$$
\int_{T^{*} M} f(x, \xi) d \omega^{d}(x, \xi)=\int_{M} d \operatorname{Vol}(x) \int_{T_{x}^{*} M} f(x, \xi) d m_{x}(\xi),
$$


where the measure $d m_{x}$ on $T_{x}^{*} M$ is given in local charts by

$$
d m_{x}=\frac{1}{\sqrt{\operatorname{det}(g(x))}} d \xi_{1} \cdots d \xi_{d} .
$$

Let $V \in W^{2, \infty}(M)$, normalized so that $\int_{M} e^{-V(x)} d \operatorname{Vol}(x)=1$, and define on $T^{*} M$ the hamiltonian

$$
H(x, \xi)=\frac{1}{2}|\xi|_{x}^{2}+V(x), \quad x \in M, \quad \xi \in T_{x}^{*} M .
$$

We define the associated Hamilton vector field $X_{H}$, given in local coordinates by

$$
X_{H}=\nabla_{\xi} H \cdot \nabla_{x}-\nabla_{x} H \cdot \nabla_{\xi} .
$$

Using the 2-form $\omega$, we can also define the Poisson bracket $\{\cdot, \cdot\}$, see again [29, p. 271]. We have $X_{H} f=\{H, f\}$.

We denote by $\Lambda=\left\{\left(x, \xi, \xi^{\prime}\right), x \in M,\left(\xi, \xi^{\prime}\right) \in T_{x}^{*} M \times T_{x}^{*} M\right\}$ the vector bundle over $M$ whose fiber above $x$ is $T_{x}^{*} M \times T_{x}^{*} M$.

With these notations, the Boltzmann equation on $T^{*} M$ can be written as follows, for $(t, x, \xi) \in \mathbb{R} \times T^{*} M$,

$$
\begin{array}{rl}
\partial_{t} & f(t, x, \xi)+X_{H} f(t, x, \xi) \\
\quad=\int_{T_{x}^{*} M}\left[k\left(x, \xi^{\prime}, \xi\right) f\left(t, x, \xi^{\prime}\right)-k\left(x, \xi, \xi^{\prime}\right) f(t, x, \xi)\right] d m_{x}\left(\xi^{\prime}\right) .
\end{array}
$$

We recover the key properties of the usual linear Boltzmann collision operator on flat spaces. We have, for all $x \in M$,

$$
\int_{T_{x}^{*} M} \int_{T_{x}^{*} M}\left[k\left(x, \xi^{\prime}, \xi\right) f\left(x, \xi^{\prime}\right)-k\left(x, \xi, \xi^{\prime}\right) f(x, \xi)\right] d m_{x}\left(\xi^{\prime}\right) d m_{x}(\xi)=0 .
$$

Besides,

$$
\int_{T^{*} M}\left(X_{H} f\right)(x, \xi) d \omega^{d}(x, \xi)=0,
$$

since $\left(X_{H} f\right)(x, \xi) d \omega^{d}$ is an exact form (since $X_{H}$ is hamiltonian). As a consequence, the mass is conserved: any solution $f$ of (9.1) satisfies

$$
\text { for all } t \geq 0, \quad \frac{d}{d t} \int_{T^{*} M} f(t, x, \xi) d \omega^{d}(x, \xi)=0 .
$$

Consider now the (generalized) Maxwellian distribution:

$$
\mathcal{M}(x, \xi):=\frac{1}{(2 \pi)^{d / 2}} e^{-\frac{|\xi|_{x}^{2}}{2}}
$$


Note that for all $x \in M, \int_{T_{x}^{*} M} \frac{1}{(2 \pi)^{d / 2}} e^{-\frac{|\xi|_{x}^{2}}{2}} d m_{x}(\xi)=1$. As usual, we make the following assumptions on the collision kernel $k$.

A1. The collision kernel $k \in C^{0}(\Lambda)$, is nonnegative.

A2. We assume that the Maxwellian cancels the collision operator, that is:

$$
\begin{aligned}
& \int_{T_{x}^{*} M}\left[k\left(x, \xi^{\prime}, \xi\right) \mathcal{M}\left(x, \xi^{\prime}\right)-k\left(x, \xi, \xi^{\prime}\right) \mathcal{M}(x, \xi)\right] d m_{x}\left(\xi^{\prime}\right)=0, \\
& \quad \text { for all }(x, \xi) \in T^{*} M .
\end{aligned}
$$

A3. Assume that

$$
x \mapsto \int_{T_{x}^{*} M \times T_{x}^{*} M} k^{2}\left(x, \xi^{\prime}, \xi\right) \frac{\mathcal{M}\left(x, \xi^{\prime}\right)}{\mathcal{M}(x, \xi)} d m_{x}\left(\xi^{\prime}\right) d m_{x}(\xi) \in L^{\infty}(M)
$$

We can define the characteristics in this Riemannian setting as follows.

Definition 9.1 The hamiltonian flow $\left(\phi_{t}\right)_{t \in \mathbb{R}}$ associated to $H$ is the one parameter group of diffeomorphisms on $T^{*} M$ defined, for $(x, \xi) \in T^{*} M$, by $s \mapsto \phi_{s}(x, \xi) \in$ $T^{*} M$, where

$$
\frac{d}{d s} \phi_{s}(x, \xi)=X_{H}\left(\phi_{s}(x, \xi)\right), \quad \phi_{0}(x, \xi)=(x, \xi) \in T^{*} M
$$

The characteristic curve stemming from $(x, \xi) \in T^{*} M$ is the curve $\left\{\phi_{t}(x, \xi), t \in \mathbb{R}^{+}\right\}$.

Note also for any function $h$ defined on $\mathbb{R}, h \circ H$ is preserved along these integral curves, as

$$
\left.\left(\frac{d}{d s} h \circ H \circ \phi_{s}\right)\right|_{s=s_{0}}=X_{H}(h \circ H)\left(\phi_{s_{0}}\right)=\{H, h \circ H\}\left(\phi_{s_{0}}\right)=0 .
$$

In particular, this holds for the function $\frac{e^{V}}{\mathcal{M}}=\frac{1}{(2 \pi)^{d / 2}} e^{H}$.

With this definition of characteristics, we can then properly define

- the set $\omega$ where collisions are effective, as in Definition 2.1,

- $C^{-}(\infty)$, as in Definition 2.4,

- a.e.i.t. GCC, as in Definition 2.5,

- the Unique Continuation Property, as in Definition 2.11,

- the generalized Unique Continuation Property, as in Definition 5.1,

- the equivalence relations $\sim$ and $\approx$, as in Definitions 3.1 and 2.9,

- the sets $U_{j}$ as in (5.25).

Note that in this Riemannian setting, the classes of collision operators E1, E2, E3 still make sense, up to some obvious adaptations.

Let us now introduce the relevant weighted Lebesgue spaces. 
Definition 9.2 (Weighted $L^{p}$ spaces). We define the Banach spaces $\mathcal{L}^{2}$ and $\mathcal{L}^{\infty}$ by

$$
\begin{aligned}
& \mathcal{L}^{2}\left(T^{*} M\right):=\left\{f \in L_{l o c}^{2}\left(T^{*} M\right), \int_{T^{*} M}|f|^{2} \frac{e^{V}}{\mathcal{M}} d \omega^{d}<+\infty\right\}, \\
&\|f\|_{\mathcal{L}^{2}}=\left(\int_{T^{*} M}|f|^{2} \frac{e^{V}}{\mathcal{M}} d \omega^{d}\right)^{1 / 2} \cdot \\
& \mathcal{L}^{\infty}\left(T^{*} M\right):=\left\{f \in L_{l o c}^{1}\left(T^{*} M\right), \sup _{T^{*} M}|f| \frac{e^{V}}{\mathcal{M}}<+\infty\right\}, \quad\|f\|_{\mathcal{L}^{\infty}}=\sup _{T^{*} M}|f| \frac{e^{V}}{\mathcal{M}}
\end{aligned}
$$

The space $\mathcal{L}^{2}$ is a Hilbert space endowed with the inner product

$$
\langle f, g\rangle_{\mathcal{L}^{2}}:=\int_{T^{*} M} f g \frac{e^{V}}{\mathcal{M}} d \omega^{d} .
$$

As in the case of the torus, we have the following well-posedness result for the Boltzmann equation (9.1).

Proposition 9.1 (Well-posedness of the linear Boltzmann equation). Assume that $f_{0} \in \mathcal{L}^{2}$. Then there exists a unique $f \in C^{0}\left(\mathbb{R} ; \mathcal{L}^{2}\right)$ solution of (9.1) satisfying $\left.f\right|_{t=0}=f_{0}$, and we have

$$
\text { for all } t \geq 0, \quad \frac{d}{d t}\|f(t)\|_{\mathcal{L}^{2}}^{2}=-\mathscr{D}(f(t)),
$$

where

$$
\begin{aligned}
& \mathscr{D}(f)=\frac{1}{2} \int_{M} e^{V(x)} \int_{T_{x}^{*} M} \int_{T_{x}^{*} M}\left(\frac{k\left(x, \xi^{\prime}, \xi\right)}{\mathcal{M}(x, \xi)}+\frac{k\left(x, \xi, \xi^{\prime}\right)}{\mathcal{M}\left(x, \xi^{\prime}\right)}\right) \\
& \times \mathcal{M}(x, \xi) \mathcal{M}\left(x, \xi^{\prime}\right)\left(\frac{f(x, \xi)}{\mathcal{M}(x, \xi)}-\frac{f\left(x, \xi^{\prime}\right)}{\left.\mathcal{M}\left(x, \xi^{\prime}\right)\right)}\right)^{2} d m_{x}(\xi) d m_{x}\left(\xi^{\prime}\right) d \operatorname{Vol}(x) .
\end{aligned}
$$

If moreover $f_{0} \geq 0$ a.e., then for all $t \in \mathbb{R}$ we have $f(t, \cdot, \cdot) \geq 0$ a.e. (Maximum principle).

More generally, all results of Section 4 (up to obvious adaptations) are still relevant.

The crucial point we have to check now concerns velocity averaging lemmas for kinetic transport equations on a Riemannian manifold.

Lemma 9.1 Let $H$ be defined as above, and $X_{H}$ the associated vector field. Let $T>0$ and $\Psi \in C_{c}^{\infty}\left(T^{*} M\right)$. There exists $C>0$ a constant such that the following holds. For any $f, h \in L^{2}\left((0, T) \times T^{*} M\right)$ satisfying

$$
\partial_{t} f+X_{H} f=h,
$$

we have

$$
\left\|\pi_{*}(f \Psi)\right\|_{H^{1 / 4}((0, T) \times M)} \leq C\left(\left\|\left.f\right|_{t=0}\right\|_{L^{2}\left((0, T) \times T^{*} M\right)}+\|h\|_{L^{2}\left((0, T) \times T^{*} M\right)}\right) .
$$


i.e.

$$
\left\|\int_{T_{x}^{*} M} f \Psi d m_{x}\right\|_{H^{1 / 4}((0, T) \times M)} \leq C\left(\left\|\left.f\right|_{t=0}\right\|_{L^{2}\left((0, T) \times T^{*} M\right)}+\|h\|_{L^{2}\left((0, T) \times T^{*} M\right)}\right) .
$$

Remark 9.1 Assuming that $V$ is smooth enough, we may obtain the optimal Sobolev regularity $H^{1 / 2}$ (instead of $H^{1 / 4}$ ), see Remark 12.1 .

Proof of Lemma 9.1 In local charts, we have

$$
\pi_{*}(f \Psi)(x, \xi)=\int_{\mathbb{R}^{d}} f(x, \xi) \Psi(x, \xi) \frac{1}{\sqrt{\operatorname{det}(g(x))}} d \xi
$$

and $f$ satisfies the kinetic equation

$$
\partial_{t} f+g^{i, j}(x) \xi_{j} \partial_{x_{i}} f-\left(\frac{1}{2} \partial_{x_{i}} g^{j, k}(x) \xi_{j} \xi_{k}+\partial_{x_{i}} V(x)\right) \partial_{\xi_{i}} f=h
$$

We use the change of variables $\bar{f}\left(t, x, v^{i}\right)=f\left(t, x, g_{i, j} v^{j}\right)$ (we define as well $\bar{h}$ and $\bar{\Psi})$, which satisfies the equation

$$
\partial_{t} \bar{f}+v^{i} \partial_{x_{i}} \bar{f}-\left(\Gamma_{j, k}^{i}(x) v^{j} v^{k}+\partial_{x_{i}} V(x)\right) \partial_{v_{i}} \bar{f}=\bar{h},
$$

where $\Gamma_{j, k}^{i}(x)=\frac{1}{2} g^{i, \ell}(x)\left(\partial_{x_{j}} g_{k, \ell}(x)+\partial_{x_{k}} g_{j, \ell}(x)-\partial_{x_{\ell}} g_{j, k}(x)\right)$ are the Christoffel symbols. Using a classical averaging lemma (see (12.1) in Theorem 12.1 in Appendix 2 with $m=1$ and $s=0$ ), we deduce that

$$
\begin{aligned}
& \left\|\int_{\mathbb{R}^{d}} \bar{f} \bar{\Psi} \sqrt{\operatorname{det}(g(x))} d v\right\|_{H^{1 / 4}\left((0, T) \times \mathbb{R}^{d} \times \mathbb{R}^{d}\right)} \\
& \quad \leq C\left(\left\|\left.\bar{f}\right|_{t=0}\right\|_{L^{2}\left((0, T) \times \mathbb{R}^{d} \times \mathbb{R}^{d}\right)}+\|\bar{h}\|_{L^{2}\left((0, T) \times \mathbb{R}^{d} \times \mathbb{R}^{d}\right)}\right) .
\end{aligned}
$$

Going back to the original variables, we deduce that

$$
\begin{aligned}
& \left\|\int_{\mathbb{R}^{d}} f \Psi \frac{1}{\sqrt{\operatorname{det}(g(x))}} d v\right\|_{H^{1 / 4}\left((0, T) \times \mathbb{R}^{d} \times \mathbb{R}^{d}\right)} \\
& \quad \leq C\left(\left\|\left.f\right|_{t=0}\right\|_{L^{2}\left((0, T) \times \mathbb{R}^{d} \times \mathbb{R}^{d}\right)}+\|h\|_{L^{2}\left((0, T) \times \mathbb{R}^{d} \times \mathbb{R}^{d}\right)}\right),
\end{aligned}
$$

which proves our claim.

Equipped with this tool (more generally the analogues of all averaging lemmas of Appendix 2 can be obtained as well), we have the following analogue of the general convergence result of Theorem 5.1 (which includes Theorems 2.2 and 2.1). The same proof applies with only minor adaptations. Recall that the sets $\left(U_{j}\right)_{j \in J}$ are defined in $(5.25)$. 
Theorem 9.1 The following statements are equivalent.

(i.) The set $\omega$ satisfies the generalized Unique Continuation Property.

(ii.) The set $\omega$ satisfies the a.e.i.t. GCC.

(iii.) For all $f_{0} \in \mathcal{L}^{2}$, denote by $f(t)$ the unique solution to (9.1) with initial datum $f_{0}$. We have

$$
\left\|f(t)-P f_{0}\right\|_{\mathcal{L}^{2}} \rightarrow_{t \rightarrow+\infty} 0
$$

where

$$
P f_{0}(x, v)=\sum_{j \in J} \frac{1}{\left\|\mathbb{1}_{U_{j}} e^{-V} \mathcal{M}\right\|_{\mathcal{L}^{2}}}\left(\int_{U_{j}} f_{0} d \omega^{d}\right) f_{j}
$$

with $\left(U_{j}\right)_{j \in J}$ defined in (5.25) and $f_{j}=\frac{\mathbb{1}_{U_{j}} e^{-V} \mathcal{M}}{\left\|\mathbb{1}_{U_{j}} e^{-V} \mathcal{M}\right\|_{\mathcal{L}^{2}}}$.

(iv.) For all $f_{0} \in \mathcal{L}^{2}$, denote by $f(t)$ the unique solution to (9.1) with initial datum $f_{0}$. We have

$$
\left\|f(t)-P f_{0}\right\|_{\mathcal{L}^{2}} \rightarrow_{t \rightarrow+\infty} 0
$$

where $P f_{0}$ is a stationary solution of (9.1).

We obtain as well the analogue of Theorem 2.3. As in the torus case, we make the additional technical assumption:

A3'. Assume that there exists a continuous function $\varphi(x, \xi):=\Theta \circ H(x, \xi)$, with $\varphi \geq 1$, such that for all $(x, \xi) \in T^{*} M$, we have

$$
\int_{T_{x}^{*} M} k\left(x, \xi, \xi^{\prime}\right) d m_{x}\left(\xi^{\prime}\right) \leq \varphi(x, \xi)
$$

and

$$
\sup _{x \in M} \int_{T_{x}^{*} M \times T_{x}^{*} M} k^{2}\left(x, \xi^{\prime}, \xi\right) \frac{\mathcal{M}\left(x, \xi^{\prime}\right)}{\mathcal{M}(x, \xi)}\left(\frac{\varphi(x, \xi)}{\varphi\left(x, \xi^{\prime}\right)}-1\right)^{2} d m_{x}(\xi) d m_{x}\left(\xi^{\prime}\right)<+\infty
$$

Theorem 9.2 (Exponential convergence to equilibrium). Assume that the collision kernel satisfies A3'. The following statements are equivalent:

(a.) $C^{-}(\infty)>0$.

(b.) There exists $C>0, \gamma>0$ such that for any $f_{0} \in \mathcal{L}^{2}$, the unique solution to (9.1) with initial datum $f_{0}$ satisfies for all $t \geq 0$

$$
\left\|f(t)-\left(\int_{T^{*} M} f_{0} d \omega^{d}\right) e^{-V} \mathcal{M}\right\|_{\mathcal{L}^{2}} \leq C e^{-\gamma t}\left\|f_{0}-\left(\int_{T^{*} M} f_{0} d \omega^{d}\right) e^{-V} \mathcal{M}\right\|_{\mathcal{L}^{2}} .
$$


(c.) There exists $C>0, \gamma>0$ such that for any $f_{0} \in \mathcal{L}^{2}$, there exists $P f_{0}$ a stationary solution of (9.1) such that the unique solution to (9.1) with initial datum $f_{0}$ satisfies for all $t \geq 0$,

$$
\left\|f(t)-P f_{0}\right\|_{\mathcal{L}^{2}} \leq C e^{-\gamma t}\left\|f_{0}-P f_{0}\right\|_{\mathcal{L}^{2}}
$$

As a particular case of Theorem 9.1, we have the following corollary.

Corollary 9.1 Assume that $V=0$ and $\omega=T^{*} \omega_{x}$, where $\omega_{x}$ is a non-empty open subset of M. Suppose that the dynamics associated to $\left(\phi_{t}\right)_{t \geq 0}$ on

$$
S^{*} M=\left\{(x, \xi) \in T^{*} M, \frac{1}{2}|\xi|_{x}^{2}=1\right\}
$$

is ergodic. Then for all $f_{0} \in \mathcal{L}^{2}$, denoting by $f(t)$ the unique solution to (9.1) with initial datum $f_{0}$, we have

$$
\left\|f(t)-\left(\int_{T^{*} M} f_{0} d \omega^{d}\right) \mathcal{M}(v)\right\|_{\mathcal{L}^{2}} \rightarrow_{t \rightarrow+\infty} 0,
$$

Note that if the dynamics of $\left(\phi_{t}\right)_{t \geq 0}$ is ergodic on $S^{*} M$, then it is also ergodic on cosphere bundles of any positive radius (since for $V=0$, the flow is homogeneous of degree one).

Classical examples of Riemannian manifolds satisfying this dynamical assumption are given by compact Riemannian manifolds with negative curvature.

\section{The case of compact phase spaces}

Instead of studying the linear Boltzmann equation on the "whole" phase space $T^{*} M$, it is possible to consider this equation set on the "reduced" compact phase spaces

$$
\begin{gathered}
B_{H}^{*} M=\left\{(x, \xi) \in T^{*} M, H(x, \xi) \leq R\right\}, \quad S_{H}^{*} M=\left\{(x, \xi) \in T^{*} M, H(x, \xi)=R\right\} \\
\text { or } \mathcal{R}_{H}^{*} M=\left\{(x, \xi) \in T^{*} M, R \leq H(x, \xi) \leq R^{\prime}\right\}
\end{gathered}
$$

for $R^{\prime}>R>0$. Note that by continuity, the potential $V$ is always bounded from below (and above), so that $B_{H}^{*} M, S_{H}^{*} M$ and $\mathcal{R}_{H}^{*} M$ are indeed compact.

We focus now on the case of $S_{H}^{*} M$ (the other cases being handled similarly). We require that the collision kernel $k$ is defined on

$$
\Gamma=\left\{\left(x, \xi, \xi^{\prime}\right), x \in M,\left(\xi, \xi^{\prime}\right) \in T_{x}^{*} M \times T_{x}^{*} M, H(x, \xi)=R, H\left(x, \xi^{\prime}\right)=R\right\}
$$

and make the following assumptions

A1. The collision kernel $k \in C^{0}(\Gamma)$, is nonnegative. 
A2. We assume that the Maxwellian cancels the collision operator, that is:

$$
\begin{aligned}
& \int_{\xi^{\prime} \in T_{x}^{*} M, H\left(x, \xi^{\prime}\right)=R}\left[k\left(x, \xi^{\prime}, \xi\right) \mathcal{M}\left(x, \xi^{\prime}\right)-k\left(x, \xi, \xi^{\prime}\right) \mathcal{M}(x, \xi)\right] d m_{x}\left(\xi^{\prime}\right)=0 \\
& \text { for all }(x, \xi) \in S_{H}^{*} M .
\end{aligned}
$$

Note that in the compact framework, the former assumption $\mathbf{A 3}$ is useless.

Within these assumptions, the linear Boltzmann equation (1.1) is well-posed in appropriate weighted Lebesgue spaces based on $L^{2}\left(S_{H}^{*} M\right)$, in particular because the hamiltonian is preserved by the dynamics. The case of $S_{H}^{*} M$ is for instance relevant for the equations of radiative transfer or neutronics.

The analogues of Theorems 2.2, 2.3, 5.1 still hold in this framework, up to the appropriate modifications of the various geometric conditions. For the sake of conciseness, we do not write these results again. All proofs remain valid, with some simplifications, since the phase space is now compact. Note that the fact that $C^{-}(\infty)>0$ is equivalent to GCC in this compact case.

Acknowledgments We wish to thank Diogo Arsénio for several interesting and stimulating discussions related to this work.

\section{Appendix 1: A Stabilization Criterion}

We provide in this appendix a characterization of exponential decay for dissipative evolution equations. The following lemma is very classical and we reproduce it here for the convenience of the reader.

Lemma 11.1 Consider the evolution equation

$$
\left\{\begin{array}{r}
\partial_{t} f+L f=0, \\
f_{\mid t=0}=f_{0}
\end{array}\right.
$$

assumed to be:

- globally wellposed in some functional space $X$ in the sense that for any $f_{0} \in X$, there is a unique $f \in C_{t}^{0}(X)$ solution to (11.1),

- invariant by translation in time, in the sense that if $f \in C_{t}^{0}(X)$ is the solution of (11.1), then for all $t_{0} \geq 0, g(t):=f\left(t+t_{0}\right)$ is the unique solution of

$$
\left\{\begin{array}{l}
\partial_{t} g+L g=0 \\
g_{\mid t=0}=f_{\mid t=t_{0}}
\end{array}\right.
$$

Let $\mathscr{E}(f)$ and $\mathscr{D}(f)$ be two non-negative functionals defined for all $f \in X$, and such that if $f$ is a solution to (11.1),

$$
\text { for all } t \geq t^{\prime} \geq 0, \quad \mathscr{E}(f(t))-\mathscr{E}\left(f\left(t^{\prime}\right)\right)=-\int_{t^{\prime}}^{t} \mathscr{D}(f(s)) d s
$$


Then, the following two properties are equivalent:

(1) There exist $C, \gamma>0$ such that for all $f(0) \in X$, the associated solution $f$ to (11.1) satisfies

$$
\text { for all } t \geq 0, \quad \mathscr{E}(f(t)) \leq C e^{-\gamma t} \mathscr{E}(f(0))
$$

(2) There exists $T>0$ and $K>0$ such that for all $f(0) \in X$, the associated solution $f$ to (11.1) satisfies

$$
K \int_{0}^{T} \mathscr{D}(f(t)) d t \geq \mathscr{E}(f(0))
$$

For the sake of completeness, we provide a short proof of this lemma.

Proof of Lemma 11.1 (1) $\Rightarrow$ (2) Assume that (1) holds. Let $T_{0}>0$ such that $C e^{-\gamma T_{0}}=\frac{1}{2}$. Then, using (11.3) betwen 0 and $T_{0}$, we have:

$$
\mathscr{E}\left(f\left(T_{0}\right)\right)-\mathscr{E}(f(0))=-\int_{0}^{T_{0}} \mathscr{D}(f(t)) d t
$$

so that, by (11.4),

$$
\int_{0}^{T_{0}} \mathscr{D}(f(t)) d t \geq \mathscr{E}(f(0))-C e^{-\gamma T_{0}} \mathscr{E}(f(0))=\frac{1}{2} \mathscr{E}(f(0)),
$$

and we can therefore take $T=T_{0}$ and $C=2$ in (11.5).

(2) $\Rightarrow$ (1) Assume that (2) holds. Here (and only here), we need the property of invariance by time translations for (11.1). By (11.3) and (11.5), we have

$$
\mathscr{E}(f(T)) \leq\left(1-\frac{1}{K}\right) \mathscr{E}(f(0)) .
$$

Note that the assumption $\mathscr{E}(f) \geq 0$ implies in particular that $K \geq 1$. We may assume that $K>1$. Indeed, for $K=1$, we have $\mathscr{E}(f(t))=0$ for all $t \geq T$ so that for any $\gamma>0$, there exists $C>0$ such that (1) holds. By invariance by translation in time of (11.1), one likewise obtains

$$
\mathscr{E}(f(2 T)) \leq\left(1-\frac{1}{K}\right) \mathscr{E}(f(T))
$$

Thus, by a straightforward induction, for any $k \in \mathbb{N}$, we have the bound:

$$
\mathscr{E}(f(k T)) \leq\left(1-\frac{1}{K}\right)^{k} \mathscr{E}(f(0))
$$


Defining $\gamma_{0}:=\frac{-\log \left(1-\frac{1}{K}\right)}{T}>0$ and $C_{0}:=\left(1-\frac{1}{K}\right)^{-1}=e^{\gamma_{0} T}>0$, we can now check that

$$
\text { for all } t \geq 0, \quad \mathscr{E}(f(t)) \leq C_{0} e^{-\gamma_{0} t} \mathscr{E}(f(0))
$$

Indeed, let $t \geq 0$ and $k \in \mathbb{N}$ such that $t \in[k T,(k+1) T[$; since $\mathscr{E}(f(\cdot))$ is decreasing (see (11.3)), we have

$\mathscr{E}(f(t)) \leq \mathscr{E}(f(k T)) \leq\left(1-\frac{1}{K}\right)^{k} \mathscr{E}(f(0))=e^{-\gamma_{0} k T} \mathscr{E}(f(0)) \leq C_{0} e^{-\gamma_{0} t} \mathscr{E}(f(0))$

which concludes the proof.

\section{Appendix 2: Velocity Averaging Lemmas}

Velocity averaging lemmas play an important role in many proofs of this paper. In this appendix, we recall some classical results and also state the versions precisely adapted to our needs.

Kinetic transport equations are hyperbolic partial differential equations and as it can be seen from Duhamel's formula, there is propagation of potential singularities at initial time and/or from a source in the equations. Thus there is no hope that the solution of a kinetic equation becomes more regular than the initial condition.

It was nevertheless observed by Golse, Perthame and Sentis [24] that the averages in velocity of the solution of a kinetic transport equation enjoy extra regularity/compactness properties (see also the independent paper of Agoshkov [1]). We refer to the by now classical paper of Golse, Lions, Perthame, Sentis [23], DiPerna, Lions [18], DiPerna, Lions, Meyer [19], Bézard [8] for quantitative estimates of this compactness property in various settings of increasing complexity.

We also refer to the review paper of Jabin [30] and to the recent work of Arsénio and Saint-Raymond [2].

We start by recalling classical averaging lemmas in the whole space $\mathbb{R}^{d}$. There are also versions of these lemmas for $p \in(1, \infty)$, but we stick to the case $p=2$, which is sufficient for our needs.

Theorem 12.1 (Kinetic averaging lemma $[8,18,19,23]$ ). Let $s \in[0,1)$ and $m \in \mathbb{R}^{+}$. For any $T>0$ and any bounded open sets $\Omega_{x}, \Omega_{v} \subset \mathbb{R}^{d}$, there exists a constant $C>0$ such that for all $\Psi \in C_{c}^{\infty}\left(\mathbb{R}^{d}\right)$ supported in $\Omega_{v}$ and all $f, g \in L_{l o c}^{2}\left(\mathbb{R} \times \mathbb{R}^{d} \times \mathbb{R}^{d}\right)$ satisfying

$$
\partial_{t} f+v \cdot \nabla_{x} f=\left(1-\Delta_{t, x}\right)^{s / 2}\left(1-\Delta_{v}\right)^{m / 2} g,
$$

we have

$$
\left\|\rho_{\Psi}\right\|_{H^{\alpha}\left([0, T] \times \Omega_{x}\right)} \leq C\left(\|f\|_{L^{2}\left([0, T] \times \Omega_{x} \times \Omega_{v}\right)}+\|g\|_{L^{2}\left([0, T] \times \Omega_{x} \times \Omega_{v}\right)}\right),
$$


where $\rho_{\Psi}(t, x):=\int_{\mathbb{R}^{d}} f(t, x, v) \Psi(v) d v$ and $\alpha=\frac{(1-s)}{2(1+m)}$.

As a consequence, we obtain

Corollary 12.1 Let $T>0$ and $\left(f_{n}\right)_{n \in \mathbb{N}}$ and $\left(g_{n}\right)_{n \in \mathbb{N}}$ be two sequences of $L^{2}\left(0, T ; L_{\text {loc }}^{2}\left(\mathbb{R}^{d} \times \mathbb{R}^{d}\right)\right)$ such that the following holds

$$
\partial_{t} f_{n}+v \cdot \nabla_{x} f_{n}=\left(1-\Delta_{t, x}\right)^{s / 2}\left(1-\Delta_{v}\right)^{m / 2} g_{n},
$$

with $s \in[0,1), m \geq 0$. Assume that for any bounded open sets $\Omega_{x}, \Omega_{v} \subset \mathbb{R}^{d}$, there exists $C_{1}>0$, such that for all $n \in \mathbb{N}$,

$$
\left\|f_{n}\right\|_{L^{2}\left((0, T) \times \Omega_{x} \times \Omega_{v}\right)}+\left\|g_{n}\right\|_{L^{2}\left((0, T) \times \Omega_{x} \times \Omega_{v}\right)} \leq C_{1} .
$$

Then, for any $\Psi \in C_{c}^{\infty}\left(\mathbb{R}^{d}\right)$, the sequence $\left(\rho_{\Psi, n}\right)_{n \in \mathbb{N}}$ defined for $n \in \mathbb{N}$ by

$$
\rho_{\Psi, n}(t, x):=\int_{\mathbb{R}^{d}} f_{n}(t, x, v) \Psi(v) d v
$$

is relatively compact in $L^{2}\left(0, T ; L_{l o c}^{2}\left(\mathbb{R}^{d}\right)\right)$.

Remark 12.1 In the main part of the paper, we apply this averaging lemma to the Boltzmann equation (1.1) by writing it under the form

$$
\partial_{t} f+v \cdot \nabla_{x} f=\nabla_{x} V \cdot \nabla_{v} f+\int_{\mathbb{R}^{d}}\left[k\left(x, v^{\prime}, v\right) f\left(v^{\prime}\right)-k\left(x, v, v^{\prime}\right) f(v)\right] d v^{\prime} .
$$

To this end, we consider the case $s=0, m=1$ in Theorem 12.1. This implies that the averages in $v$ belong to the Sobolev space $H^{1 / 4}$.

Nevertheless, assuming that the potential $V$ is smooth enough, we can also use the approach of Gérard-Golse [22] or Berthelin-Junca [7] to obtain the optimal Sobolev space $H^{1 / 2}$ for these averages (which is not needed in this paper).

Remark 12.2 We also have a version of these lemmas for kinetic transport equations set in general Riemannian manifolds, see Lemma 9.1.

We now state the result as needed in the main part of this work. Assuming an extra uniform integrability, we can deduce some compactness on moments of $f$ without having to consider compactly supported test functions in $v$. This is the purpose of the next result, which is actually the version of averaging lemmas used most of the time in this work.

Corollary 12.2 Let $\Omega_{x}$ be a bounded open set of $\mathbb{R}^{d}, T>0$, and $\left(f_{n}\right)_{n \in \mathbb{N}},\left(g_{n}\right)_{n \in \mathbb{N}}$ be two sequences of $L^{2}\left(0, T ; L_{\text {loc }}^{2}\left(\Omega_{x} \times \mathbb{R}^{d}\right)\right)$ satisfying $\partial_{t} f_{n}+v \cdot \nabla_{x} f_{n}=\left(1-\Delta_{v}\right)^{m / 2} g_{n}$, for some $m \geq 0$. Suppose that there exists $V \in L^{\infty}$ such that for any bounded open set $\Omega_{v} \subset \mathbb{R}^{d}$, there exists $C_{0}>0$ such that, for any $n \in \mathbb{N}$,

$$
\begin{aligned}
& \text { for all } t \geq 0, \quad\left\|f_{n}\right\|_{\mathcal{L}^{2}\left(\Omega_{x} \times \mathbb{R}^{d}\right)}^{2}:=\int_{\Omega_{x}} \int_{\mathbb{R}^{d}}\left|f_{n}\right|^{2} \frac{e^{V(x)}}{\mathcal{M}(v)} d v d x \leq C_{0}, \\
& \qquad\left\|g_{n}\right\|_{L^{2}\left((0, T) \times \Omega_{x} \times \Omega_{v}\right)} \leq C_{0} .
\end{aligned}
$$


Assume moreover that there is $f \in L^{\infty}\left(0, T ; L^{2}\left(\Omega_{x} \times \mathbb{R}^{d}\right)\right)$ such that $f_{n} \rightarrow f$ weakly- in $^{\infty}\left(0, T ; \mathcal{L}^{2}\left(\Omega_{x} \times \mathbb{R}^{d}\right)\right)$. Consider $\rho_{n}(t, x):=\int_{\mathbb{R}^{d}} f_{n}(t, x, v) d v$. Then up to a subsequence, we have

$$
\rho_{n} \mathcal{M}(v) \rightarrow\left(\int_{\mathbb{R}^{d}} f d v\right) \mathcal{M}(v), \quad \text { strongly in } L^{2}\left(0, T ; \mathcal{L}^{2}\left(\Omega_{x} \times \mathbb{R}^{d}\right)\right),
$$

and for any continuous kernel $k(\cdot, \cdot, \cdot): \mathbb{R}^{d} \times \mathbb{R}^{d} \times \mathbb{R}^{d} \rightarrow \mathbb{R}$ satisfying $\mathbf{A 3}$, we have

$$
\begin{aligned}
& \int_{\mathbb{R}^{d}} k\left(x, v^{\prime}, v\right) f_{n}\left(t, x, v^{\prime}\right) d v^{\prime} \rightarrow \int_{\mathbb{R}^{d}} k\left(x, v^{\prime}, v\right) f\left(t, x, v^{\prime}\right) d v^{\prime}, \\
& \text { strongly in } L^{2}\left(0, T ; \mathcal{L}^{2}\left(\Omega_{x} \times \mathbb{R}^{d}\right)\right) .
\end{aligned}
$$

Moreover, the same result still holds if we replace $\Omega_{x}$ by $\mathbb{T}^{d}$.

Proof of Corollary 12.2 Note first that the result for $\mathbb{T}^{d}$ can be deduced by a finite covering of $\mathbb{T}^{d}$ by local charts of the form $\Omega_{x}$. Hence, it suffices to prove the result with $\Omega_{x}$. By Fatou's lemma, the function $f$ satisfies:

$$
\text { for all } t \geq 0, \quad\|f\|_{\mathcal{L}^{2}}^{2}=\int_{\Omega_{x}} \int_{\mathbb{R}^{d}}|f|^{2} \frac{e^{V(x)}}{\mathcal{M}(v)} d v d x \leq C_{0} .
$$

Since $\rho_{n}$ does not depend on $v$, proving (12.4) is equivalent to show that:

$$
\rho_{n} e^{V} \rightarrow \int_{\mathbb{R}^{d}} f d v e^{V}, \quad \text { strongly in } L^{2}\left((0, T) \times \Omega_{x}\right)
$$

Let $\Psi \in C_{c}^{\infty}(\mathbb{R})$ such that $\Psi=1$ in a neighborhood of 0 , and define $\Psi_{R}(v)=\Psi\left(\frac{|v|}{R}\right)$, $v \in \mathbb{R}^{d}$.

By Corollary 12.1, we can assume, up to a subsequence, that

$$
\rho_{\Psi_{R}, n}:=\int_{\mathbb{R}^{d}} f_{n} \Psi_{R} d v \rightarrow \int_{\mathbb{R}^{d}} f \Psi_{R} d v, \quad \text { strongly in } L^{2}\left((0, T) \times \Omega_{x}\right) .
$$

Let $\varepsilon>0$. We can write the decomposition:

$$
\begin{aligned}
& \rho_{n}-\int_{\mathbb{R}^{d}} f d v=A_{1}+A_{2}+A_{3}, \quad \text { with } \\
& \begin{array}{l}
A_{1}=\left(\rho_{\Psi_{R}, n}-\int_{\mathbb{R}^{d}} f \Psi_{R} d v\right), \quad A_{2}=\int_{\mathbb{R}^{d}} f_{n}\left(1-\Psi_{R}\right) d v, \\
A_{3}=-\int_{\mathbb{R}^{d}} f\left(1-\Psi_{R}\right) d v .
\end{array}
\end{aligned}
$$


By Cauchy-Schwarz inequality, using (12.3), we have for all $n \in \mathbb{N}$ and all $t \in(0, T)$,

$$
\begin{aligned}
& \left\|\int_{\mathbb{R}^{d}} f_{n}\left(1-\Psi_{R}\right) d v e^{V}\right\|_{L^{2}\left(\Omega_{x}\right)}^{2} \\
& \quad \leq \int\left(\int_{\mathbb{R}^{d}}\left|f_{n}\right|^{2} \frac{1}{\mathcal{M}(v)} d v\right)\left(\int_{\mathbb{R}^{d}}\left(1-\Psi_{R}\right)^{2} \mathcal{M}(v) d v\right) e^{2 V(x)} d x \\
& \quad \leq C_{0}\left\|e^{V}\right\|_{L^{\infty}\left(\Omega_{x}\right)}\left(\int_{\mathbb{R}^{d}}\left(1-\Psi_{R}\right)^{2} \mathcal{M}(v) d v\right) .
\end{aligned}
$$

As a consequence, there exists $R_{0}>0$ large enough such that for all $R \geq R_{0}$ and all $n \in \mathbb{N}$, we have

$$
\left\|e^{V} A_{2}\right\|_{L^{2}\left((0, T) \times \Omega_{x}\right)}^{2} \leq \frac{\varepsilon}{3}
$$

Likewise, we use (12.6) to get for all $n \in \mathbb{N}$,

$$
\left\|e^{V} A_{3}\right\|_{L^{2}\left((0, T) \times \Omega_{x}\right)}^{2} \leq \frac{\varepsilon}{3} .
$$

Using (12.8) ( $R$ is now fixed), there is $N \geq 0$ such that for any $n \geq N$,

$$
\left\|\left(\rho_{\Psi_{R}, n}-\int_{\mathbb{R}^{d}} f \Psi_{R} d v\right) e^{V}\right\|_{L^{2}\left((0, T) \times \Omega_{x}\right)} \leq \varepsilon / 3,
$$

from which we infer that

$$
\left\|\left(\rho_{n}-\int_{\mathbb{R}^{d}} f d v\right) e^{V}\right\|_{L^{2}\left((0, T) \times \Omega_{x}\right)} \leq \varepsilon
$$

and this concludes the proof of (12.4).

For the proof of (12.5), let us first assume for a while that $k$ is smooth (namely for all $x, k(x, \cdot, \cdot)$ belongs to the $C^{\infty}$ class). We first have to be careful about the integration in the velocity variable. The convergence in (12.5) results from the following two facts:

- For all $v \in \mathbb{R}^{d}$, we have the following convergence

$$
\begin{aligned}
& \int_{\mathbb{R}^{d}} k\left(x, v^{\prime}, v\right) f_{n}\left(t, x, v^{\prime}\right) d v^{\prime} \\
& \quad \rightarrow \int_{\mathbb{R}^{d}} k\left(x, v^{\prime}, v\right) f\left(t, x, v^{\prime}\right) d v^{\prime} \quad \text { strongly in } L^{2}\left(0, T ; L_{x}^{2}\left(\Omega_{x}\right)\right) .
\end{aligned}
$$

This follows from a truncation argument and Corollary 12.1, exactly as for $\rho_{n}$. Keeping the same notations, the only difference is that we have to study

$$
\left(\int_{\mathbb{R}^{d}}\left(1-\Psi_{R}\right)^{2} k^{2}\left(x, v^{\prime}, v\right) \mathcal{M}\left(v^{\prime}\right) d v^{\prime}\right),
$$


which, using A3, is small for $R$ large enough.

- By the Cauchy-Schwarz inequality and the bound (12.3), we have

$$
\begin{aligned}
& \int_{0}^{T} \int_{\Omega_{x}}\left(\int_{\mathbb{R}^{d}} k\left(x, v^{\prime}, v\right)\left(f_{n}-f\right)\left(t, x, v^{\prime}\right) d v^{\prime}\right)^{2} \frac{e^{V}}{\mathcal{M}(v)} d x d t \\
& \leq \int_{0}^{T}\left(\sup _{x \in \Omega_{x}} \int_{\mathbb{R}^{d}} k^{2}\left(x, v^{\prime}, v\right) \frac{\mathcal{M}\left(v^{\prime}\right)}{\mathcal{M}(v)} d v^{\prime}\right) \\
& \int_{\Omega_{x}}\left(\int_{\mathbb{R}^{d}} \frac{\left|f_{n}-f\right|^{2}\left(t, x, v^{\prime}\right)}{\mathcal{M}\left(v^{\prime}\right)} d v^{\prime}\right) e^{V} d x d t \\
& \leq C_{0} T \sup _{x \in \Omega_{x}} \int_{\mathbb{R}^{d}} k^{2}\left(x, v^{\prime}, v\right) \frac{\mathcal{M}\left(v^{\prime}\right)}{\mathcal{M}(v)} d v^{\prime},
\end{aligned}
$$

which is independent of $n$ and in $L^{1}(d v)$, since by $\mathbf{A} \mathbf{3}$, we have

$$
\sup _{x \in \Omega_{x}} \int_{\mathbb{R}^{d} \times \mathbb{R}^{d}} k^{2}\left(x, v^{\prime}, v\right) \frac{\mathcal{M}\left(v^{\prime}\right)}{\mathcal{M}(v)} d v^{\prime} d v<+\infty .
$$

Hence, by Lebesgue dominated convergence theorem, we deduce (12.5).

We now use an approximation argument to handle the general case, i.e. when $k$ is only assumed to be continuous. Consider $\left(\phi_{\delta}\right)_{\delta>0}$ a family of mollifiers in $C_{c}^{\infty}\left(\mathbb{R}^{d} \times\right.$ $\mathbb{R}^{d}$ ) for the measure $\mathcal{M}(v) \mathcal{M}\left(v^{\prime}\right) d v^{\prime} d v$. We set for all $x, v, v^{\prime}$

$$
\tilde{k}_{\delta}\left(x, v, v^{\prime}\right)=\left(\tilde{k}(x, \cdot, \cdot) \star \phi_{\delta}(\cdot, \cdot)\right)\left(v, v^{\prime}\right), \quad k_{\delta}\left(x, v, v^{\prime}\right)=\tilde{k}_{\delta}\left(x, v, v^{\prime}\right) \mathcal{M}\left(v^{\prime}\right) .
$$

We use the following classical properties of mollifiers:

- for all $x, k_{\delta}(x, \cdot, \cdot)$ is in the $C^{\infty}$ class;

- we have for all $\delta>0$

$$
\sup _{x \in \Omega_{x}}\left\|\tilde{k}-k_{\delta}\right\|_{L^{2}\left(\mathcal{M}(v) \mathcal{M}\left(v^{\prime}\right) d v^{\prime} d v\right)} \rightarrow_{\delta \rightarrow 0} 0 .
$$

Let $\varepsilon>0$. We write the decomposition

$$
\left\|\int_{\mathbb{R}^{d}} k\left(x, v^{\prime}, v\right) f_{n}\left(t, x, v^{\prime}\right) d v^{\prime}-\int_{\mathbb{R}^{d}} k\left(x, v^{\prime}, v\right) f\left(t, x, v^{\prime}\right) d v^{\prime}\right\|_{\mathcal{L}^{2}}^{2} \leq 2 A_{1}+2 A_{2},
$$

with

$$
\begin{aligned}
& A_{1}=\left\|\int_{\mathbb{R}^{d}} k_{\delta}\left(x, v^{\prime}, v\right) f_{n}\left(t, x, v^{\prime}\right) d v^{\prime}-\int_{\mathbb{R}^{d}} k_{\delta}\left(x, v^{\prime}, v\right) f\left(t, x, v^{\prime}\right) d v^{\prime}\right\|_{\mathcal{L}^{2}}^{2}, \\
& A_{2}=\left\|\int_{\mathbb{R}^{d}}\left(k-k_{\delta}\right)\left(x, v^{\prime}, v\right)\left(f_{n}-f\right)\left(t, x, v^{\prime}\right) d v^{\prime}\right\|_{\mathcal{L}^{2}}^{2} .
\end{aligned}
$$


We estimate $A_{2}$ as follows, using (12.10)

$$
\begin{aligned}
A_{2}= & \int_{\mathbb{R}^{d}} \mathcal{M}(v) \int_{\Omega_{x}}\left(\int_{\mathbb{R}^{d}}\left(\tilde{k}-\tilde{k}_{\delta}\right)\left(x, v^{\prime}, v\right)\left(f_{n}-f\right)\left(t, x, v^{\prime}\right) d v^{\prime}\right)^{2} e^{V} d x d v \\
\leq & \sup _{x \in \Omega_{x}} \int_{\mathbb{R}^{d} \times \mathbb{R}^{d}}\left|\tilde{k}-\tilde{k}_{\delta}\right|^{2}\left(x, v^{\prime}, v\right) \mathcal{M}\left(v^{\prime}\right) \mathcal{M}(v) d v^{\prime} d v \\
& \times\left(\int_{\Omega_{x}} \int_{\mathbb{R}^{d}} \frac{\left|f_{n}-f\right|^{2}\left(t, x, v^{\prime}\right)}{\mathcal{M}\left(v^{\prime}\right)} e^{V} d v^{\prime} d x\right) \\
\leq & 4 C_{0}^{2} \sup _{x \in \Omega_{x}} \int_{\mathbb{R}^{d} \times \mathbb{R}^{d}}\left|\tilde{k}-\tilde{k}_{\delta}\right|^{2}\left(x, v^{\prime}, v\right) \mathcal{M}\left(v^{\prime}\right) \mathcal{M}(v) d v^{\prime} d v .
\end{aligned}
$$

Using (12.10), we fix $\delta>0$ small enough so that for all $n \in \mathbb{N}$,

$$
A_{2} \leq(\varepsilon /(4 T))^{1 / 2} \text {. }
$$

and thus for all $n \in \mathbb{N}$, we have

$$
\left\|A_{2}\right\|_{L^{2}(0, T)}^{2} \leq \varepsilon / 4 .
$$

For $A_{1}$, we use the above analysis in the smooth case to deduce that we can take $N$ large enough to get for all $n \geq N$,

$$
\left\|A_{1}\right\|_{L^{2}(0, T)}^{2} \leq \varepsilon / 4 .
$$

Finally, we have proven that for any $\varepsilon>0$, there is $N$ such that for all $n \geq N$,

$$
\left\|\int_{\mathbb{R}^{d}} k\left(x, v^{\prime}, v\right) f_{n}\left(t, x, v^{\prime}\right) d v^{\prime}-\int_{\mathbb{R}^{d}} k\left(x, v^{\prime}, v\right) f\left(t, x, v^{\prime}\right) d v^{\prime}\right\|_{L^{2}\left(0, T ; \mathcal{L}^{2}\right)}^{2} \leq \varepsilon,
$$

which concludes the proof of the convergence.

\section{Appendix 3: Reformulation of Some Geometric Properties}

In this appendix, we first provide a proof of Lemma 3.1, giving the link between the two equivalence relations $\approx$ and $\sim$. Then, we explore related geometric conditions with additional connectedness assumptions, that are sufficient for having a single equivalence class for $\approx$ and $\sim$.

\section{Appendix 3.1: Proof of Lemma 3.1}

We first define another convenient equivalence relation.

Definition 13.1 Given $\omega_{1}$ and $\omega_{2}$ two connected components of $\omega$, we say that $\omega_{1} \bumpeq$ $\omega_{2}$ if there are $N \in \mathbb{N}$ and $N$ connected components $\left(\omega^{i}\right)_{1 \leq i \leq N}$ of $\omega$ such that 
- we have $\omega_{1} \mathcal{R}_{\phi} \omega^{(1)}$,

- for all $1 \leq i \leq N-1$, we have $\omega^{(i)} \mathcal{R}_{\phi} \omega^{(i+1)}$,

- we have $\omega^{(N)} \mathcal{R}_{\phi} \omega_{2}$.

The relation $\bumpeq$ is an equivalence relation on the set of connected components of $\omega$. For $\omega_{1}$ a connected component of $\omega$, we denote its equivalence class for $\bumpeq$ by $\left\{\omega_{1}\right\}$.

The following result is useful for the proof of Lemma 3.1.

Lemma 13.2 Let $\Omega_{0}$ be a connected component of $\bigcup_{s \in \mathbb{R}^{+}} \phi_{-s}(\omega)$ and let $\left(\omega_{\ell}\right)_{\ell \in L}$ be the connected components of $\omega$ such that for all $\ell \in L$, there exists $t \geq 0$ with $\phi_{-t}\left(\omega_{\ell}\right) \cap \Omega_{0} \neq \emptyset$. Then, for all $\ell, \ell^{\prime} \in L$, we have $\omega_{\ell} \bumpeq \omega_{\ell^{\prime}}$.

Proof of Lemma 13.2 Assume that there exist at least two equivalence classes for $\bumpeq$ among the $\omega_{\ell}, \ell \in L$. Let $\ell_{0} \in L$ and consider $\left\{\omega_{\ell_{0}}\right\}$ the equivalence class of $\omega_{\ell_{0}}$ for $\bumpeq$. Defining

$$
\begin{gathered}
U_{1}:=\bigcup_{U \in \mathcal{C C}(\omega), U \in\left\{\omega_{\ell_{0}}\right\}} \bigcup_{t \geq 0} \phi_{-t}(U) \cap \Omega_{0} \quad \text { and } \\
U_{2}:=\bigcup_{U \in \mathcal{C C C}(\omega), U \notin\left\{\omega_{\ell_{0}}\right\}} \bigcup_{t \geq 0} \phi_{-t}(U) \cap \Omega_{0},
\end{gathered}
$$

we have by construction that $U_{1}, U_{2}$ are two open non-empty subsets of $\Omega_{0}$ and that $U_{1} \cup U_{2}=\Omega_{0}$.

Let us check $U_{1} \cap U_{2}=\emptyset$ : otherwise it means that there exist two connected components $\Omega_{1}$ and $\Omega_{2}$ of $\omega$ such that $\Omega_{1} \in\left\{\omega_{\ell_{0}}\right\}, \Omega_{2} \notin\left\{\omega_{\ell_{0}}\right\}$ and $\Omega_{1} \mathcal{R}_{\phi} \Omega_{2}$, which is excluded by definition of the equivalence class.

This is a contradiction with the fact that $\Omega_{0}$ is connected.

We are now in position to prove Lemma 3.1.

Proof of Lemma 3.1 Let us first prove that $\omega_{1} \approx \omega_{2} \Longrightarrow \Psi\left(\omega_{1}\right) \sim \Psi\left(\omega_{2}\right)$. It suffices to prove that

$$
\left(\omega_{1} \mathcal{R}_{k} \omega_{2} \text { or } \omega_{1} \mathcal{R}_{\phi} \omega_{2}\right) \Longrightarrow\left(\Psi\left(\omega_{1}\right) \mathcal{R}_{k} \Psi\left(\omega_{2}\right) \text { or } \Psi\left(\omega_{1}\right) \mathcal{R}_{\phi} \Psi\left(\omega_{2}\right)\right)
$$

The conclusion then follows from an iterative use of this argument.

If $\omega_{1} \mathcal{R}_{k} \omega_{2}$, then $\Psi\left(\omega_{1}\right) \mathcal{R}_{k} \Psi\left(\omega_{2}\right)$. This follows from the fact that $\omega_{j} \subset \Psi\left(\omega_{j}\right)$ and the definition of $\mathcal{R}_{k}$. Similarly, if $\omega_{1} \mathcal{R}_{\phi} \omega_{2}$, then $\Psi\left(\omega_{1}\right) \mathcal{R}_{\phi} \Psi\left(\omega_{2}\right)$.

Let us now prove that $\Psi\left(\omega_{1}\right) \sim \Psi\left(\omega_{2}\right) \Longrightarrow \omega_{1} \approx \omega_{2}$. According to Lemma 13.2, it is sufficient to prove that $\Omega^{(1)}, \Omega^{(2)}$ being two given connected components of $\bigcup_{t \geq 0} \phi_{-t}(\omega)$, we have

$$
\Omega^{(1)} \mathcal{R}_{k} \Omega^{(2)} \Longrightarrow \begin{aligned}
& \text { there exist two connected components } \omega_{1}^{*} \text { and } \omega_{2}^{*} \text { of } \omega \\
& \text { such that } \omega_{1}^{*} \mathcal{R}_{k} \omega_{2}^{*} \text { and } \omega_{1}^{*} \subset \Omega^{(1)}, \omega_{2}^{*} \subset \Omega^{(2)}
\end{aligned}
$$

The conclusion then follows from an iterative use of this argument. 
By definition of $\mathcal{R}_{k}$, there exist $\left(x, v_{1}, v_{2}\right) \in \mathbb{T}^{d} \times \mathbb{R}^{d} \times \mathbb{R}^{d}$ with $\left(x, v_{1}\right) \in \Omega^{(1)}$ and $\left(x, v_{2}\right) \in \Omega^{(2)}$ such that $k\left(x, v_{1}, v_{2}\right)>0$ or $k\left(x, v_{2}, v_{1}\right)>0$.

Note in particular that this implies $\left(x, v_{1}\right),\left(x, v_{2}\right) \in \omega$. Denoting by $\omega_{1}^{*}$ (resp. $\left.\omega_{2}^{*}\right)$ the connected component of $\omega$ such that $\left(x, v_{1}\right) \in \omega_{1}^{*}\left(\operatorname{resp} .\left(x, v_{2}\right) \in \omega_{2}^{*}\right)$, we hence have $\omega_{1}^{*} \mathcal{R}_{k} \omega_{2}^{*}$. The conclusion of (13.2) follows from the fact that $\omega_{j}^{*} \subset \Omega^{(j)}$, for $j=1,2$ and the very definition of $\mathcal{R}_{k}$.

\section{Appendix 3.2: Almost Everywhere Geometric Control Conditions and Connectedness}

In this section, we give sufficient conditions for $\omega$ to satisfy the a.e.i.t. GCC with $\bigcup_{s \in \mathbb{R}^{+}} \phi_{-s}(\omega)$ connected. In this case, whatever the collision kernel $k$, there is a single equivlence class for $\approx$ and $\sim$, so that the dimension of the set of stationary solutions is one (and the equilibrium ultimately reached is the global Maxwellian).

Proposition 13.1 Let $\omega \subset \mathbb{T}^{d} \times \mathbb{R}^{d}$ be an open subset. Consider the following geometric properties:

(i) There exists $\tilde{\omega} \subset \omega, \tilde{\omega}$ connected satisfying the a.e.i.t. Geometric Control Condition;

(ii) The set $\omega$ satisfies the a.e.i.t. GCC and for any connected components $\left(\omega_{1}, \omega_{2}\right)$ of $\omega$, there exists $\left(x_{0}, v_{0}\right) \in \omega_{1}$ and $s \in \mathbb{R}$ such that $\phi_{s}\left(x_{0}, v_{0}\right) \in \omega_{2}$;

(iii) The set $\omega$ satisfies the a.e.i.t. GCC and $\bigcup_{s \in \mathbb{R}^{+}} \phi_{-s}(\omega)$ is connected.

Then $(i) \Longrightarrow$ (iii) and (ii) $\Longrightarrow$ (iii).

Note in particular that $(i)-(i i)-(i i i)$ hold as soon as $\omega$ is connected and satisfies a.e.i.t. GCC.

Proof of Proposition 13.1 Before starting the proof, let us remark that is $\omega$ is a connected open subset of $\Omega \times \mathbb{R}^{d}$, then $\bigcup_{s \in \mathbb{R}^{+}} \phi_{-s}(\omega)$ is also a connected open subset. Indeed it is first an open subset of $\Omega \times \mathbb{R}^{d}$, and it is equivalent to show that it is path-connected. Let $y_{1}, y_{2} \in \bigcup_{s \in \mathbb{R}^{+}} \phi_{-s}(\omega)$; there exists $s_{1}, s_{2} \geq 0$ and $z_{1}, z_{2} \in \omega$ such that $y_{1}=\phi_{-s_{1}}\left(z_{1}\right)$ and $y_{2}=\phi_{-s_{2}}\left(z_{2}\right)$. Since $\omega$ is a connected open subset of $\Omega \times \mathbb{R}^{d}$, it is also path-connected and one can find a continuous path in $\omega$ between $z_{1}$ and $z_{2}$. Using the application $\phi_{-s}$, we also get a continuous path between $y_{1}$ and $z_{1}$ in $\bigcup_{s \in \mathbb{R}^{+}} \phi_{-s}(\omega)$ (resp. between $y_{2}$ and $z_{2}$ ).

Gluing these paths together, this yields a continuous path in $\bigcup_{s \in \mathbb{R}^{+}} \phi_{-s}(\omega)$ between $y_{1}$ and $y_{2}$.

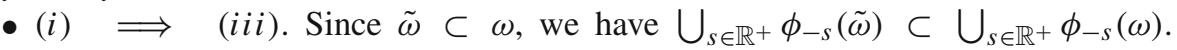
Denote by $\left(\Omega_{i}\right)_{i \in I}$ the connected components of $\bigcup_{s \in \mathbb{R}^{+}} \phi_{-s}(\omega)$. The sets $\Omega_{i}$ are connected open sets so that the inclusion $\bigcup_{s \in \mathbb{R}^{+}} \phi_{-s}(\tilde{\omega}) \subset \bigcup_{s \in \mathbb{R}^{+}} \phi_{-s}(\omega)$ together with the connectedness of $\bigcup_{s \in \mathbb{R}^{+}} \phi_{-s}(\tilde{\omega})$ yields the existence of $i_{0} \in I$ such that $\bigcup_{s \in \mathbb{R}^{+}} \phi_{-s}(\tilde{\omega}) \subset \Omega_{i_{0}}$. Since $\bigcup_{s \in \mathbb{R}^{+}} \phi_{-s}(\tilde{\omega})$ is of full measure, this is also the case for $\Omega_{i_{0}}$. As $\Omega_{i}$ is open, we obtain that $\Omega_{i}=\emptyset$ for $i \neq i_{0}$, so that $\Omega_{i_{0}}=\bigcup_{s \in \mathbb{R}^{+}} \phi_{-s}(\omega)$ is connected (and of full measure).

- (ii $) \Longrightarrow($ iii $)$. Let $y_{1}, y_{2} \in \bigcup_{s \in \mathbb{R}^{+}} \phi_{-s}(\omega)$; there exists $s_{1}, s_{2} \geq 0$ and $z_{1}, z_{2} \in \omega$ such that $y_{1}=\phi_{-s_{1}}\left(z_{1}\right)$ and $y_{2}=\phi_{-s_{2}}\left(z_{2}\right)$. If $z_{1}, z_{2}$ belong to the same connected 
component $\tilde{\omega}$ of $\omega$, then since $\bigcup_{s \in \mathbb{R}^{+}} \phi_{-s}(\tilde{\omega})$ is connected, one can find a continuous path between $z_{1}$ and $z_{2}$.

If $z_{1}, z_{2}$ belong to two different connected components $\omega_{1}$ and $\omega_{2}$, apply $(i)$ to find, up to a permutation between the indices 1 and $2, u \in \omega_{1}$ and $s \in \mathbb{R}^{+}$such that $\phi_{-s}(u) \in \omega_{2}$. Then one can find a continuous path between $u$ and $z_{1}$ in $\omega_{1}$, and another between $\phi_{-s}(u)$ and $z_{2}$ in $\omega_{2}$. We conclude as in the previous subcase by gluing the paths together.

\section{Appendix 4: Proof of Proposition 3.2}

In this section, we prove Proposition 3.2.

Proof of Proposition 3.2 Let $x_{0} \in \mathbb{T}^{d} \backslash \overline{p_{x}(\omega)} \neq \varnothing$ and take $\eta>0$ such that $B\left(x_{0}, 2 \eta\right) \cap \overline{p_{x}(\omega)}=\emptyset$. Define the potential $V(x):=\frac{\left|x-x_{0}\right|^{2}}{2} \Psi(x)$, where $\Psi$ is a "corrector" to ensure $V \in C^{\infty}\left(\mathbb{T}^{d}\right)$, and such that $\Psi \equiv 1$ on $B\left(x_{0}, 2 \eta\right)$ (reduce $\eta$ if necessary). Denote $V_{\varepsilon}=\varepsilon V$ and notice that $\nabla V_{\varepsilon}(x)=\varepsilon\left(x-x_{0}\right)$ on $B\left(x_{0}, 2 \eta\right)$. As a consequence, the hamiltonian flow $\left(\phi_{t}\right)_{t \in \mathbb{R}}$ associated to the vector field $v \cdot \nabla_{x}-\nabla_{x} V_{\varepsilon} \cdot \nabla_{v}$ may be explicited in the set $B\left(x_{0}, 2 \eta\right) \times \mathbb{R}^{d}$ : we have

$$
\begin{aligned}
\phi_{t}(x, v)= & \left(x_{0}+\left(x-x_{0}\right) \cos (\sqrt{\varepsilon} t)+\frac{v}{\sqrt{\varepsilon}} \sin (\sqrt{\varepsilon} t),-\left(x-x_{0}\right) \sqrt{\varepsilon} \sin (\sqrt{\varepsilon} t)\right. \\
& +v \cos (\sqrt{\varepsilon} t))
\end{aligned}
$$

as long as $\phi_{t}(x, v) \in B\left(x_{0}, 2 \eta\right) \times \mathbb{R}^{d}$. In particular, note that if $(x, v) \in B\left(x_{0}, \eta\right) \times$ $B(0, \sqrt{\varepsilon} \eta)$, then $\phi_{t}(x, v)$ remains in $B\left(x_{0}, 2 \eta\right) \times B(0,2 \sqrt{\varepsilon} \eta)$ for all $t \in \mathbb{R}^{+}$. This reads $\phi_{t}\left(B\left(x_{0}, \eta\right) \times B(0, \sqrt{\varepsilon} \eta)\right) \subset B\left(x_{0}, 2 \eta\right) \times B(0,2 \sqrt{\varepsilon} \eta)$, i.e. in particular $\phi_{t}\left(B\left(x_{0}, \eta\right) \times\right.$ $B(0, \sqrt{\varepsilon} \eta)) \cap \omega=\emptyset$ for all $t \in \mathbb{R}^{+}$. This proves that a.e.i.t. GCC is not satisfied.

Remark 14.1 Notice that in the previous proof, to handle small potential, we consider small speeds, i.e. $v \in B(0, \sqrt{\varepsilon} \eta)$. In the opposite direction, if one fixes the speeds in a large Hamiltonian sphere $v \in S_{H}(0, R)$ (note that with the particular potential used in the proof, on the set $\{\Psi=1\}$ we have $S_{H}(0, R)=S(0, R)$ ) for some $R>0$, then one can find a (large) potential (namely $R^{2} / \eta^{2} V$ where $V$ is that of the previous proof) such that a.e.i.t. GCC fails.

\section{Appendix 5: Other Linear Boltzmann Type Equations}

The goal of this appendix is to show that the methods developed here can be adapted to handle other types of Boltzmann-like equations. 


\section{Appendix 5.1: Generalization to a Wider Class of Kinetic Transport Equations}

Here, we consider the equation

$$
\partial_{t} f+a(v) \cdot \nabla_{x} f-\nabla_{x} V \cdot \nabla_{v} f=\int_{\mathbb{R}^{d}}\left[k\left(x, v^{\prime}, v\right) f\left(v^{\prime}\right)-k\left(x, v, v^{\prime}\right) f(v)\right] d v^{\prime},
$$

where $a(v)=\nabla_{v} A(v)$ with $A \in W_{l o c}^{2, \infty}\left(\mathbb{R}^{d}\right)$ is such that $A(v) \rightarrow+\infty$ as $|v| \rightarrow+\infty$ and $\int_{\mathbb{R}^{d}} e^{-A(v)} d v<+\infty$.

For simplicity, we assume that (15.1) is set on $\mathbb{T}^{d} \times \mathbb{R}^{d}$.

Assume that $a(v)$ satisfies a non degeneracy property: there exists $\gamma \in(0,2)$ and $C>0$ such that, for all $\xi \in \mathbb{S}^{d-1}$,

$$
\operatorname{Leb}\left\{v \in \mathbb{R}^{d},|a(v) \cdot \xi| \leq \varepsilon\right\} \leq C \varepsilon^{\gamma}
$$

This assumption prevents from concentration of $a(v)$ in some directions of $\mathbb{S}^{d-1}$.

The hamiltonian associated to the transport equation is then the following:

$$
H(x, v)=A(v)+V(x) .
$$

Define the global Maxwellian associated to $a(v)$ by

$$
\mathcal{M}_{A}(v)=C_{A} e^{-A(v)},
$$

with $C_{A}=1 /\left(\int_{\mathbb{R}^{d}} e^{-A(v)} d v\right)$.

In addition to the usual assumption $\mathbf{A} \mathbf{1}$ on the collision kernel $k$, we shall assume the following (which replace $\mathbf{A} \mathbf{2}-\mathbf{A 3}$ ):

A2'. We assume that $\mathcal{M}_{A}$ cancels the collision operator, that is

$$
\text { for all }(x, v) \in \mathbb{T}^{d} \times \mathbb{R}^{d}, \quad \int_{\mathbb{R}^{d}}\left[k\left(x, v^{\prime}, v\right) \mathcal{M}_{A}\left(v^{\prime}\right)-k\left(x, v, v^{\prime}\right) \mathcal{M}_{A}(v)\right] d v^{\prime}=0 \text {. }
$$

A3'. We assume that

$$
\tilde{k}\left(x, v^{\prime}, v\right):=\frac{k\left(x, v^{\prime}, v\right)}{\mathcal{M}_{A}(v)} \in L^{\infty}\left(\mathbb{T}^{d} \times \mathbb{R}^{d} \times \mathbb{R}^{d}\right)
$$

For $a(v)=v$ (for which $\gamma=1$ in (15.2)), we recover the framework which has been already treated before. One physically relevant case is

$$
a_{r e l}(v):=\frac{v}{\sqrt{1+|v|^{2}}}
$$


(for which we also have $\gamma=1$ in (15.2)), which allows to model relativistic transport. Note that in this case, we have $A_{r e l}(v):=\sqrt{1+|v|^{2}}$, and the related Maxwellian is then the so-called relativistic Maxwellian:

$$
\mathcal{M}_{\text {rel }}(v):=C_{r e l} e^{-\sqrt{1+|v|^{2}}}
$$

where $C_{r e l}$ is a normalizing constant, so that $\int_{\mathbb{R}^{d}} \mathcal{M}_{\text {rel }}(v) d v=1$.

Our aim in this paragraph is to show that our methods are still relevant. The characteristics of the equation are defined in the following way:

Definition 15.1 Let $V \in W_{l o c}^{2, \infty}\left(\mathbb{T}^{d}\right)$. Let $\left(x_{0}, v_{0}\right) \in \mathbb{T}^{d} \times \mathbb{R}^{d}$. The characteristics $\phi_{t}\left(x_{0}, v_{0}\right):=\left(X_{t}\left(x_{0}, v_{0}\right), \Xi_{t}\left(x_{0}, v_{0}\right)\right)$ associated to the hamiltonian $H(x, v)=$ $A(v)+V(x)$ are defined as the solutions to the system:

$$
\left\{\begin{array}{l}
\frac{d X_{t}}{d t}=a\left(\Xi_{t}\right), \quad \frac{d \Xi_{t}}{d t}=-\nabla_{x} V\left(X_{t}\right), \\
X_{t=0}=x_{0}, \quad \Xi_{t=0}=v_{0} .
\end{array}\right.
$$

With this definition of characteristics, we can then properly define

- the set $\omega$ where collisions are effective, as in Definition 2.1,

- the Unique Continuation Property, as in Definition 2.11

- $C^{-}(\infty)$, as in Definition 2.4,

- a.e.i.t. GCC, as in Definition 2.5,

- the equivalence relation $\sim$, as in Definition 3.1.

The next thing to do concerns the local well-posedness of (15.1) in some relevant weighted spaces, which we introduce below.

Definition 15.2 (Weighted $L^{p}$ spaces). We define the Banach spaces $\mathcal{L}_{A}^{2}$ and $\mathcal{L}_{A}^{\infty}$ by

$$
\begin{aligned}
& \mathcal{L}_{A}^{2}:=\left\{f \in L_{l o c}^{1}\left(\mathbb{T}^{d} \times \mathbb{R}^{d}\right), \int_{\mathbb{T}^{d} \times \mathbb{R}^{d}}|f|^{2} \frac{e^{V}}{\mathcal{M}_{A}(v)} d v d x<+\infty\right\} \\
&\|f\|_{\mathcal{L}_{A}^{2}}=\left(\int_{\mathbb{T}^{d} \times \mathbb{R}^{d}}|f|^{2} \frac{e^{V}}{\mathcal{M}_{A}(v)} d v d x\right)^{1 / 2}, \\
& \mathcal{L}_{A}^{\infty}:=\left\{f \in L_{l o c}^{1}\left(\mathbb{T}^{d} \times \mathbb{R}^{d}\right), \sup _{\mathbb{T}^{d} \times \mathbb{R}^{d}}|f| \frac{e^{V}}{\mathcal{M}_{A}(v)}<+\infty\right\} \\
&\|f\|_{\mathcal{L}_{A}^{\infty}}=\sup _{\mathbb{T}^{d} \times \mathbb{R}^{d}}|f| \frac{e^{V}}{\mathcal{M}_{A}(v)} .
\end{aligned}
$$

The space $\mathcal{L}_{A}^{2}$ is a Hilbert space endowed with the inner product

$$
\langle f, g\rangle_{\mathcal{L}_{A}^{2}}:=\int_{\mathbb{T}^{d} \times \mathbb{R}^{d}} e^{V} \frac{f g}{\mathcal{M}_{A}(v)} d v d x .
$$

As usual, we have 
Proposition 15.3 (Well-posedness of the linear Boltzmann equation with modified transport). Assume that $f_{0} \in \mathcal{L}_{A}^{2}$. Then there exists a unique $f \in C^{0}\left(\mathbb{R} ; \mathcal{L}_{A}^{2}\right)$ solution of (15.1) satisfying $\left.f\right|_{t=0}=f_{0}$, and we have

$$
\text { for all } t \geq 0, \quad \frac{d}{d t}\|f(t)\|_{\mathcal{L}_{A}^{2}}^{2}=-\mathscr{D}_{A}(f(t))
$$

where

$$
\begin{aligned}
\mathscr{D}_{A}(f)=\frac{1}{2} & \int_{\Omega} e^{V} \int_{\mathbb{R}^{d}} \int_{\mathbb{R}^{d}}\left(\frac{k\left(x, v^{\prime}, v\right)}{\mathcal{M}_{A}(v)}+\frac{k\left(x, v, v^{\prime}\right)}{\mathcal{M}_{A}\left(v^{\prime}\right)}\right) \mathcal{M}_{A}(v) \mathcal{M}_{A}\left(v^{\prime}\right) \\
& \times\left(\frac{f(v)}{\mathcal{M}_{A}(v)}-\frac{f\left(v^{\prime}\right)}{\mathcal{M}_{A}\left(v^{\prime}\right)}\right)^{2} d v^{\prime} d v d x
\end{aligned}
$$

If moreover $f_{0} \geq 0$ a.e., then for all $t \in \mathbb{R}$ we have $f(t, \cdot, \cdot) \geq 0$ a.e. (Maximum principle).

Then the analogues of Theorems 2.1, 2.2 and 2.3 hold in this setting (with some obvious modifications); for the sake of conciseness, we omit these statements.

Such results can be proved exactly as Theorems 2.1, 2.2 and 2.3. The crucial additional ingredient is the fact that averaging lemmas for the operator $a(v) \cdot \nabla_{x}$ still hold, precisely when $a$ satisfies the non degeneracy condition (15.2), see [23]. Note that in this case, the gain of regularity on averages depends on the index $\gamma$ in (15.2), but in any case, this is always sufficient to obtain compactness.

\section{Appendix 5.2: Generalization to linearized BGK operators}

Once again, for simplicity, we assume that $(x, v) \in \mathbb{T}^{d} \times \mathbb{R}^{d}$. Let $V \in W_{l o c}^{2, \infty}\left(\mathbb{T}^{d}\right)$. Let $\varphi: \mathbb{R} \rightarrow \mathbb{R}_{*}^{+}$be a function in $L^{\infty}(\mathbb{R})$ such that

$$
\int_{\mathbb{T}^{d} \times \mathbb{R}^{d}} \varphi\left(\frac{|v|^{2}}{2}+V(x)\right) d v d x<+\infty
$$

Denote $F(x, v)=\varphi\left(\frac{|v|^{2}}{2}+V(x)\right)$ and $\rho_{F}(x)=\int F(x, v) d v$.

Let $\sigma \in C^{0}\left(\mathbb{T}^{d}\right)$ be a non-negative function. We study in this paragraph the following degenerate linearized BGK equation:

$$
\partial_{t} f+v \cdot \nabla_{x} f-\nabla_{x} V \cdot \nabla_{v} f=\sigma(x)\left(\frac{\int_{\mathbb{R}^{d}} f d v}{\rho_{F}(x)} F(x, v)-f\right) .
$$

with an initial condition $f_{0}$ at time 0 . The natural equilibrium is given by

$$
(x, v) \mapsto \int_{\mathbb{T}^{d} \times \mathbb{R}^{d}} f_{0} d v d x \frac{F(x, v)}{\int_{\mathbb{T}^{d} \times \mathbb{R}^{d}} F(x, v) d v d x} .
$$


The main feature of this equilibrium is that there is no separation of variables contrary to the Maxwellian case.

Our aim in this paragraph is again to show that the methods developed in this paper are still relevant here. For what concerns well-posedness, we introduce the relevant weighted $L^{p}$ spaces and have the usual result.

Definition 15.4 (Weighted $L^{p}$ spaces). We define the Banach spaces $\mathcal{L}_{b g k}^{2}$ and $\mathcal{L}_{b g k}^{\infty}$ by

$$
\begin{aligned}
\mathcal{L}_{b g k}^{2} & :=\left\{f \in L_{l o c}^{1}\left(\mathbb{T}^{d} \times \mathbb{R}^{d}\right), \int_{\mathbb{T}^{d} \times \mathbb{R}^{d}}|f|^{2} \frac{1}{F(x, v)} d v d x<+\infty\right\}, \\
\|f\|_{\mathcal{L}_{b g k}^{2}} & =\left(\int_{\mathbb{T}^{d} \times \mathbb{R}^{d}}|f|^{2} \frac{1}{F(x, v)} d v d x\right)^{1 / 2}, \\
\mathcal{L}_{b g k}^{\infty} & :=\left\{f \in L_{l o c}^{1}\left(\mathbb{T}^{d} \times \mathbb{R}^{d}\right), \sup _{\mathbb{T}^{d} \times \mathbb{R}^{d}}|f| \frac{1}{F(x, v)}<+\infty\right\}, \\
\|f\|_{\mathcal{L}^{\infty}} & =\sup _{\mathbb{T}^{d} \times \mathbb{R}^{d}}|f| \frac{1}{F(x, v)}
\end{aligned}
$$

The space $\mathcal{L}_{b g k}^{2}$ is a Hilbert space endowed with the inner product

$$
\langle f, g\rangle_{\mathcal{L}_{b g k}^{2}}:=\int_{\mathbb{T}^{d} \times \mathbb{R}^{d}} \frac{f g}{F(x, v)} d v d x
$$

Proposition 15.5 (Well-posedness of the linearized BGK equation). Assume that $f_{0} \in$ $\mathcal{L}_{b g k}^{2}$. Then there exists a unique $f \in C^{0}\left(\mathbb{R} ; \mathcal{L}_{b g k}^{2}\right)$ solution of (15.11) satisfying $\left.f\right|_{t=0}=f_{0}$, and we have

$$
\text { for all } t \geq 0, \quad \frac{d}{d t}\|f(t)\|_{\mathcal{L}_{b g k}^{2}}^{2}=-\mathscr{D}_{b g k}(f(t))
$$

where

$$
\mathscr{D}_{b g k}(f)=\int_{\mathbb{T}^{d}} e^{V} \sigma(x) \int_{\mathbb{R}^{d}} \int_{\mathbb{R}^{d}} \frac{F(x, v) F\left(x, v^{\prime}\right)}{\rho_{F}(x)}\left(\frac{f(v)}{F(x, v)}-\frac{f\left(v^{\prime}\right)}{F\left(x, v^{\prime}\right)}\right)^{2} d v^{\prime} d v d x .
$$

If moreover $f_{0} \geq 0$ a.e., then for all $t \in \mathbb{R}$ we have $f(t, \cdot, \cdot) \geq 0$ a.e. (Maximum principle).

With the same geometric definitions of Section 2, we have the following results. Note that the set $\omega$ where the collisions are effective is equal to $\omega_{x} \times \mathbb{R}^{d}$, where

$$
\omega_{x}:=\left\{x \in \mathbb{T}^{d}, \sigma(x)>0\right\}
$$

Theorem 15.6 (Convergence to equilibrium). The following statements are equivalent. 
(i.) The set $\omega$ satisfies the Unique Continuation Property.

(ii.) The set $\omega$ satisfies the a.e.i.t. GCC and $\bigcup_{s \in \mathbb{R}^{+}} \phi_{-s}(\omega)$ is connected.

(iii.) For all $f_{0} \in \mathcal{L}_{b g k}^{2}$, denote by $f(t)$ the unique solution to (15.11) with initial datum $f_{0}$. We have

$$
\left\|f(t)-\left(\int_{\mathbb{T}^{d} \times \mathbb{R}^{d}} f_{0} d v d x\right) \frac{F(x, v)}{\int_{\mathbb{T}^{d} \times \mathbb{R}^{d}} F(x, v) d v d x}\right\|_{\mathcal{L}_{b g k}^{2}} \rightarrow_{t \rightarrow+\infty} 0,
$$

Theorem 15.7 (Exponential convergence to equilibrium). The two following statements are equivalent:

(i.) $C^{-}(\infty)>0$.

(ii.) There exists $C>0, \gamma>0$ such that for any $f_{0} \in \mathcal{L}^{2}\left(\mathbb{T}^{d} \times \mathbb{R}^{d}\right)$, the unique solution to (15.11) with initial datum $f_{0}$ satisfies

$$
\begin{aligned}
& \left\|f(t)-\left(\int_{\mathbb{T}^{d} \times \mathbb{R}^{d}} f_{0} d v d x\right) \frac{F(x, v)}{\int_{\mathbb{T}^{d} \times \mathbb{R}^{d}} F(x, v) d v d x}\right\|_{\mathcal{L}^{2}} \\
& \leq C e^{-\gamma t}\left\|f_{0}-\left(\int_{\mathbb{T}^{d} \times \mathbb{R}^{d}} f_{0} d v d x\right) \frac{F(x, v)}{\int_{\mathbb{T}^{d} \times \mathbb{R}^{d}} F(x, v) d v d x}\right\|_{\mathcal{L}^{2}} .
\end{aligned}
$$

We shall not dwell on the proofs of Theorems 15.6 and 15.7, since they are very similar to those of Theorems 2.2 and 2.3. Indeed, we note that the structure of the equation (15.11) is similar to that of (1.1), in the sense that the "degenerate dissipative" part is still made of a dissipative term plus a relatively compact term. This compactness, as usual, comes from averaging lemmas.

Let us just underline a crucial point in the proof (ii.) implies $(i$.) of Theorem 15.6. Here, we have to be careful of the fact that $F(x, v)$ does not separate the $x$ and $v$ variables, contrary to the Maxwellian equilibrium of (1.1). Let us check that the proof we gave in the Boltzmann case is still relevant (see the proof of (ii.) $\Longrightarrow \quad$ (i.) of Theorem 2.2).

Let $f \in C_{t}^{0}\left(\mathcal{L}_{b g k}^{2}\right)$ be a solution to

$$
\begin{gathered}
\partial_{t} f+v \cdot \nabla_{x} f-\nabla_{x} V \cdot \nabla_{v} f=0, \\
f=\rho(t, x) F(x, v) \text { on } \mathbb{R}^{+} \times \omega .
\end{gathered}
$$

Assume that $\int_{\mathbb{T}^{d} \times \mathbb{R}^{d}} f d v d x=0$. The goal is to show that $f=0$.

To this purpose, as before, consider for $(t, x, v) \in \mathbb{R}^{+} \times \omega, g(t, x):=\frac{1}{F(x, v)} f$ (note that by (15.16), $g$ does not depend on $v$ ). We have, for $(t, x, v) \in \mathbb{R}^{+} \times \omega$ :

$$
\partial_{t} g+v \cdot \nabla_{x} g=\frac{1}{F(x, v)}\left[\partial_{t} f+v \cdot \nabla_{x} f-v \cdot \nabla_{x} F \frac{f}{F}\right] .
$$


Since $f$ satisfies (15.15) and (15.16),

$$
\partial_{t} f+v \cdot \nabla_{x} f=\nabla_{x} V \cdot \nabla_{v} f=\nabla_{v} F \cdot \nabla_{x} V \frac{f}{F} .
$$

By definition of $F$, we have

$$
\nabla_{x} V \cdot \nabla_{v} F=v \cdot \nabla_{x} F
$$

from which we deduce that $g$ satisfies the free transport equation on $\omega$ :

$$
\text { for all }(t, x, v) \in \mathbb{R}^{+} \times \omega, \quad \partial_{t} g+v \cdot \nabla_{x} g=0 \text {. }
$$

We then conclude as in the proof of $($ ii. $) \Longrightarrow($ i.) of Theorem 2.2, mutatis mutandis.

\section{References}

1. Agoshkov, V.I.: Spaces of functions with differential-difference characteristics and the smoothness of solutions of the transport equation. Dokl. Akad. Nauk SSSR 276(6), 1289-1293 (1984)

2. Arsénio, D., Saint-Raymond, L.: Compactness in kinetic transport equations and hypoellipticity. J. Funct. Anal. 261(10), 3044-3098 (2011)

3. Bardos, C., Lebeau, G., Rauch, J.: Sharp sufficient conditions for the observation, control, and stabilization of waves from the boundary. SIAM J. Control Optim. 30, 1024-1065 (1992)

4. Bernard, É., Salvarani, F.: On the convergence to equilibrium for degenerate transport problems. Arch. Ration. Mech. Anal. 208(3), 977-984 (2013)

5. Bernard, É., Salvarani, F.: On the exponential decay to equilibrium of the degenerate linear Boltzmann equation. J. Funct. Anal. 265(9), 1934-1954 (2013)

6. Bernard, É., Salvarani, F.: Optimal estimate of the spectral gap for the degenerate Goldstein-Taylor model. J. Stat. Phys. 153(2), 363-375 (2013)

7. Berthelin, F., Junca, S.: Averaging lemmas with a force term in the transport equation. J. Math. Pures Appl. (9) 93(2), 113-131 (2010)

8. Bézard, M.: Régularité $L^{p}$ précisée des moyennes dans les équations de transport. Bull. Soc. Math. France 122(1), 29-76 (1994)

9. Bodineau, T., Gallagher, I., Saint-Raymond, L.: The Brownian motion as the limit of a deterministic system of hard-spheres. arXiv preprint arXiv:1305.3397 (2013)

10. Cáceres, M.J., Carrillo, J.A., Goudon, T.: Equilibration rate for the linear inhomogeneous relaxationtime Boltzmann equation for charged particles. Commun. Part. Differ. Equ. 28(5-6), 969-989 (2003)

11. Cercignani, C.: The Boltzmann Equation and Its Applications. Applied Mathematical Sciences, vol. 67. Springer-Verlag, New York (1988)

12. Dautray, R., Lions, J.-L.: Mathematical Analysis and Numerical Methods for Science and Technology, vol. 1. Springer-Verlag, Berlin (1990). Physical origins and classical methods, With the collaboration of Philippe Bénilan, Michel Cessenat, André Gervat, Alain Kavenoky and Hélène Lanchon, Translated from the French by Ian N. Sneddon, With a preface by Jean Teillac

13. Dautray, R., Lions, J.-L.: Mathematical Analysis and Numerical Methods for Science and Technology, vol. 6. Springer-Verlag, Berlin (1993). Evolution problems. II, With the collaboration of Claude Bardos, Michel Cessenat, Alain Kavenoky, Patrick Lascaux, Bertrand Mercier, Olivier Pironneau, Bruno Scheurer and Rémi Sentis, Translated from the French by Alan Craig

14. De Vuyst, F., Salvarani, F.: Numerical simulations of degenerate transport problems. Kinet. Relat. Models. 7(3), 463-476 (2014)

15. Degond, P., Goudon, T., Poupaud, F.: Diffusion limit for nonhomogeneous and non-micro-reversible processes. Indiana Univ. Math. J. 49(3), 1175-1198 (2000)

16. Desvillettes, L., Salvarani, F.: Asymptotic behavior of degenerate linear transport equations. Bull. Sci. Math. 133(8), 848-858 (2009) 
17. Desvillettes, L., Villani, C.: On the trend to global equilibrium in spatially inhomogeneous entropydissipating systems: the linear Fokker-Planck equation. Commun. Pure Appl. Math. 54(1), 1-42 (2001)

18. DiPerna, R.J., Lions, P.-L.: Global weak solutions of Vlasov-Maxwell systems. Commun. Pure Appl. Math. 42(6), 729-757 (1989)

19. DiPerna, R.J., Lions, P.-L., Meyer, Y.: $L^{p}$ regularity of velocity averages. Ann. Inst. H. Poincaré Anal. Non Linéaire 8(3-4), 271-287 (1991)

20. Dolbeault, J., Mouhot, C., Schmeiser, C.: Hypocoercivity for linear kinetic equations conserving mass. arXiv preprint arXiv:1005.1495, to appear in Trans. AMS (2010)

21. Erdős, L., Yau, H.-T.: Linear Boltzmann equation as the weak coupling limit of a random Schrödinger equation. Commun. Pure Appl. Math. 53(6), 667-735 (2000)

22. Gérard, P., Golse, F.: Averaging regularity results for PDEs under transversality assumptions. Commun. Pure Appl. Math. 45(1), 1-26 (1992)

23. Golse, F., Lions, P.-L., Perthame, B., Sentis, R.: Regularity of the moments of the solution of a transport equation. J. Funct. Anal. 76(1), 110-125 (1988)

24. Golse, F., Perthame, B., Sentis, R.: Un résultat de compacité pour les équations de transport et application au calcul de la limite de la valeur propre principale d'un opérateur de transport. C. R. Acad. Sci. Paris Sér. I Math. 301(7), 341-344 (1985)

25. Han-Kwan, D., Léautaud, M.: Trend to equilibrium and spectral localization properties for the linear Boltzmann equation. Séminaire Laurent S chwartz-Équations aux dérivées partielles et applications. Année 2013-2014. Sémin. Équ. Dériv. Partielles, VII, 15 École Polytech., Palaiseau (2014)

26. Han-Kwan, D., Léautaud, M.: Geometric analysis of the linear Boltzmann equation III. Spectral localization properties. In preparation

27. Han-Kwan, D., Léautaud, M.: Geometric analysis of the linear Boltzmann equation II. Trend to equilibrium in bounded domains with specular conditions. In preparation

28. Hérau, F.: Hypocoercivity and exponential time decay for the linear inhomogeneous relaxation Boltzmann equation. Asymptot. Anal. 46(3-4), 349-359 (2006)

29. Hörmander, L.: The Analysis of Linear Partial Differential Operators. III. Grundlehren der Mathematischen Wissenschaften [Fundamental Principles of Mathematical Sciences], vol. 274. Springer-Verlag, Berlin (1985). Pseudodifferential operators

30. Jabin, P.-E.: Averaging lemmas and dispersion estimates for kinetic equations. Riv. Mat. Univ. Parma 8(1), 71-138 (2009)

31. Koch, H., Tataru, D.: On the spectrum of hyperbolic semigroups. Commun. Part. Differ. Equ. 20(5-6), 901-937 (1995)

32. Lebeau, G.: Équation des ondes amorties. In: de Monvel, AB., Marchenko, V. (eds.) Algebraic and Geometric Methods in Mathematical Physics (Kaciveli, 1993). Mathematical Physics Studies, vol. 19, pp. 73-109. Kluwer Acad. Publ., Dordrecht (1996)

33. Mokhtar-Kharroubi, M.: Optimal spectral theory of the linear Boltzmann equation. J. Funct. Anal. 226(1), 21-47 (2005)

34. Mokhtar-Kharroubi, M.: On $L^{1}$ exponential trend to equilibrium for conservative linear kinetic equations on the torus. J. Funct. Anal. 266(11), 6418-6455 (2014)

35. Mouhot, C., Neumann, L.: Quantitative perturbative study of convergence to equilibrium for collisional kinetic models in the torus. Nonlinearity 19(4), 969-998 (2006)

36. Pazy, A.: Semigroups of Linear Operators and Applications to Partial Differential Equations. Applied Mathematical Sciences, vol. 44. Springer-Verlag, New York (1983)

37. Rauch, J., Taylor, M.: Exponential decay of solutions to hyperbolic equations in bounded domains. Indiana Univ. Math. J. 24, 79-86 (1974)

38. Ukai, S.: On the existence of global solutions of mixed problem for non-linear Boltzmann equation. Proc. Jpn. Acad. 50, 179-184 (1974)

39. Ukai, S., Point, N., Ghidouche, H.: Sur la solution globale du problème mixte de l'équation de Boltzmann nonlinéaire. J. Math. Pures Appl. 57(3), 203-229 (1978)

40. Vidav, I.: Existence and uniqueness of nonnegative eigenfunctions of the Boltzmann operator. J. Math. Anal. Appl. 22, 144-155 (1968)

41. Vidav, I.: Spectra of perturbed semigroups with applications to transport theory. J. Math. Anal. Appl. 30, 264-279 (1970)

42. Villani, C.: Hypocoercivity. Mem. Am. Math. Soc 202(95), iv+141 (2009) 